\title{
Lossless compression and neuron structure extraction for fluorescence microscopy confocal neuron images
}

\author{
Yong Zhang \\ West Virginia University
}

Follow this and additional works at: https://researchrepository.wvu.edu/etd

\section{Recommended Citation}

Zhang, Yong, "Lossless compression and neuron structure extraction for fluorescence microscopy confocal neuron images" (2006). Graduate Theses, Dissertations, and Problem Reports. 2443.

https://researchrepository.wvu.edu/etd/2443

This Dissertation is protected by copyright and/or related rights. It has been brought to you by the The Research Repository @ WVU with permission from the rights-holder(s). You are free to use this Dissertation in any way that is permitted by the copyright and related rights legislation that applies to your use. For other uses you must obtain permission from the rights-holder(s) directly, unless additional rights are indicated by a Creative Commons license in the record and/ or on the work itself. This Dissertation has been accepted for inclusion in WVU Graduate Theses, Dissertations, and Problem Reports collection by an authorized administrator of The Research Repository @ WVU.

For more information, please contact researchrepository@mail.wvu.edu. 
Lossless Compression and Neuron Structure Extraction for Fluorescence Microscopy Confocal Neuron Images

\author{
Yong Zhang
}

Dissertation Submitted to the College of Engineering and Mineral Resources

at West Virginia University

in partial fulfillment of the requirements

for the degree of

\author{
Doctor of Philosophy \\ in \\ Electrical Engineering
}

Donald A. Adjeroh, Ph.D., Chair

V. "Juggy" Jagannathan, Ph.D., Xin Li, Ph.D., Tim McGraw, Ph.D., Steven T.C. Wong, Ph.D.

Xiaobo Zhou, Ph.D.

Lane Department of Computer Science and Electrical Engineering

Morgantown, West Virginia

2006

Keywords: Lossless image compression, PPM, PPAM, Neuron Structure, Dendritic Spine Analysis, Curvilinear Structure Detection.

Copyright 2006 Yong Zhang 


\section{ABSTRACT \\ Lossless Compression and Neuron Structure Extraction for Fluorescence Microscopy Confocal Neuron Images}

\section{Yong Zhang}

To study the development of nervous systems, biologists are interested in neurite outgrowth, differentiation, synapse formation, and plasticity. High throughput neuron image processing is an important method for drug screening and quantitative neurobiological studies. The power of high-throughput processing comes from the automated fluorescence microscopy imaging techniques that make it possible and facile to visualize the complicated biological processes on the cellular and molecular levels and allow fast and cheap acquisition of such imaging data. With this method, a huge number of images are generated, with resolutions at length scales that are small enough to resolve neuron structures. Thus, one immediate challenge facing researchers now is to find efficient and effective methods for managing the unprecedented volume of image data. Accessing these data to generate useful knowledge requires efficient and effective image analysis tools that involve the smallest human interaction. In our work, we study two problems related to neuron images.

The first problem is on lossless compression of neuron images. We consider context based modeling methods, which are seen an important step in high performance lossless data compression. We study methods for effective context modeling for images based on existing successful modeling methods used for text compression. A novel context based modeling method is proposed that is used to compress neuron images in a lossless manner. We also extend the modeling method to compressing other types of images, including natural images. 
The second problem is on neuron structure extraction from neuron images. The neuron structures, including curvilinear neurite segments and dendritic spines, exhibit the connectivity of the neural networks and thus can be used to study the functionality of the neural networks. The extraction and analysis of the neuron structures are still accomplished manually, or semi-automatically. Thus, we are interested in developing fast and fully automatic algorithms for extracting neuron structures. For this purpose, we develop novel methods for extracting curvilinear neurite segments in 2D neuron images and for extracting dendritic spines in 3D neuron images. We also study effective validation methods for evaluating the performance of the proposed neuron structure extraction algorithms. 
To My Wife and My Parents 


\section{ACKNOWLEDGEMENTS}

I would like to express my sincere gratitude to my advisor, Dr. Donald Adjeroh, for his valuable advice throughout my entire graduate study at West Virginia University. Dr. Don was very encouraging, patient, and generous in advising my research work. He was also a very nice person and provided numerous help during my study. I would like to express my sincere gratitude to Dr. Stephen T.C. Wong and Dr. Xiaobo Zhou, for their valuable support and advice throughout my research work at Harvard Medical School. Their foresight, advice, and the excellent research and education environment at Harvard University were very instrumental in my completion of the Ph.D degree.

I would like to thank Dr. Juggy Jagannathan, Dr. Xin Li, and Dr. Tim McGraw at West Virginia University, for serving on my committee and for providing advice, discussion, and comments on my research work. I would like to acknowledge the assistance from our biology collaborators in the Department of Cell Biology, Department of Neurobiology, and Department of Molecular and Cellular Biology, all at Harvard University. Specially, I would like to thank Dr. Junying Yuan, Dr. Alexei Degterev, Dr. Marta Lipinski, Dr. Bernardo Sabatini, Ms. Rochelle Witt, Dr.Jeff Lichtman, Mr.Ju Lu, and Ms. Baillie Yip, for their kind help on my research work.

I also would like to thank all the members at HCNR CBI of Harvard Medical School and the members at the Lane Department of CSEE of WVU for their valuable advice, discussion, and support. 


\section{Table of Contents}

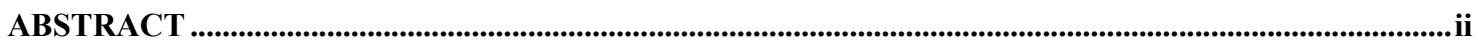

ACKNOWLEDGEMENTS ....................................................................................................................................

LIST OF TABLES ……............................................................................................................................................ ix

LIST OF FIGURES

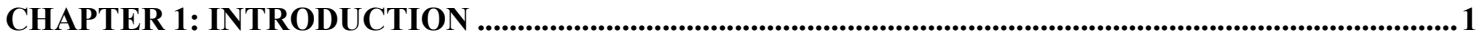

1.1 High-Throughrut Microscopy Confocal NeURon IMAGES ..............................................................

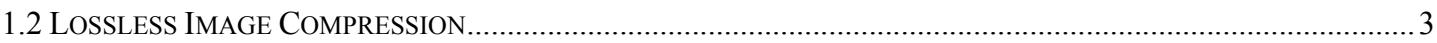

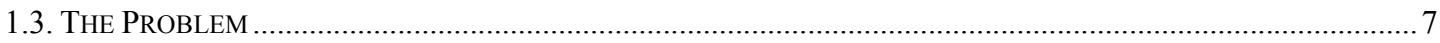

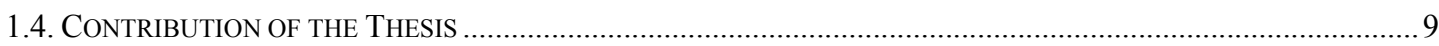

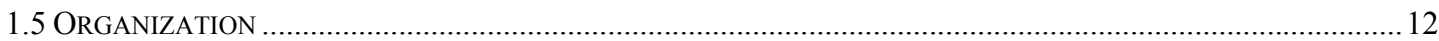

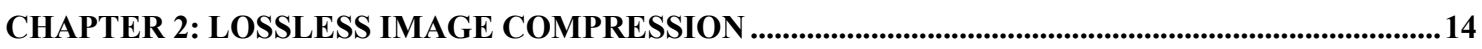

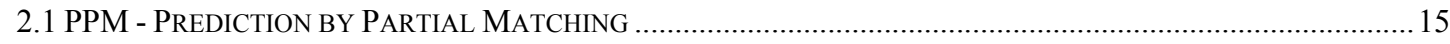

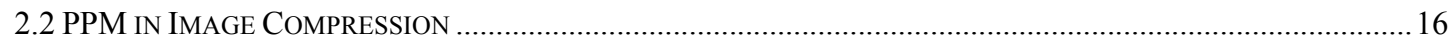

2.3 PPAM: Prediction By PARTIAL APPRoXimate MATCHING ........................................................... 17

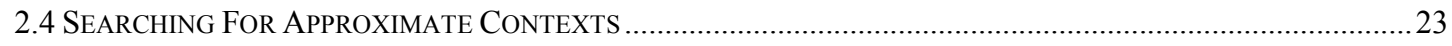

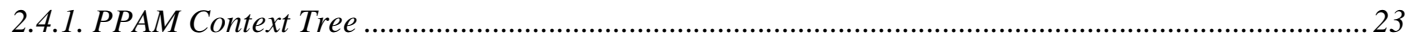

2.4.2. Searching for k-approximate contexts .....................................................................................24

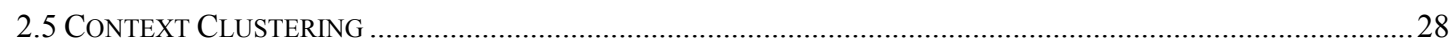

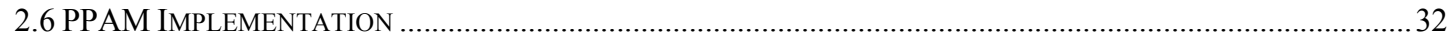

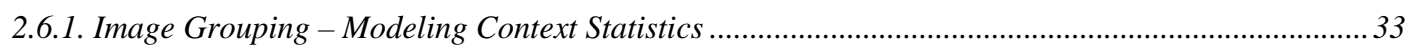

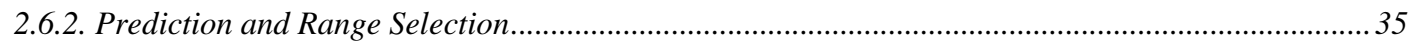

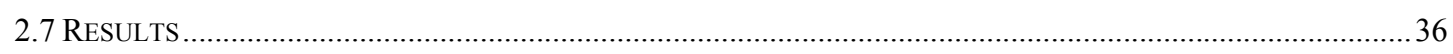

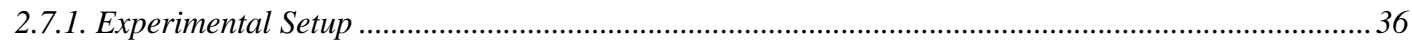

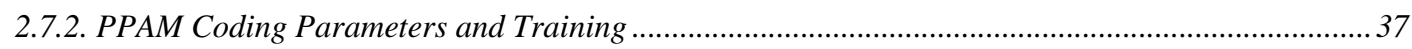

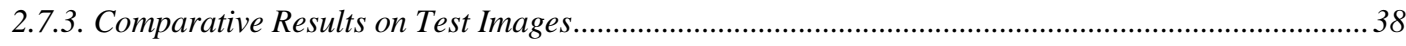

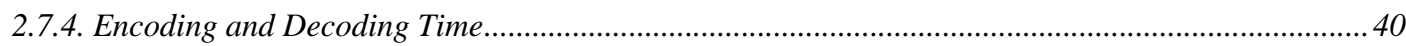

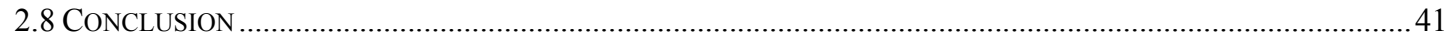




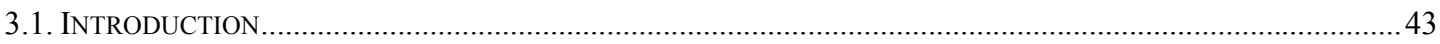

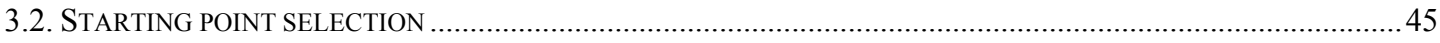

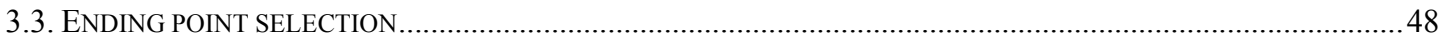

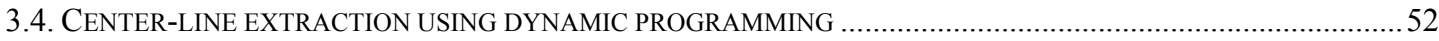

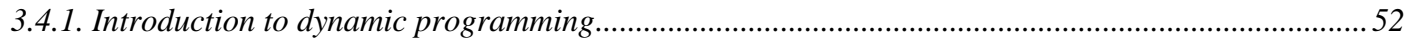

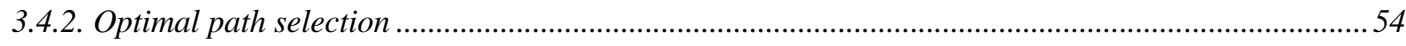

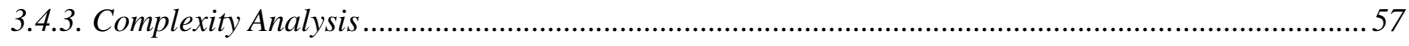

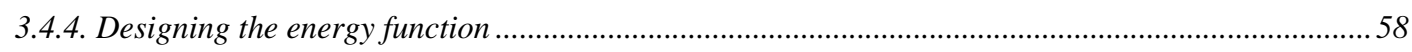

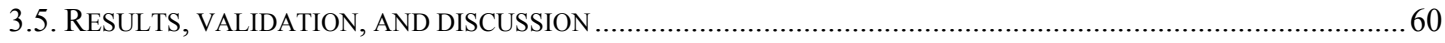

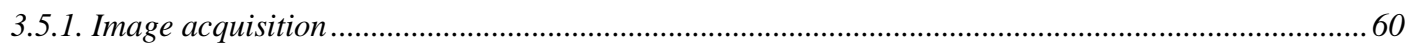

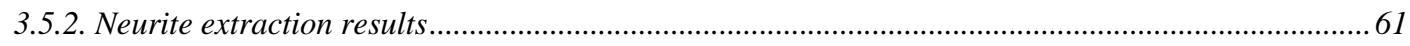

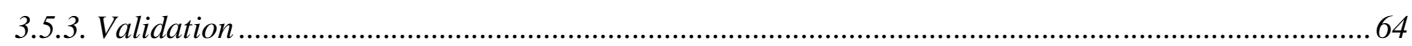

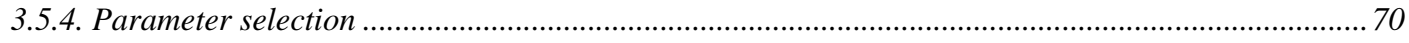

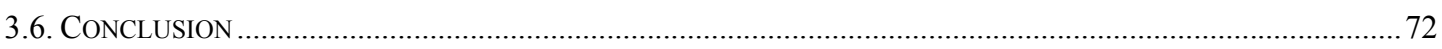

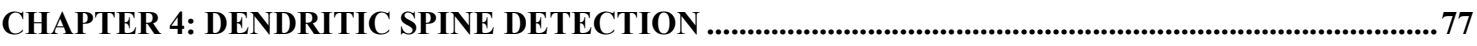

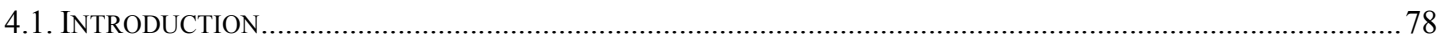

4.2. AutOMATED EXTRACTION OF DENDRITIC BACKBONES ........................................................................ 81

4.2.1. Centerline Extraction for Dendritic Backbones .........................................................................8

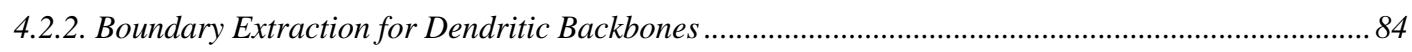

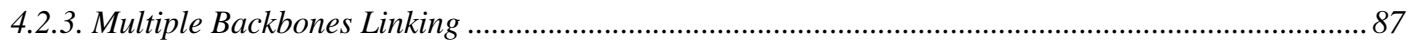

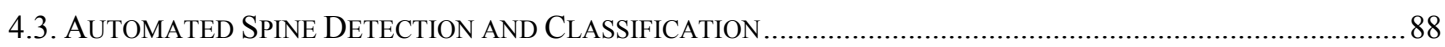

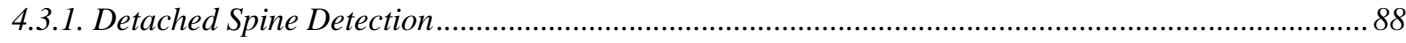

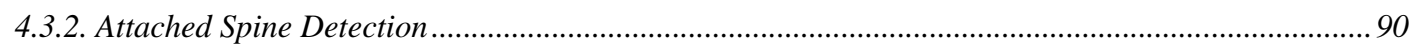

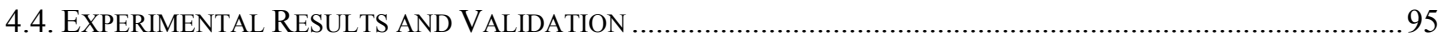

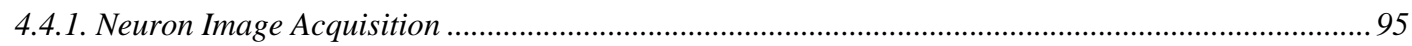

4.4.2. Results for Dendritic Backbone and Spine Extraction..............................................................95

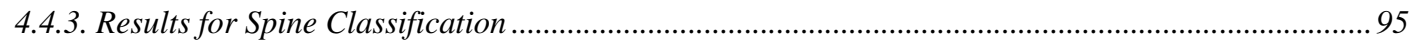

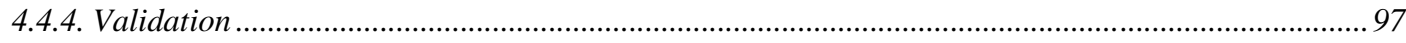

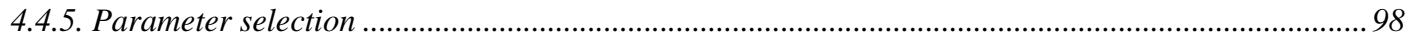


CHAPTER 5: 3D NEURITE CENTERLINE EXTRACTION ................................................................113

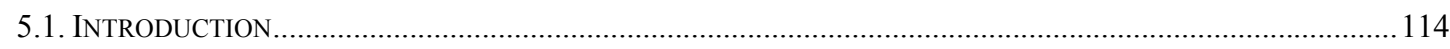

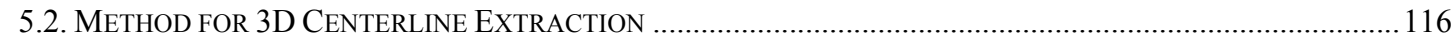

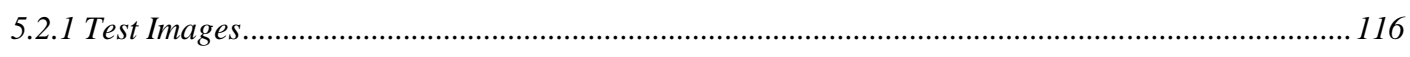

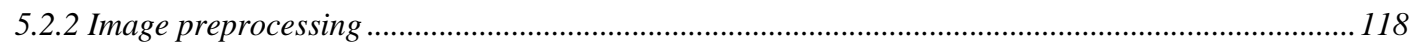

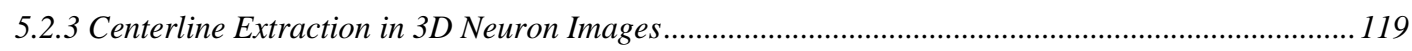

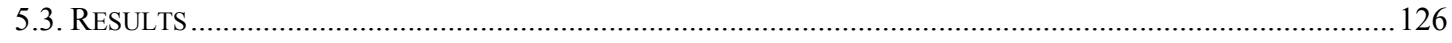

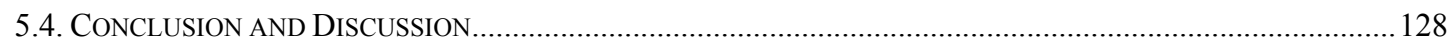

CHAPTER 6: CONCLUSION AND DISCUSSION ..............................................................................131

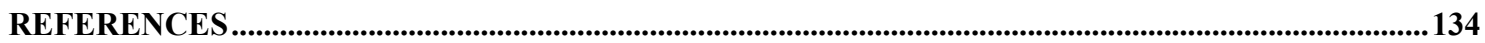




\section{List of Tables}

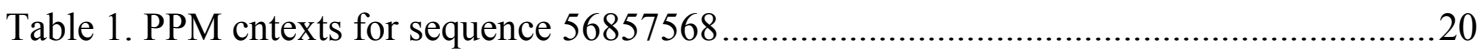

Table 2. SUMMARY OF PPAM CONTEXT RESULTS - CONTEXT COUNTS ..................................2

Table 3. PPAM contexts for sequence 56857568, [.] indicates set of $k$-approximate contexts

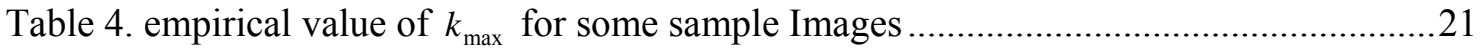

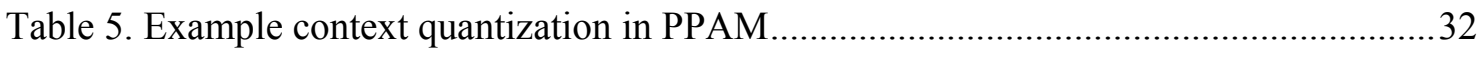

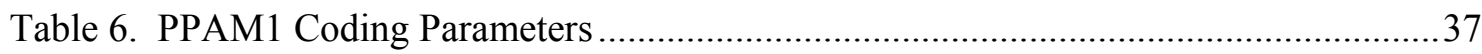

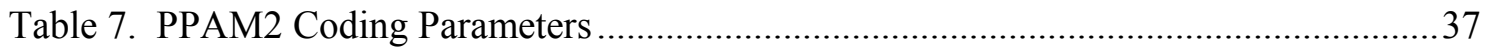

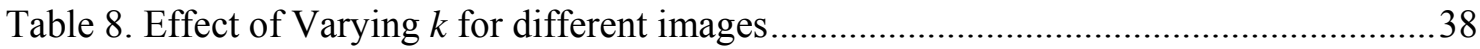

Table 9. Comparative Results with EDP and JPEG-LS (bits per pixel) ................................39

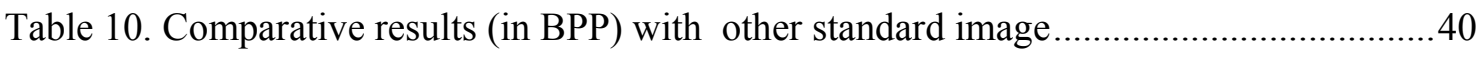

Table 11. Comparative results (in BPP) with other standard Compression Algorithms on neurite images. the representative image for PPAM was neurite02, denoted with “*”...40

Table 12. Encoding and decoding time for ppam 1 and ppam2 (in seconds) ........................41

Table 13. The mean and standard deviations of the length difference and centerline deviation, .66

Table 14. The Pearson linear correlation coefficients between the results generated from ....66

Table 15. SFFS feature selection performance for different number of features ...................96

Table 16. Comparison of spine number and average spine length measured by two methods 98

Table 17. Quantitative comparison of each individual test image ........................................99

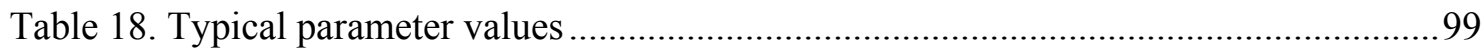




\section{List of Figures}

Figure 1. Sample neuron images (a) in $2 \mathrm{D}$, (b) in $3 \mathrm{D}$, projected on $\mathrm{y}$-plane............................

Figure 2. PPAM Context Tree. (a) Context tree for sample sequence $S=56857568 \ldots \ldots \ldots \ldots . .23$

Figure 3. Searching PPAM context tree using the grouping and marking algorithm.............27

Figure 4. Distribution of $k$-approximate contexts for LENNAGREY; (a) approximate context

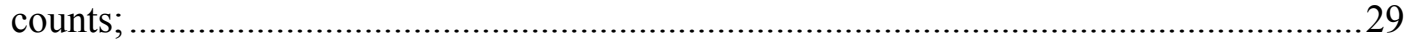

Figure 5. Contexts template for PPAM (a) order-4 context (b) order-5 context ....................30

Figure 6. PPAM Lossless Image Compression Scheme …....................................................33

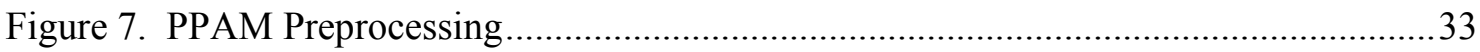

Figure 8 . The block diagram for the neurite tracing algorithm using DP ...........................45

Figure 9. The correlation kernel at zero-degree direction................................................49

Figure 10. A example of finding the optimal path using the DP ......................................55

Figure 11. Linking relationships between two points.......................................................56

Figure 12. Sample results for neurite extraction. (a) original image; (b) result image with neurites labeled by green color and soma regions labeled by red color.

Figure 13. Sample results for neurite extraction. (a) original image; (b) result image with neurites labeled by green color and soma regions labeled by red color.

Figure 14. A partial image of neurite segment and two extracting results. (a) original partial image; (b) extracting result using NeuronJ; (c) extracting result using our proposed method.

Figure 15. Box plots for the length difference and the centerline deviation...........................68

Figure 16. Quantile-quantile plots of the length of the neurite segments.............................69

Figure 17. Quantile-quantile plots of the length of the neurite segments............................. 70

Figure 18. Comparison between extractions using different standard deviation (STD) values

for the Gaussian kernel. 
Figure 19. Comparison between extractions using different weight values $(w)$ for the cost function.

Figure 20. Comparison between extractions using different weight values $(\varepsilon)$ for the cost

function. .76

Figure 21 A sample neuron image stack projected along 80

Figure 22 The block diagram for the automated backbone and spine detection .81

Figure 23 The 2D line model represented by a 1D line profile along the direction .82

Figure 24 Example results for boundary detection. .87

Figure 25 The block diagram for the spine classification using LDA .94

Figure 26. Results for dendritic backbone and spine extraction. 101

Figure 27. Results for dendritic backbone and spine extraction. 102

Figure 28. Partial spine samples in the training set for spine classifier. 103

Figure 29. Spine detection results. 104

Figure 30. Spine detection results. 105

Figure 31. (a)(b) Two Comparison results between our algorithm and the medial axis and global threshold based algorithm.

Figure 32. (a)(b) Two Comparison results between our algorithm and the medial axis and global threshold based algorithm. 109

Figure 33. Visual comparison. 111

Figure 34. Visual comparison: Two example images on which both automated and manual method obtain the same result. 112

Figure 35. The projection view (Maximum Intensity Projection) of a sample 3D neuron image...

Figure 36. An example on how the 3D neuron image stack is formed. 117 
Figure 37. Marker points selection. The two points in colors are the selected marker points

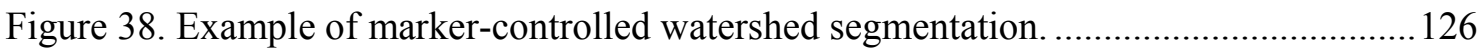

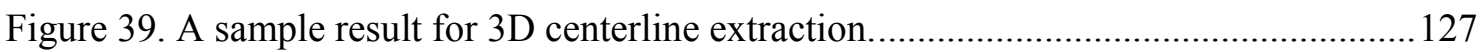

Figure 40. Partial 3D centerline extraction result to show the power of marker-controlled

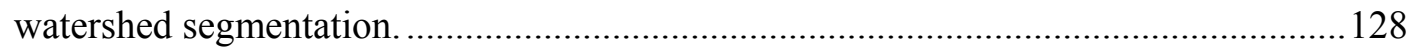

Figure 41. Partial 3D centerline extraction result to show the power of marker-controlled

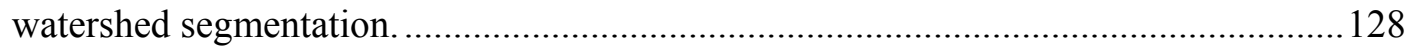

Figure 42. Two views of a 3D neuron image with very complicated neuron structures.......130 


\section{Chapter 1: Introduction}

\subsection{High-Throughput Microscopy Confocal Neuron Images}

Many researchers have devoted their time and efforts to identifying potential compounds that could prevent damages and losses of synapses and neurites, known to be the early events in Alzheimer's disease (AD). One predominant view of the primary cause of $\mathrm{AD}$ is the abnormal accumulation of the A-beta peptides, the primary component of the amyloid plaques. In order to study the role of A-beta, researchers have established experiments using sensitive and reproducible assays for amyloid neurotoxicity in mouse primary cortical neurons using either aggregated or soluble A-beta 1-40. Light microscopy was used to observe neuronal morphology following A-beta treatment as compared to untreated controls. Recently researchers at Dr.Yuan's Lab at Harvard Medical School have further extended the analyses of the neurite loss following A-beta treatment to the use of high-throughput screening methodology developed at the Institute of Chemistry and Cell Biology (ICCB). They utilized cortical neurons stained with neuronal-specific antibody MAP2 and normalized to neuronal cell number by counterstaining with nuclear stain DAPI. Primary cortical neurons were left untreated or treated with $10 \mu \mathrm{M}$ A-beta $1-40$ in 384 well plates for 48 hours, followed by staining with TUJ1 and Sytox Green. Cell images were acquired using Autoscope platereader at $20 \mathrm{X}$ resolution. A typical experiment may generate more than 23,040,000 images (10000 compounds $\times 384$ wells/plate $\times 6$ image fields/well).

As discussed above, current optical microscopy techniques, coupled with a large arsenal of fluorescent and other labeling methods, generate a tremendous number of images that needs to be quantitated. However, the tools for analyzing the images have not kept pace with these developments. While existing image analysis tools, such as NIH Image (available as 
Scion Image or Image/J), MetaMorph, UTHSCSA ImageTool, QED Image and CellProfiler, perform satisfactorily in processing neuron images, they are extremely limited in their scope and capability for high-throughput neuron image analysis, particularly with respect to complex neuron structures or multi-spectral correlations. The above tools cannot extract the full range of biological information from the highly complex images obtained by modern fluorescence microscopy. Fig. 1.1 shows two sample neuron images ${ }^{1}$.

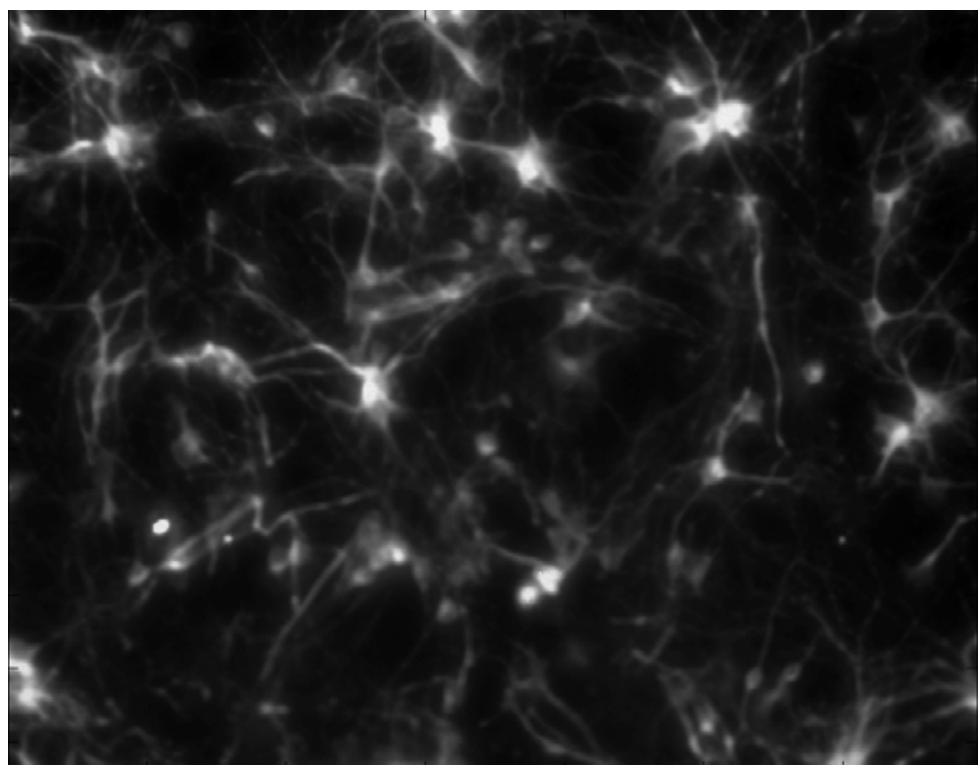

(a)

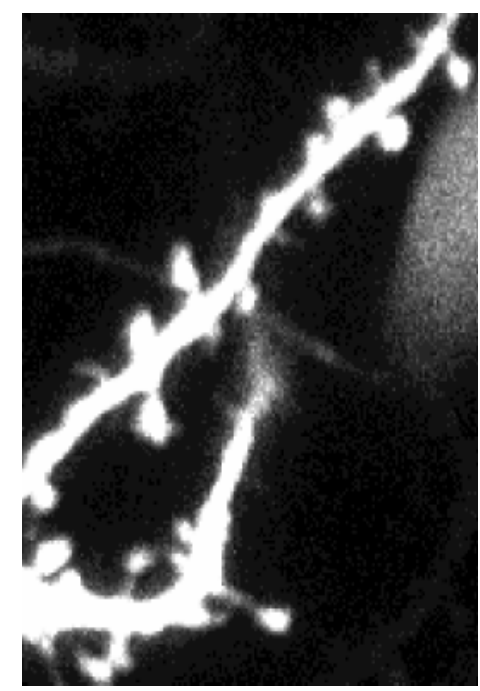

(b)

Figure 1. Sample neuron images (a) in 2D, (b) in 3D, projected on y-plane

There are three basic methods for extracting neuron structures. The direct exploratory tracing algorithms [1] automatically detect the initial points and extract the centerlines of the neuron structures in a tracing-based iterative manner. However, the tracing performance is

\footnotetext{
${ }^{1}$ The sample Neuron images are provided by Yuan's Lab, one of our neuroscience collaborators at the Institute of Chemistry and Cell Biology (ICCB), Harvard Medical School. ICCB has an excellent high-throughput small molecule screening facility, one of the first high-throughput screening facilities in academic centers.
} 
quite low for images with poor quality and for line structures with discontinuities, or when the image has complicated branching structures.

The second approach [2] explores the local geometric properties of the lines and examines for each pixel in the image, followed by linking the successive line pixels that are most likely to represent the centerlines of the neuron structures. The drawbacks of this algorithm are that: (1) it can only extract lines with a certain range of widths; (2) it requires more computation than the direct exploratory algorithms; and (3) it can only detect lines in high contrast areas.

Recently, a semi-automatic approach [3] has been proposed for 2D curvilinear neurite segments tracing that employed local principal ridge direction to guide the live-wire algorithm to track centerlines. Although robust against noise and discontinuities in line structures, these algorithms are unable to detect neurite segments with low contrast and segments with branching and crossover points. The requirement that the starting points and the end points be selected manually by the users is a major drawback of this approach especially for high throughput applications.

\subsection{Lossless Image Compression}

Image compression methods are usually classified as either lossy or lossless. Lossy compression schemes usually provide huge compression, but they often introduce irreversible errors in the reconstructed images. For some applications, (and especially for legal or medical reasons), images have to be stored or transported in such a way that they can be later recovered without any changes in the image. In lossless image compression, the image is compactly represented such that it can be reconstructed without any error.

Lossless image compression schemes can either treat the image as a one-dimensional text sequence, or they can make use of the two-dimensional contexts to improve the coding 
performance. The former approach involves an initial stage of two-dimensional to onedimensional conversion, and a text compression algorithm for compressing the onedimensional sequence. The most popular lossless image compression methods (such as GIF, TIFF and PNG) are based on Ziv-Lempel coding algorithm [4] after a raster scan of the image. This simple approach is remarkably effective for simple images.

Most successful lossless image compression algorithms are context-based and they exploit the two-dimensional spatial redundancy in natural images [5-10]. Available algorithms include FELICS [8], CALIC [5], JPEG-LS [11], JPEG2000, Glicbawls [12], TMW [13], and BMF. These methods usually involve four basic components: an initial prediction scheme to remove the spatial redundancy between neighboring pixels; a context selection strategy for a given position in the image; a modeling method for the estimation of the conditional probability distribution of the prediction error given the context in which it occurs; and an entropy coding method based on the estimated conditional probabilities. The different lossless image compression schemes vary in the details of one or more of the basic components.

The basic motivation for context-based approaches is the promise of improved compression. If contexts are considered, the conditional probability distribution for the set of symbols is used for the entropy coding procedure, which indeed reduces the number of bits needed to encode the image. Consider an image of size $N_{1} \times N_{2}$. Let the image be represented by a sequence $S=\left\{s_{i}, i=1, \ldots,|S|\right\}$, with symbols taken from a fixed alphabet $\Sigma=\left\{\sigma_{i}, i=1, \ldots,|\Sigma|\right\}$, where $|S|=N=N_{1} N_{2}$ is the image size. The symbol alphabet $\Sigma$ is typically the set of distinct pixel gray levels in the image, or the set of distinct prediction errors, after applying some prediction scheme. Let the corresponding probability distribution 
of the symbols be $p\left(\sigma_{i}\right), i=1, \ldots,|\Sigma|$. Then the minimum number of bits per symbol required to encode the image without context modeling is the entropy of the source $H(S)$ :

$$
H(S)=-\sum_{\sigma_{i} \in \Sigma} p\left(\sigma_{i}\right) \log _{2} p\left(\sigma_{i}\right)
$$

If contexts are considered, the conditional probability distribution for the set of symbols $S_{j}^{\prime}$

with the context $C_{j}$ will be $p\left(s_{i} \mid C_{j}\right), i=1, \ldots\left|S_{j}^{\prime}\right|$, and the minimum number of bits per symbol needed to encode the source should be:

$$
\begin{aligned}
& H(S \mid C)=-\sum_{j=1}^{M}\left(p\left(C_{j}\right) \sum_{s_{i} \in S_{j}^{\prime}} p\left(s_{i} \mid C_{j}\right) \log _{2}\left(p\left(s_{i} \mid C_{j}\right)\right)\right) \\
& H(S \mid C)=-\sum_{j} \sum_{s_{i}} p\left(s_{i}, C_{j}\right) \log _{2}\left(p\left(s_{i} \mid C_{j}\right)\right)
\end{aligned}
$$

where $M$ is the total number of contexts. Since conditioning reduces entropy, the number of bits per symbol needed to encode the image is also reduced by using context modeling:

$$
H(S \mid C) \leq H(S)
$$

Although the lossless image coding algorithms are based on some form of contexts, they always tend to use fixed-order contexts, which may limit their ability in estimating the conditional probability distributions. On the other hand, the most successful text compression schemes use variable-order contexts in compressing the text. It is therefore natural to investigate how similar ideas can be used for lossless image compression, especially exploiting the potentially large number of similar contexts and other special characteristics of natural images. In particular, we consider how images can be coded using ideas from the Prediction by Partial Matching (PPM) family of text compression algorithms [14-16]. In text compression, the PPM family of compression algorithms is known to produce the best results in terms of data compaction ability. Various variants of the PPM model exist, such as PPMA, PPMB, PPMC, PPMD, PPM*, etc [14-16]. The models differ mainly in the way the escape 
probability is determined, and in how they handle the zero-frequency problem [17]. The data in Table I(a) are based on the PPMC model [15], where probabilities are assigned as follows:

$$
P(\operatorname{esc} \mid C)=\frac{a}{a+c}
$$

where $a$ is the number of distinct symbols so far observed in the context $C$, and $c$ represents the total number symbols in the context $C$. Under the PPMC model, symbol $s$ with $b$ counts in context $C$ will be assigned the probability

$$
P(s \mid C)=\frac{b}{a+c}
$$

There has also been some effort to use PPM in image compression. In [18], the PPMD model was used to compress the prediction error sequence from multi-spectral satellite images. No special attention was paid to the PPM context model, which was used as a simple black box. Context-tree based methods for compressing map images have also been proposed [19]. PMIC - a pattern matching based scheme for lossy image compression was proposed in [20]. The context tree method and PMIC are related work, but not necessarily based on the PPM.

Much earlier, Howard and Vitter proposed PPPM - prediction by partial precision matching for image compression [9]. Here, searching for similar contexts was based on similarity rather than on exact matches. In searching for similar contexts, if the current context of a pixel has not occurred enough times in the past, PPPM switches to a less precise context. The contexts are represented as binary sequences, and stored using a hash table. To use a less precise context, the least significant bit of each symbol in the current context is dropped, and the reduced-size binary sequence is used to form a new context. This essentially quantizes the old context by dividing the value by 2 . Context searching is then performed using this new context. This iterative division process continues until a matching similar context is found. The encoder then computes the mean and variance based on the similar 
contexts. These are then used to select from 37 pre-stored Laplace distribution tables to determine an estimate of the conditional probability distribution at the current coding step. The tables are similar to the coding tables used in FELICS [8]. A major problem with the PPPM scheme is the amount of error that could be introduced, especially with increasing levels of iteration. Thus, the real problem of extending PPM to context modeling for images still remains unresolved.

Recent research progress in biomedical image compression has shown increasing interest in compressing medical volumetric data using integer wavelet transform based methods in a lossy-to-lossless manner [21, 22]. Bernas et al. [23] has addressed the problem of compressing fluorescence microscopy images in the lossy manner. However, not very much work has been done on the lossless compression of high-throughput microscopy confocal images, due to the fact that the high-throughput is a new imaging technique adopted only recently in the field of biomedical research.

\subsection{The Problem}

There exist two key problems on neuron images. The first problem is on the compression and storage of the huge volume of the image data. Existing lossless compression schemes may not be suitable for such type of image since they are designed to deal with regular or natural images. However for neuron images, the images acquired from one experiment can have similar neuron structures and they are correlated to each other. Thus, it is possible to explore such properties of the neuron images and design a novel context based modeling method to improve the compression performance.

Context modeling is a difficult problem in image compression. At any given position in an image, there are a potentially huge number of possible contexts. Thus, a given context may not have occurred previously and the probability of finding this single context out of the huge 
number of possible contexts will be very small. The result is that, under the PPM model, most of the symbols will be coded using a sequence of escape symbols, which will lead to a significant loss in compression. This means that the traditional PPM model will not be an effective model for contexts in natural images. Further, edges are one of the important properties of natural images. Unless there is a strong edge boundary, natural images usually contain locally homogeneous areas, with such local neighborhoods having a strong recency effect. However, the repetition within these local neighborhoods is usually not exact. The repetitions often occur in an approximate form. This implies that, for images, though there could be a large number of similar contexts, the contexts may not repeat in an exact form. For instance, unlike in text, two contexts $C_{1}=[100,67,89,205]$ and $C_{2}=[98,69,91,204]$ can be regarded as similar contexts in an image, although they are not exact matches.

Another problem with context modeling in images is that of noise. When images are contaminated by random noise signals, it becomes more difficult to find contexts that are repeated in an exact manner in the image, since the original pixel values could be slightly changed by the noise signal in an unpredictable manner. Thus it may be more appropriate to model the contexts in such noisy images by considering the context occurrences in an approximate form, rather than as exact occurrences.

We present a different approach to the problem of context matching with errors, while still recovering the coded symbol in a lossless manner. We call this method PPAM prediction by partial approximate matching. Our method is motivated by the characteristics of natural images, and by ideas from approximate pattern matching in text [24, 25]. We show that, in a sense, the proposed approach can be viewed as a generalization of the PPPM context model and a modification and extension of the PPM algorithm to lossless image compression. 
The second problem is on neuron image analysis. Despite the considerable effort to automate this task during the past decade, the attempts are not successful due to lack of public available image databases and inevitable human interaction. The analysis usually includes detection of neuron structures, feature extraction, quantification, and statistical analysis. For this purpose, we develop novel methods for extracting curvilinear neurite segments and dendritic spines in 2D/3D neuron images and for extracting axons in 3D neuron images. We also study effective validation methods for evaluating the performance of the proposed neuron structure extraction algorithms. The developed algorithms are able to automatically, rapidly, accurately, completely, and robustly extract neuron structures, and require only minimum human interaction.

\subsection{Contribution of the Thesis}

For the problem of lossless neuron image compression, we start from studying the most successful context modeling method for text data compression. The method is called Prediction by Partial Matching (PPM). We then see how similar ideas can be used for lossless compression of natural images, especially exploiting the potentially large number of similar contexts and other special characteristics of natural images. When considering how images can be coded using ideas from the PPM family of text compression algorithms, we explore two key questions: how to effectively handle the huge number of contexts in images and how to condition the encoding symbols (prediction errors) given the context. We present PPAM Prediction by Partial Approximate Matching, a method for compression and context modeling for images. Unlike the PPM modeling method that uses exact contexts, PPAM introduces the notion of approximate contexts. Thus, PPAM models the probability of the encoding symbol based on its previous contexts, whereby context occurrences are considered in an approximate manner. The idea of approximate contexts is more suitable for context 
modeling in images. The proposed method has competitive compression performance when compared with other popular lossless image compression algorithms. We extend the modeling method to compressing neuron images and obtain particularly superior performance when compared with other state-of-the-art compression schemes. The PPAM can be easily extended to compress other types of images that share common features, for example, medical images, aerial images, and so on.

For the problem of extracting curvilinear neurite segments in 2D neuron images, we start by studying existing methods and evaluate their advantages and disadvantages. The test neuron images are first pre-processed to remove noise, discard undesirable features, and correct illumination artifacts. Thus the desired image features can be enhanced for further analysis. We study both the direct exploratory tracing based method and line-point detection based method and try to combine their advantages. We present a novel algorithm for extraction and quantification of neurite segments from HTS neuron images. The algorithm is designed to be able to detect and link neurites even with complex neuronal structures and of poor imaging quality. Our algorithm automatically detects initial seed points on a set of grid lines and estimates the ending points of the neurite by iteratively tracing the centerline points along the line path representing the neurite segment. The live-wire method is then applied to link the seed points and the corresponding ending points using dynamic programming techniques, thus enabling the extraction of the centerlines of the neurite segments accurately and robustly against noise, discontinuity, and other image artifacts. A fast implementation of our algorithm using dynamic programming is also discussed in the thesis. Any thin neurite and its segments with low intensity contrast can be well preserved by detecting the starting and ending points of the neurite. All these properties make the proposed algorithm attractive for high-throughput screening of neuron-based assays. We develop a validation methodology to quantitatively evaluate the extraction performance of the proposed algorithm. 
We propose a novel approach for automated detection of dendritic spines in neuron images. The dendritic spines are treated as small protrusions of variable shape attached or detached to multiple dendritic backbones in the $2 \mathrm{D}$ projection of the image stack along the optical direction. We extend the curvilinear structure detector to extract the boundaries as well as the centerlines for the dendritic backbones and spines. We further build a classifier using Linear Discriminate Analysis (LDA) to classify the attached spines into valid and invalid types to improve the accuracy of the spine detection. We evaluate the proposed approach by comparing with the manual results in terms of backbone length, spine number, spine length, and spine density.

For the problem of extracting 3D neurite structures, we develop a semi-automatic method for extracting $3 \mathrm{D}$ neurite structures. With the semi-automatic method, the user needs to assign the starting point for each major neurite segment, and the centerlines of the neurite segments are automatically detected by the algorithm. This significantly reduces the complexity of the algorithm and improves accuracy in further analysis, due to the observation that the number of neurite segments in the $3 \mathrm{D}$ neuron images is usually very small. We propose a highly automated $3 \mathrm{D}$ centerline extraction tool to assist in this task. We track the 3D curvilinear structure detection using the 3D dynamic programming techniques. Starting from a set of pre-defined seed points, we search for optimal paths for the points that are within a small region in the $3 \mathrm{D}$ space. The points on the optimal paths with the minimum cost values are regarded as the foreground markers and the marker-controlled watershed algorithm is applied to segment the slice into different axon objects. The centroids of the segmented regions are used as the extracted centerline points. The proposed method can handle complicated axon structures such as cross-over sections and attaching segments. 


\subsection{Organization}

We focus on the problems related to microscopy confocal neuron images. For neuron image compression, we introduce novel context modeling method based on Prediction by Partial Approximate Matching (PPAM). Unlike the Prediction by Partial Matching (PPM) modeling method which considers the contexts precisely, PPAM models the probability of the encoding symbol from its previous contexts with which the context occurrences are considered in an approximate manner. For neuron structure extraction, we aim at robust and efficient feature extraction algorithm. Robustness comes from the use of curvilinear structure detection and estimation of surrounding edge points. Efficiency is achieved by processing only pixels that are close enough to the line structures, and by carefully chosen stopping conditions. These make the proposed approach suitable for demanding image processing tasks in high throughput screening of neuron-based assays.

Chapter 2 introduces PPAM - Prediction by Partial Approximate Matching, a method for compression and context modeling for neuron images, motivated by the PPM context model used in text compression. We also introduce an efficient context searching method using a PPAM context tree. Further analysis shows that exhaustive searching can be improved by performing searches on multiple context trees. We apply the proposed compression method on neuron images. We apply the PPAM method to compress natural images as well as neuron images.

Chapter 3 introduces a new algorithm for fast and automatic extraction of neurite structures in 2D microscopy neuron images. The algorithm is based on novel methods for soma segmentation, seed point detection, recursive center line detection, 2D curve smoothing, and centerline point linking using dynamic programming techniques.

Chapter 4 discusses a novel method for automated detection of dendritic backbones and spines suitable for 3D neuron images in thin slices. Our method treats the dendritic 
backbones and spines as curvilinear structures and extracts their centerlines and boundaries by estimating the second order directional derivatives. A classifier is built from a pre-selected training set and the detected spines are further classified to remove pseudo spines.

Chapter 5 introduces the algorithm for detecting the centerlines of multiple branching axons in 3D neuron image stack. The 3D curvilinear structure extraction mimics the centerline tracing procedure in $3 \mathrm{D}$ space as walking along a path with minimized cost value. The cost function is defined following the three basic rules, namely, smoothness, proximity and continuity. Marker-controlled watershed algorithm is applied to segment the axons in each slice so that the touching axons can be separated from each other.

Chapter 6 presents the conclusions and discussions of the overall work. Potential future work is also discussed. 


\section{Chapter 2: Lossless Image Compression}

\section{Related papers by Yong Zhang for this Chapter:}

(1) Y. Zhang and D. Adjeroh, "Prediction by partial approximate matching for lossless image compression," in IEEE Computer Society Data Compression Conference Snowbird, Utah, 2005.

(2) Y. Zhang, R. Parthe, and D. Adjeroh, "Lossless compression of DNA microarray images," in IEEE Computational Systems Bioinformatics Conference Stanford, CA, 2005.

(3) D. Adjeroh, Y. Zhang, and R. Parthe, "On denoising and compression of DNA microarray images," Pattern Recognition, vol. 39, pp. 2478-2493, 2006.

(4) Y. Zhang and D. Adjeroh, "Prediction by partial approximate matching for lossless image compression," IEEE Transactions on Image Processing, under review, 2006. 


\subsection{PPM - Prediction by Partial Matching}

In text compression, the PPM family of compression algorithms is known to produce the best results in terms of data compaction ability. The PPM compression scheme uses a symbol-wise model that adaptively generates the statistics of the text as the text is being compressed. Given a current symbol $s$, and its $m$-th order context $C=c_{m} c_{m-1} \ldots c_{2} c_{1}$, a key problem in symbol-wise compression schemes is to generate a prediction of $s$ based on its context $C$. These predictions are used to compute the conditional probability $P(s \mid C)$ for each input symbol, which is then used by an entropy encoder.

Rather than using a single fixed-order context, the PPM scheme uses a set of finite order contexts. By using a blending scheme, it carefully switches from the highest to the lower order contexts, depending on the input data. Sequential blending of the contexts is performed by using escape symbols. Let $m_{\max }$ be the maximum order context used. At each coding step, PPM tries to use its maximum order context to predict the next symbol. If the symbol has appeared previously in this context, the symbol is coded with $P\left(s \mid c_{m_{\max }} c_{m_{\max }-1 \ldots c_{1}}\right)$. If the symbol has never appeared in this context, an escape symbol is sent, and the context order is reduced to $m_{\max }-1$. The process is repeated with this reduced-order context. If the model reaches the zeroth-order context, the symbol is encoded based on a fixed pre-determined probability distribution. This procedure is shown in Table I(a) (modified from [15]), for the sequence abracabr. Here, for ease of presentation and comparison, we have modified the sequence using the following mapping to integers: $a \rightarrow 5 ; b \rightarrow 6 ; c \rightarrow 7 ; r \rightarrow 8$. This gives the sequence: $S=56857568$.

Various variants of the PPM model exist, such as PPMA, PPMB, PPMC, PPMD, PPM*, etc [14-16]. The models differ mainly in the way the escape probability is determined, and in how they handle the zero-frequency problem [17]. The data in Table I(a) are based on the 
PPMC model [15], where probabilities are assigned as follows:

$$
P(e s c \mid C)=\frac{a}{a+c}
$$

where $a$ is the number of distinct symbols so far observed in the context $C$, and $c$ represents the total number symbols in the context $C$. Under the PPMC model, symbol $s$ with $b$ counts in context $C$ will be assigned the probability

$$
P(s \mid C)=\frac{b}{a+c}
$$

\subsection{PPM in Image Compression}

There has also been some effort to use PPM in image compression. In [18], the PPMD model was used to compress the prediction error sequence from multi-spectral satellite images. No special attention was paid to the PPM context model, which was used as a simple

black box. Context-tree based methods for compressing map images have also been proposed [19]. PMIC - a pattern matching based scheme for lossy image compression was proposed in [20]. The context tree method and PMIC are related work, but not necessarily based on the PPM.

Much earlier, Howard and Vitter proposed PPPM - prediction by partial precision matching for image compression [9]. Here, searching for similar contexts was based on similarity rather than on exact matches. In searching for similar contexts, if the current context of a pixel has not occurred enough times in the past, PPPM switches to a less precise context. The contexts are represented as binary sequences, and stored using a hash table. To use a less precise context, the least significant bit of each symbol in the current context is dropped, and the reduced-size binary sequence is used to form a new context. This essentially quantizes the old context by dividing the value by 2 . Context searching is then performed using this new context. This iterative division process continues until a matching similar 
context is found. The encoder then computes the mean and variance based on the similar contexts. These are then used to select from 37 pre-stored Laplace distribution tables to determine an estimate of the conditional probability distribution at the current coding step. The tables are similar to the coding tables used in FELICS [8]. A major problem with the PPPM scheme is the amount of error that could be introduced, especially with increasing levels of iteration.

We present a different approach to the problem of context matching with errors, while still recovering the coded symbol in a lossless manner. We call this method PPAM prediction by partial approximate matching. Our method is motivated by the characteristics of natural images, and by ideas from approximate pattern matching in text [24, 25]. We show that, in a sense, the proposed approach can be viewed as a generalization of the PPPM context model and a modification and extension of the PPM algorithm to lossless image compression.

\subsection{PPAM: Prediction by Partial Approximate Matching}

At any given position in an image, we have a potentially huge number of possible contexts. Thus, a given context may not have occurred previously and the probability of finding this single context out of the huge number of possible contexts will be very small. The result is that, under the PPM model, most of the symbols will be coded using a sequence of escape symbols, which will lead to a significant loss in compression. This means that the traditional PPM model will not be an effective model for contexts in natural images. Further, edges are one of the important properties of natural images. Unless there is a strong edge boundary, natural images usually contain locally homogeneous areas, with such local neighborhoods having a strong recency effect. However, the repetition within these local neighborhoods is usually not exact. The repetitions often occur in an approximate form. For 
instance, unlike in text, two contexts $C_{1}=[100,67,89,205]$ and $C_{2}=[98,69,91,204]$ can be regarded as similar contexts in an image, although they are not exact matches. Further, when images are contaminated by random noise signals, it becomes even more difficult to find repeated exact contexts. For such noisy images, modeling contexts in an approximate sense may be more appropriate.

In context-based compression schemes, the key to compression is to condition the probability of a symbol based on its current context. This conditional probability will typically be greater than the order-zero probability of the symbol, and hence would lead to a better compression, (see Section I). Thus, a compression algorithm that can recognize the potentially approximate nature of the contexts in an image is more likely to produce a more appropriate conditional probability distribution for a given context, and hence should perform better than those that depend on exact contexts.

In PPAM, we exploit these inexact contexts using methods from text pattern matching with errors. Given two symbols $s_{1}$ and $s_{2}$, we say that $s_{1}$ is a k-approximate match to $s_{2}$ if $\left|s_{1}-s_{2}\right| \leq k$, where $k$ is an integer value specified by the algorithm. We generalize this to context strings. Given two order-m contexts $C_{q}=c_{q}^{m} c_{q}^{m-1} \ldots c_{q}^{1}$ and $C_{d}=c_{d}^{m} c_{d}^{m-1} \ldots c_{d}^{1}$, we define the potential $k$-approximate contexts for $C_{q}$ and $C_{d}$ respectively as follows:

$$
\begin{aligned}
& S_{q}=\left\{\left(c_{q}^{m} \pm k\right) \circ\left(c_{q}^{m-1} \pm k\right) \circ \cdots \circ\left(c_{q}^{1} \pm k\right)\right\} \\
& S_{d}=\left\{\left(c_{d}^{m} \pm k\right) \circ\left(c_{d}^{m-1} \pm k\right) \circ \cdots \circ\left(c_{d}^{1} \pm k\right)\right\}
\end{aligned}
$$

where "。" represents the concatenation operator between two sets, and $c_{i} \pm k$ represents the set of symbols $c_{j}$ that are within a $k$-distance from $c_{i}$, that is, $c_{i} \pm k=\left\{c_{j},\left|c_{i}-c_{j}\right| \leq k\right\}$. For two sets $A$ and $B$, the concatenation operation is defined as follows: $A \circ B=\left\{a_{i} \circ b_{j}, \forall a_{i} \in A, b_{j} \in B\right\}$. In the PPM model, context-searching starts by looking for the maximum order context, and then escapes to shorter contexts until a match is found. In PPAM, we adopt a different 
approach, and search for $k$-approximate contexts to the current context with different values of $k$. Using the notations above, the problem is to find all the contexts $C_{j}$, such that $C_{j} \in S_{d} \wedge C_{j} \in S_{q}$. This is essentially a search problem, where we wish to find the size of the intersection defined by $S_{d} \cap S_{q}$. Formally, given two order- $m$ contexts, $C_{q}=c_{q}^{m} c_{q}^{m-1} \ldots c_{q}^{1}$ and $C_{d}=c_{d}^{m} c_{d}^{m-1} \ldots c_{d}^{1}$, we say that $C_{q}$ is a $k$-approximate match to $C_{d}$ iff

$$
\left|c_{d}^{m}-c_{q}^{m}\right| \leq k \wedge\left|c_{d}^{m-1}-c_{q}^{m-1}\right| \leq k \wedge \cdots \wedge\left|c_{d}^{1}-c_{q}^{1}\right| \leq k
$$

We note that this definition differs from that used in standard $k$-approximate matching, as used in text pattern matching $[24,25]$. While standard approximate pattern matching considers mainly the symbol positions (example "aabc" and "aadc" are 1-approximate matches), here, the symbols have values (example the grey-scale values or prediction errors), which have to be equally considered. Under PPAM, we start with exact matches $(k=0)$, and search for 0-approximate contexts (i.e. exact contexts). If the number of 0 -approximate contexts is smaller than a pre-determined threshold, $k$ is incremented by $1(k=1)$, and the search restarts with the new $k$ value. This process continues until we find enough number of $k$-approximate contexts, or $k$ reaches a maximum allowable error, $k_{\max }$. (Typically, $k_{\max } \leq 8$ ). The statistics obtained are then used to estimate the conditional probability distributions for the symbols. Thus, in PPAM, when the $k$-approximate context is not found, rather than escaping to a lower order context as in PPM, we transition to the next higher value of $k$. Hence, with the PPAM model, we do not need to send escape symbols, since the decoder can repeat the same search steps as the encoder.

Table 3 shows the PPAM contexts for the sequence $S=56857568$ used for PPM in Table 1. Table 2 presents the PPAM context counts for the same sequence. The search is based on contexts with fixed order $(m=2)$ and variable $k\left(k=0,1, \ldots, k_{\max }, k_{\max }=2\right)$. The notation $[C]$ represents the set of order- $m$ contexts that are $k$-approximate matches to context $C$ in the 
sequence. For example, using the sequence $S$, with $m=2$, and $k=1$ we will have: $[56]=\{56,57\}$.

Under $k$-approximate contexts, different $k$ values may lead to different compression results. Very low values of $k$ may limit our ability in observing the potential approximate contexts in natural images. However, large values of $k$ may involve too many approximate occurrences for all the contexts, and hence reduce the compression. Therefore, the error parameter $k$ has an important effect on the performance of PPAM. Table 4 shows the empirical value of $k_{\max }$ for some sample images. Observe the variation of $k_{\max }$ with the entropy or complexity of the image. In general, higher $k_{\max }$ values are expected for those images that are difficult to compress (with higher entropy). See also the section on compression results.

Table 1. PPM cntexts for sequence 56857568 $\mathrm{c}=$ number of occurrences; $\mathrm{p}=$ estimated probability

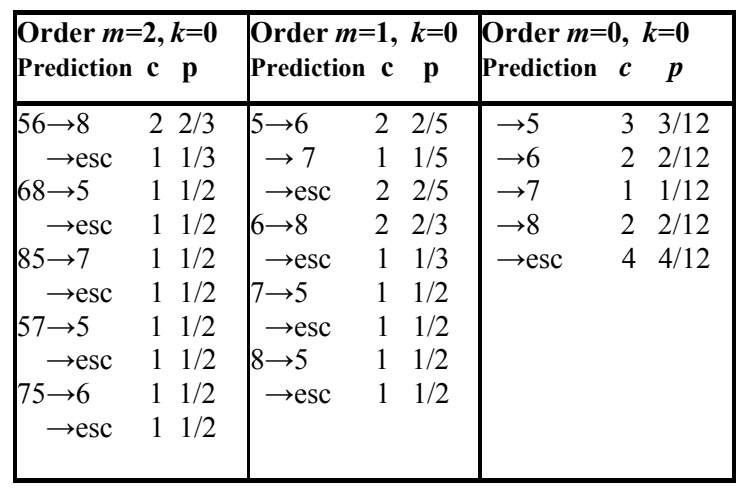

Table 2. SUMMARY OF PPAM CONTEXT RESULTS - CONTEXT COUNTS

\begin{tabular}{|l|l|l|l|}
\hline context & $\boldsymbol{k}=\mathbf{0}$ & $\boldsymbol{k}=\mathbf{1}$ & $\boldsymbol{k}=\mathbf{2}$ \\
\hline 56 & 2 & 3 & 6 \\
\hline 68 & 1 & 2 & 4 \\
\hline 85 & 1 & 2 & 2 \\
\hline 57 & 1 & 3 & 5 \\
\hline 75 & 1 & 2 & 6 \\
\hline
\end{tabular}


Table 3. PPAM contexts for sequence 56857568, [.] indicates set of $k$-approximate contexts to current context, $c=$ number of occurrences; $p=$ estimated probability

\begin{tabular}{|c|c|c|c|c|c|c|}
\hline \multirow{2}{*}{\multicolumn{2}{|c|}{$\begin{array}{l}\text { Order } m=2, k=0 \\
\text { Prediction } c p p\end{array}$}} & \multicolumn{3}{|c|}{ Order $m=2, k=1$} & \multicolumn{2}{|c|}{ Order $m=2, k=2$} \\
\hline & & Prediction & $c$ & & Prediction & $c \quad p$ \\
\hline $\begin{aligned} 56 & \rightarrow 8 \\
& \rightarrow \text { esc }\end{aligned}$ & $\begin{array}{ll}2 & 2 \\
1 & 1\end{array}$ & $\begin{array}{l}{[.] \rightarrow 5} \\
\rightarrow 8 \\
\rightarrow \mathrm{esc}\end{array}$ & $\begin{array}{ll}1 & 1 \\
2 & 2 \\
2 & 2\end{array}$ & $\begin{array}{l}1 / 5 \\
2 / 5 \\
2 / 5\end{array}$ & $\begin{array}{l}{[.] \rightarrow 5} \\
\rightarrow 6 \\
\rightarrow 8 \\
\rightarrow \text { esc }\end{array}$ & $\begin{array}{cc}2 & 2 / 8 \\
1 & 1 / 8 \\
2 & 2 / 8 \\
3 & 3 / 8\end{array}$ \\
\hline $\begin{aligned} 68 & \rightarrow 5 \\
& \rightarrow \text { esc }\end{aligned}$ & $\begin{array}{ll}1 & 1 \\
1 & 1\end{array}$ & $\begin{aligned} {[.] } & \rightarrow 5 \\
& \rightarrow \text { esc }\end{aligned}$ & $\begin{array}{ll}2 & 2 \\
1 & 1\end{array}$ & $\begin{array}{l}2 / 3 \\
1 / 3\end{array}$ & $\begin{aligned} {[.] } & \rightarrow 5 \\
& \rightarrow 8 \\
& \rightarrow \mathrm{esc}\end{aligned}$ & $\begin{array}{ll}2 & 2 / 6 \\
2 & 2 / 6 \\
2 & 2 / 6\end{array}$ \\
\hline $\begin{aligned} 85 & \rightarrow 7 \\
& \rightarrow \text { esc }\end{aligned}$ & $\begin{array}{ll}1 & 1 \\
1 & 1\end{array}$ & $\begin{aligned} {[.] } & \rightarrow 6 \\
& \rightarrow 7 \\
& \rightarrow \mathrm{esc}\end{aligned}$ & $\begin{array}{ll}1 & 1 \\
1 & 1 \\
2 & 2\end{array}$ & $\begin{array}{l}1 / 4 \\
1 / 4 \\
2 / 4\end{array}$ & $\begin{aligned} {[.] } & \rightarrow 6 \\
& \rightarrow 7 \\
& \rightarrow \mathrm{esc}\end{aligned}$ & $\begin{array}{ll}1 & 1 / 4 \\
1 & 1 / 4 \\
2 & 2 / 4\end{array}$ \\
\hline $\begin{aligned} 57 & \rightarrow 5 \\
& \rightarrow \text { esc }\end{aligned}$ & $\begin{array}{ll}1 & 1 \\
1 & 1\end{array}$ & $\begin{aligned} {[.] } & \rightarrow 5 \\
\rightarrow & \rightarrow 8 \\
& \rightarrow \mathrm{esc}\end{aligned}$ & $\begin{array}{ll}1 & 1 \\
2 & 2 \\
2 & 2\end{array}$ & $\begin{array}{l}1 / 5 \\
2 / 5 \\
2 / 5\end{array}$ & $\begin{array}{l}{[.] \rightarrow 5} \\
\rightarrow 6 \\
\rightarrow 8 \\
\rightarrow \text { esc }\end{array}$ & $\begin{array}{cc}2 & 2 / 8 \\
1 & 1 / 8 \\
2 & 2 / 8 \\
3 & 3 / 8\end{array}$ \\
\hline $\begin{aligned} 75 & \rightarrow 6 \\
& \rightarrow \text { esc }\end{aligned}$ & $\begin{array}{ll}1 & 1 \\
1 & 1\end{array}$ & $\begin{aligned} {[.] } & \rightarrow 6 \\
& \rightarrow 7 \\
& \rightarrow \mathrm{esc}\end{aligned}$ & $\begin{array}{ll}1 & 1 \\
1 & 1 \\
2 & 2\end{array}$ & $\begin{array}{l}1 / 4 \\
1 / 4 \\
2 / 4\end{array}$ & $\begin{aligned} {[.] } & \rightarrow 5 \\
& \rightarrow 6 \\
& \rightarrow 7 \\
& \rightarrow 8 \\
& \rightarrow \text { esc }\end{aligned}$ & $\begin{array}{cc}2 & 2 / 10 \\
1 & 1 / 10 \\
1 & 1 / 10 \\
2 & 2 / 10 \\
4 & 4 / 10\end{array}$ \\
\hline
\end{tabular}

Table 4. empirical value of $k_{\max }$ for some sample Images

\begin{tabular}{|l|l|l|l|l|l|l|l|}
\hline Image & BABOON & BARB & BARB2 & GOLDHILL & LENA & NOISESQUARE & PEPPERS \\
\hline Entropy & 6.002 & 4.197 & 4.790 & 4.792 & 4.522 & 5.430 & 4.514 \\
\hline$k_{\max }$ & 8 & 2 & 3 & 3 & 3 & 6 & 3 \\
\hline
\end{tabular}

As with PPM, the order of the contexts in PPAM could be variable. We can combine the $k$-approximate contexts of PPAM with the variable order-contexts of PPM. This will lead to four variations of the approach: (i) fixed order $(m)$, fixed error $(k)$; (ii) variable order $(m)$, fixed error $(k)$; (iii) fixed order $(m)$, variable error $(k)$; (iv) variable order $(m)$, variable error $(k)$. Thus, we can parameterize the PPAM model at any instant using the $(m, k)$ pair. With such an $(m, k)$-parameterization, the PPM context model can be seen as a special case of the PPAM model, with variable order contexts and exact matches $(k=0)$. The PPPM method for image coding [9] can be regarded as a PPAM model, with fixed order contexts and variable $k$, with $k=2^{n}, n=0,1,2, \ldots, \log |\Sigma|$, where $\Sigma$ is the set of distinct symbols.

The practical implementation of an efficient algorithm for $k$-approximate context search 
could be quite a challenge. Let the PPAM model be parameterized by the $(m, k)$-pair. For a given context $C=c_{m} c_{m-1} \ldots c_{1}$, we have a potential number of $k$-approximate contexts $\varphi(m, k)$ given by:

$$
\varphi(m, k)=(2 k+1)^{m}
$$

Therefore, two key questions that deserve a careful consideration are: (i) How do we efficiently search the previous contexts for $k$-approximate matches to the current context? (ii) How do we compute the conditional probability $p(s \mid C)$, given these $k$-approximate matching contexts for $C$ ? We address these problems in the following sections.

To reduce the computational requirements at the cost of a potential loss in compression, we avoid direct context search and organization at compression time. We perform the context quantization off-line, before compression begins. A major issue then will be how to approximate the conditional probability $p(s \mid C)$ needed to encode the symbol $s$ in the context $C$. We use a training set of images to pre-compute the probability tables based on a given ( $m$, k)-pair. We approximate the probability as follows:

$$
p\left(s \mid C=c_{m} c_{m-1} \ldots c_{1}\right)=\frac{p\left(s, c_{m} c_{m-1} \ldots c_{1}\right)}{p\left(C=c_{m} c_{m-1} \ldots c_{1}\right)} \cong \frac{P_{m+1}(s, C)}{P_{m}(C)}
$$

where $P_{m}$ is a table of pre-computed probabilities for order-m contexts. This eliminates the search at compression time, and requires only look-ups with the pre-computed tables. Essentially, we have transferred the time for searching to the pre-processing stage. The compression performance (and to some extent, the compression time) now depends on how closely the probability distribution of the contexts from the training set matches the conditional probability distribution from the image being compressed, and how effectively the pre-processing stage handles the huge number of contexts for natural images. Below, we briefly discuss context searching and context clustering - our solution to the problem of large number of contexts. 


\subsection{Searching For Approximate Contexts}

Though we can avoid direct search for contexts at the encoding stage, we still need an efficient method for searching for approximate contexts at the preprocessing (training) stage. In PPAM, searching for approximate contexts is performed via a tree data structure. Below, we describe this data structure and how it is used for efficient search for approximate contexts.

\subsubsection{PPAM Context Tree}

To search for approximate contexts efficiently, we store the contexts that have been previously observed in a tree structure, called the PPAM context tree. This is simply a balanced binary search tree, where each node is augmented with five parameters: $\left\langle C, i d x, \eta_{\text {occ }}, \eta_{\text {occB }}, \eta_{\text {occ }}\right\rangle$, where $C$ is the context, idx is the index of the node in a sorted order, $\eta_{\text {occ }}$ is the number of exact occurrences of the context $C, \eta_{\text {occB }}$ is the number of distinct approximate contexts to $C, \eta_{\text {occ }}$ is the total number of $k$-approximate occurrences of the context. The nodes are sorted based using a simple alphabetical listing of the contexts. Thus, the PPAM context tree is simply an AVL-tree [26], decorated with context statistics. Figure 2 shows the PPAM context tree for the example sequence used in Table I(B).

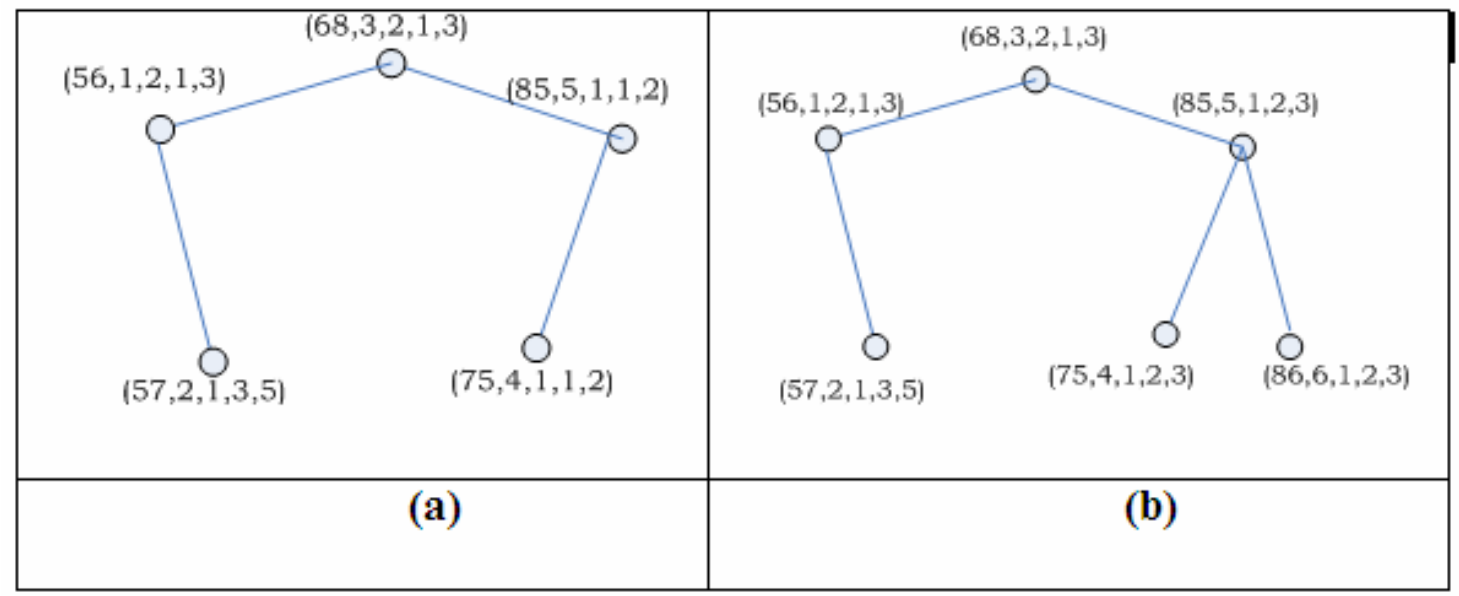

Figure 2. PPAM Context Tree. (a) Context tree for sample sequence $S=56857568$.

(b): Tree after next symbol " 6 " is appended. 
As with traditional AVL-trees, inserting a context and searching for a context each will require $O(\log u)$ time, where $u$ is the number of nodes in the tree. Since only unique contexts will be stored, we will have $u=\eta_{d},\left(\eta_{d} \leq N\right)$, where $\eta_{d}=$ number of distinct contexts, and $N=N_{1} \times N_{2}$ is the image size in pixels. Thus, for order- $m$ contexts, the overall time to construct this PPAM search tree will be in $O\left(N m \log \eta_{d}\right)$ for the entire image.

\subsubsection{Searching for $k$-approximate contexts}

Given a context $C$, we can enumerate the $\varphi(m, k)=(2 k+1)^{m}$ possible $k$-approximate contexts. The enumerated contexts can be sorted in $O(\varphi(m, k) \log \varphi(m, k))$ time. Conceptually, this enumeration and sorting can be performed as a one time process if we know $(m, k)$ and the symbol alphabet, $\Sigma$. But the number of possibilities will make this approach impractical. We avoid exhaustive enumeration and explicit sorting of the contexts, and direct search for each potential approximate context by searching for groups of contiguous contexts. This is based on the fact that, given the symbol $c_{i}$ and parameter $k$, the $k$-approximate symbols $c_{i} \pm k$ form a contiguous integer set. Therefore, considering $c_{1}$, (the symbol at the $m$-th dimension), we can see that the set of all possible $k$-approximate contexts to a given order- $m$ context $C=c_{m} c_{m-1} \ldots c_{1}$ will also be contiguous at this $m$-th dimension ${ }^{2}$. We will have a maximum of $(2 k+1)^{m-1}$ groups of such contiguous contexts. Using the previous example, at $m=2$, and $k=1$ the context $C=56$ will have nine $k$-approximate contexts: $[56]=\{45,46,47,55,56,57,65,66,67\}$. We can observe that each $(2 k+1)$ contexts starting from the first context form a contiguous set of integers, and hence will lie within nearby branches in the PPAM context tree (since they

\footnotetext{
${ }^{2}$ For ease of presentation, we use symbol $c_{m}$ as the $1^{\text {st }}$ dimension, $c_{m-1}$ as the $2^{\text {nd }}$ dimension, etc, and $c_{1}$ as the $m$-th (last) dimension.
} 
have the same prefix). By locating only the start and end points of these contiguous sets for each dimension, we can efficiently locate the $k$-approximate matches.

Based on the above, we can search for approximate contexts in the PPAM tree by performing the required logarithmic jumps one dimension at a time (rather than searching for the entire context). Consider the first dimension. Since the potential $k$-approximate contexts are implicitly sorted, they will have $2 k+1$ potential starting symbols. Since we know the starting symbol (i.e. $\left.c_{m}-k\right)$ and the end symbol $\left(c_{m}+k\right)$, for these contexts, we can search the PPAM context tree for all nodes that have their first symbol within the range: $\left[c_{m}-k, c_{m}+k\right]$. This simply requires two binary searches on the first dimension, since only the bounds are needed. The contexts within this bound will cluster within nearby regions/branches in the tree. There could be a few overlaps and outliners, and these can be handled by, say, ignoring the nodes that are not in the group, and then restructuring the tree, in a manner similar to the left-right and right-left rotations used in constructing the PPAM tree (which is a decorated AVL-tree).

The key observation is that, the set of first symbols in the potential list of approximate contexts essentially partitions the tree into two smaller trees - those contexts that have first symbols in the set (akin to marking the nodes), and those that do not. More importantly, these trees are also binary search trees, and could be balanced by appropriate re-structuring. Further, each first symbol under this set also partitions the tree to form its own binary search tree. Within this marked tree, there are potentially a maximum of $(2 k+1)^{m}$ nodes with approximate contexts to $C$, but a maximum of $(2 k+1)^{m-1}$ contiguous groups. But we have used only two $\log \eta_{d}$ binary searches to isolate them at this first dimension. Then, for each unique starting symbol in this set, we isolate its own smaller sub-tree.

We apply the above procedure on the smaller marked trees, by considering the second dimension, $c_{m-1} \pm k$, but only within the smaller sub-trees (the marked nodes). We iteratively 
apply this grouping and marking process to all the $m$-dimensions, each time focusing on the marked sub-tree. The remaining contexts in the final marked sub-trees will be the $k$ approximate contexts to the current context. At the last dimension (with symbol $c_{1}$ ), we do not need to do this for all the $(2 k+1)^{m}$ potential contexts. Rather, we consider only the starting and ending contexts for each of the $(2 k+1)^{m-1}$ subgroups, since at this last dimension, the matching $k$-approximate contexts will form contiguous subsets. Fig. 3 shows this procedure for an example context.

Using the above algorithm, we can analyze the time required to find all the $k$ approximate matches to context $C=c_{m} c_{m-1} \ldots c_{1}$. First, we need two $\log \eta_{d}$ searches to determine the start and end for all the potential approximate contexts, then $O\left(\log \eta_{d}^{j}\right)$ searches for each smaller tree at a given step of the iteration, where $\eta_{d}^{j}$ is the size of the smaller sub trees at the iteration on the $j$-th dimension. Since we need to consider only the starting and ending contexts for each of the $(2 k+1)^{m-1}$ subgroups at the last dimension, this will lead to a total time proportional to

$$
T=\log \eta_{d}+\sum_{i=0}^{m-2}(2 k+1)^{i+1} \log \eta_{d}^{m-i}+(2 k+1)^{m-1} \log \eta_{d}^{2}
$$

Clearly, the size of the sub-trees at each step of the iteration will depend on the actual symbols involved (i.e on the image being analyzed). For simplicity, however, we can assume an equiprobable distribution for the symbols. Thus, we will have:

$$
\eta_{d}^{m-i}=\eta_{d}((2 k+1) /|\Sigma|)^{i+1}
$$

Using the above, and noting that $2 k+1<<|\Sigma|$, expression (9) becomes:

$$
\begin{aligned}
T & \leq \log \eta_{d}+m \cdot(2 k+1)^{m-1} \log \left(\eta_{d}(2 k+1) /|\Sigma|\right) \\
& =O\left(\log \eta_{d}+m \cdot k^{m-1} \log \left(k \eta_{d} /|\Sigma|\right)\right)
\end{aligned}
$$

This will lead to a total of $O\left(N\left(\log \eta_{d}+m \cdot k^{m-1} \log \left(k \eta_{d} /|\Sigma|\right)\right)\right)$ time on average, for context 
searching in processing the entire image. The space complexity is in $O\left(\eta_{d} m\right)$.
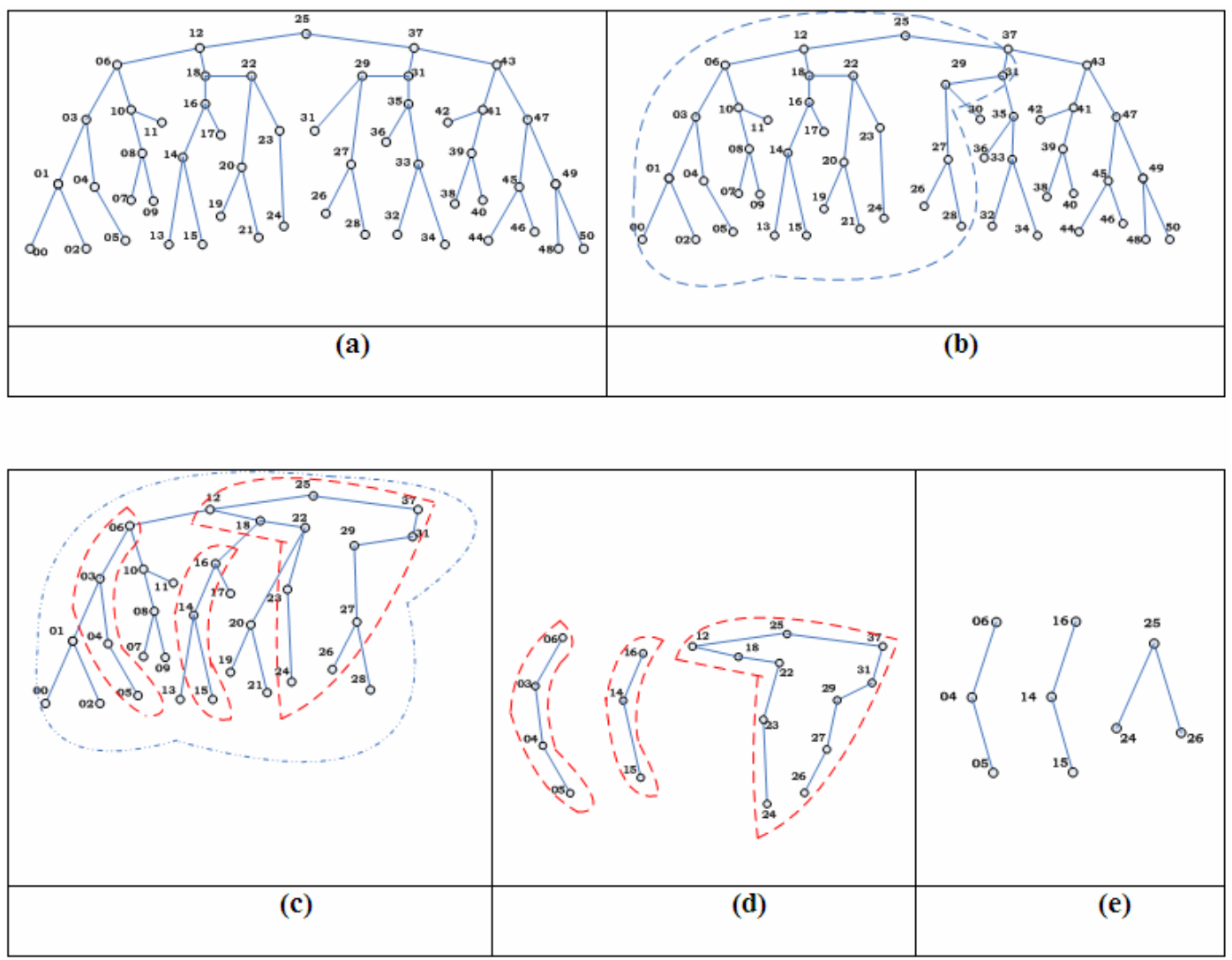

Figure 3. Searching PPAM context tree using the grouping and marking algorithm $(m=2, k=1$, current context $\left.C=c_{2} c_{1}=15\right)$. Contexts $00,01, \ldots, 50$ are assumed to have already been inserted in the tree. Potential approximate contexts $C \pm k=[15]=\{04,05,06,14,15,16,24,25,26\}$. (a): PPAM context tree for the existing contexts; (b): context tree showing the marked nodes, after first iteration (on the first dimension); (c): subtrees after second iteration; (d) independent subtrees after second iteration; (e) final pruning of independent subtrees - remaining nodes after the final pruning are the $k$-approximate contexts to $C$.

With an extra $O\left(m \eta_{d}\right)$ space, the above can be further improved by turning the $(2 k+1)^{m-1}$ factor in the algorithm into a multiplication. This will lead to an overall time in $O\left(m N \log \eta_{d}+\eta_{d} m k \log \eta_{d}\right)$ to process all pixels in the image. The first component is the time required to locate the contexts in the PPAM context tree (or to insert them at the time of first occurrence). The second component is the time needed for searching for the approximate matches to the contexts. The overall space complexity still remains the same, even with the extra space. 
In general, $m$ and $k$ are very small relative to $|\Sigma|, N$ (and hence $\eta_{d}$ ). For instance, we have $|\Sigma|=511$ for prediction errors from an 8 -bit image, $m=4$ or $m=5$, and $k \leq 8$. For LENNA, image size is $N=512 \times 512$, or 262144 pixels, and $\eta_{d}=115,125$. Thus, overall, the above algorithm will default to $O\left(N \log \eta_{d}\right)$ time for both constructing the PPAM context tree and for searching for approximate contexts. The above analysis is with respect to a single $k$ value. However, the overall complexity remains the same, since $k_{\max }$ is a small constant, relative to the image size.

\subsection{Context Clustering}

Fig. 4 shows the distribution of $k$-approximate contexts for a sample image. Contexts are sorted based on their frequency of occurrence. We observe that for natural images, the number of high order contexts could be quite large. This leads to a time consuming process for context searching and requires a considerable amount of memory for storage. Expectedly, the number of contexts (and the attendant complexity) increases with increasing value of $k$, the error parameter. While the approximate contexts mitigate the problem of context sparsity in images, we also have to consider the important issue of model cost [27]. Without a careful consideration, the huge number of contexts could lead to a loss in coding efficiency. To further reduce the model cost as well as space and time requirements, we quantize (cluster) the contexts based on their similarity. Without quantization, we could need a potentialo $o\left(|\Sigma|^{m}\right)$ or $O\left(|\Sigma|^{m+1}\right)$ entries in tables $P_{m}$ and $P_{m+1}$ respectively. This could be potentially problematic, given that $|\Sigma|=511$ for prediction error symbols from 8-bit gray-scale images.

The context quantization problem is analyzed in [28] in a way similar to the vector quantization problem. The relative entropy is used as a distortion measure and a Lloyd-style 
algorithm is applied for context quantization in order to minimize the distortion. However, this approach is not only time consuming, but also requires a huge memory to store the quantization tables. In this paper, we introduce a simple quantization scheme to avoid the huge time and space requirements. We use a template of neighboring prediction residues for our contexts (see in Figure 5). Here the $c_{i}{ }^{\prime}$ s represent the neighboring symbols used for the construction of PPAM contexts.

In PPAM, we group the contexts based on their distance from a reference context (for example, context $C=0000$, for order- 4 contexts). Specifically, we use the square of Euclidian distance (SED) for the contexts. For an order-m context $C=c_{m} c_{m-1} \ldots c_{1}$, the SED is defined as follows:

$$
\operatorname{SED}(C)=c_{m}^{2}+c_{m-1}^{2}+\ldots+c_{1}^{2}
$$

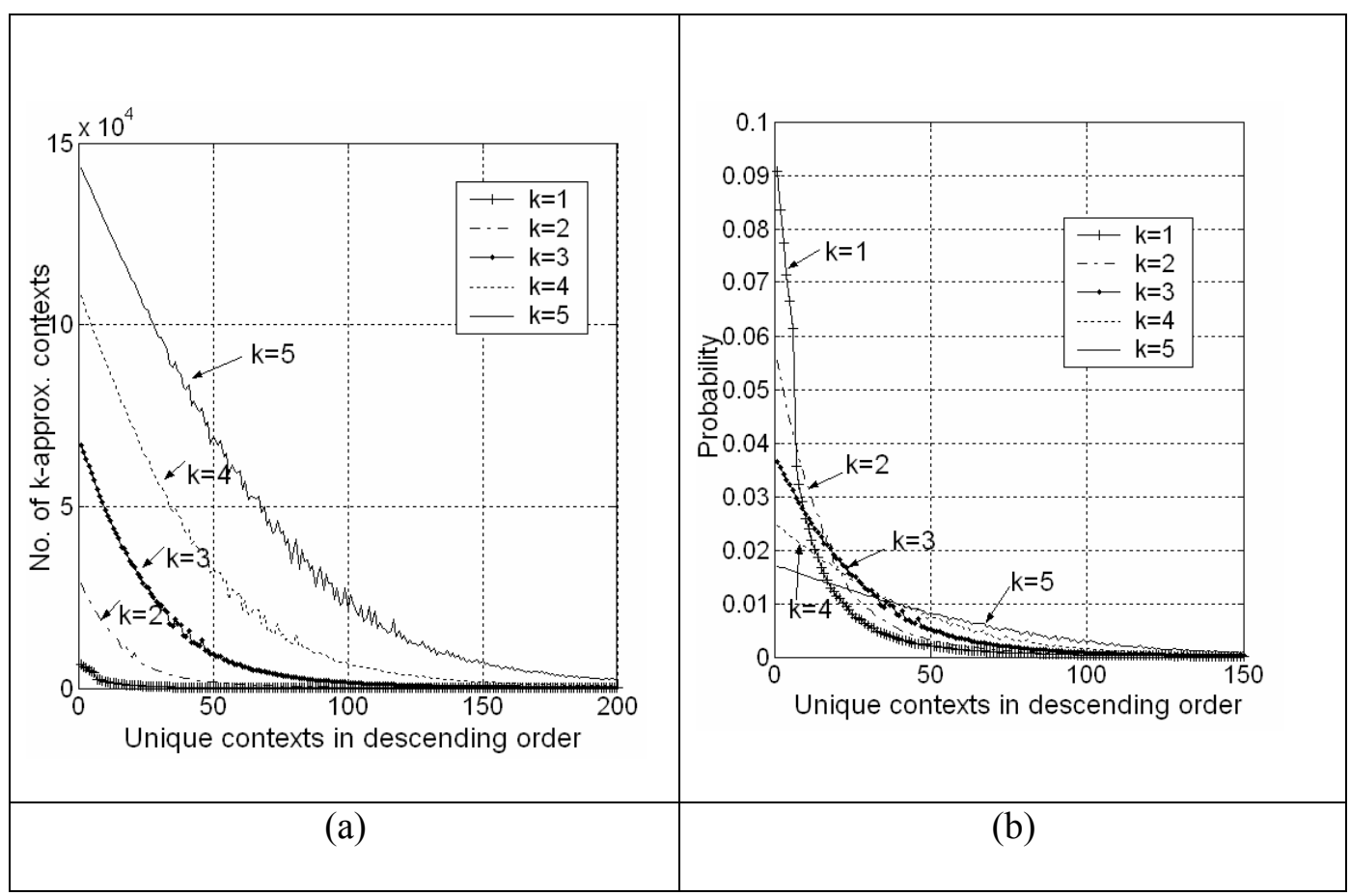

Figure 4. Distribution of $k$-approximate contexts for LENNAGREY; (a) approximate context counts; (b) normalized counts. These provide the conditioning distributions used for coding the symbols. 


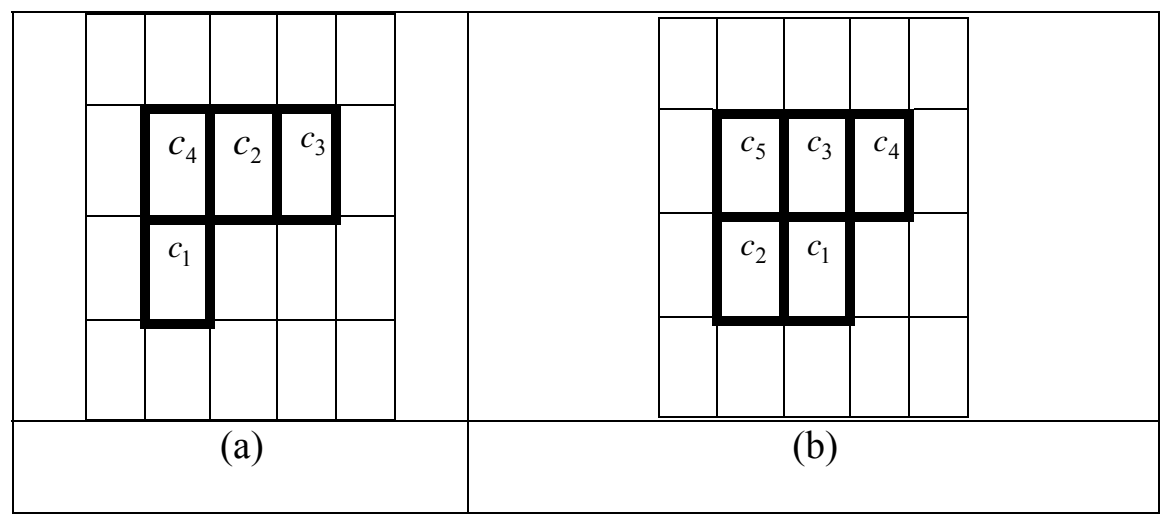

Figure 5. Contexts template for PPAM (a) order-4 context (b) order-5 context

The SED value is a non-negative integer and thus can be used as an index for that context. Contexts with the same SED values are then grouped into the same partition, denoted as $Q_{k}^{m}$ for order- $m$ contexts with approximation parameter $k$. Since contexts in the same partition have the same distance from the reference point, the SED value, $\operatorname{SED}(C)$ also forms an index for the group, $Q_{k}^{m}$. Essentially, this partitioning scheme groups all contexts that appear on the surface of an $m$-dimensional hypersphere into the same cluster. Then for a given partition, we estimate a probability distribution for the symbols.

There are several ways to obtain this estimation. For instance, we can use the average probability of all the contexts in the partition, as was done in [28]:

$$
p\left(s \mid Q_{k}^{m}\right)=\frac{1}{\sum_{C_{i} \in Q_{k}^{m}} p\left(C_{i}\right)} \sum_{C_{i} \in Q_{k}^{m}} p\left(C_{i}\right) p\left(s \mid C_{i}\right)
$$

where $p\left(C_{i}\right)$ is the estimated probability for the context $C_{i}$, and $p\left(s \mid C_{i}\right)$ is the estimated conditional probability for symbol $s$ in the context $C_{i}$. For improved efficiency, in PPAM, we use a different method to construct the conditional probability distribution for each partition $Q_{k}^{m}$. Since all the contexts $C_{i}$ in the group $Q_{k}^{m}$ have been quantized into partition $Q_{k}^{m}$, we use the maximum of the $p\left(C_{i}\right)$ 's as the probability for the given partition $Q_{k}^{m}$, We then use $p\left(Q_{k}^{m}\right)$ as the probability of any context $C_{i}, \forall C_{i} \in Q_{k}^{m}$ : 


$$
p\left(C_{i}\right)=p\left(Q_{k}^{m}\right)=\max _{C_{i} \in Q_{k}^{m}}\left\{p\left(C_{i}\right)\right\}
$$

To determine the conditional probability for a given symbol $s$ in the context $C_{i}$, we consider $\left(s, C_{i}\right)$ as an order- $(m+1)$ context, as shown in Fig.4 (b). All the possible order- $(m+1)$ contexts are also quantized into partitions $Q_{k}^{m+1}$ based on the square of their Euclidian distances. A probability value $p\left(Q_{k}^{m+1}\right)$ is assigned to each partition as described above using (15), with $C_{i} \in Q_{k}^{m+1}$. Let $\Sigma$ be the symbol alphabet. (Thus, $s \in \Sigma=\{-255,-254, \cdots,-1,0,1, \cdots, 254,255\}$ for prediction errors from an 8-bit image). Then the conditional probability distribution of $s$ in the context $C_{i}$ is given by:

$$
p\left(s \mid C_{i}\right)=\frac{P_{m+1}\left(s, C_{i}\right)}{P_{m+1}\left(C_{i}\right)}=\frac{1}{A^{\prime}} \frac{p\left(Q_{k}^{m+1}\right)}{p\left(Q_{k}^{m}\right)}
$$

where $s \in \Sigma, C_{i} \in Q_{k}^{m},\left(s, C_{i}\right) \in Q_{k}^{m+1}$, and $A^{\prime}$ is a constant used to normalize the probabilities. We further observe that the denominator $p\left(Q_{k}^{m}\right)$ is always a constant for a given context $C_{i}$ (see (14) ), thus it can be combined with $A^{\prime}$ for an even simpler formulation:

$$
p\left(s \mid C_{i}\right)=\frac{1}{A} p\left(Q_{k}^{m+1}\right)
$$

where $A$ is a combined constant.

This quantization scheme may not be optimal in the sense of relative entropy (see for instance [29] ). However, since the SED value of a context can be used as the index value to the corresponding quantization partition. This simple method avoids assigning each context to a partition iteratively and does not require a large storage for the quantization tables. Eq. (16) shows that to construct the conditional probability distribution, we only need to store one probability value for each quantization partition.

Table 5 shows an example context quantization in PPAM. The contexts have a fixed order of 3. Each of the quantization results consists of an SED value and some statistics (number of occurrences of the context) which is the maximum statistics of that partition. The 
total number of partitions might be very large for an image. For instance, for an order-5 context $C=c_{5} c_{4} c_{3} c_{2} c_{1}$, with $\max \left\{c_{i}\right\}=255$, the maximum SED value will be 325,125 . We reduce the number of partitions based on the skewed nature of the prediction residues (used to form the contexts). Most prediction errors outside the range $[-50,50]$ are unlikely to occur frequently. Thus, we can use a significantly fewer quantization partitions (e.g. 1000 to 5000) without adverse impact on the compression performance. Contexts whose SED values do not fall into any of the partitions are assigned the probability of the partition with the largest SED value.

Table 5. EXAMPLE CONTEXT QUANTIZATION IN PPAM

\begin{tabular}{|c|c|c|c|c|}
\hline Index & Context & SED & \# Occurrence & Quantization \\
\hline 0 & 000 & 0 & 100 & $(0,100)$ \\
\hline \multirow[t]{3}{*}{1} & 001 & 1 & 50 & \multirow[t]{3}{*}{$(1,70)$} \\
\hline & 010 & 1 & 70 & \\
\hline & 100 & 1 & 12 & \\
\hline \multirow[t]{3}{*}{2} & 011 & 2 & 35 & \multirow[t]{3}{*}{$(2,54)$} \\
\hline & 101 & 2 & 33 & \\
\hline & 110 & 2 & 54 & \\
\hline 3 & 111 & 3 & 19 & $(3,19)$ \\
\hline \multirow[t]{3}{*}{4} & 002 & 4 & 11 & \multirow[t]{3}{*}{$(4,67)$} \\
\hline & 020 & 4 & 67 & \\
\hline & 200 & 4 & 32 & \\
\hline
\end{tabular}

\subsection{PPAM Implementation}

Figure 6 shows a block diagram for PPAM-based image compression. Compression using PPAM involves four basic steps: preprocessing, prediction, context modeling, and arithmetic coding.

The preprocessing is an off-line training process used to estimate the true conditional probability distributions from a input image (see Figure 7). Important context statistics are obtained during the search process based on the $k$-approximate matches described previously. All the contexts are then quantized and probability distributions are established which are then used at both the encoder and decoder. The preprocessing is performed off-line before any compression. 


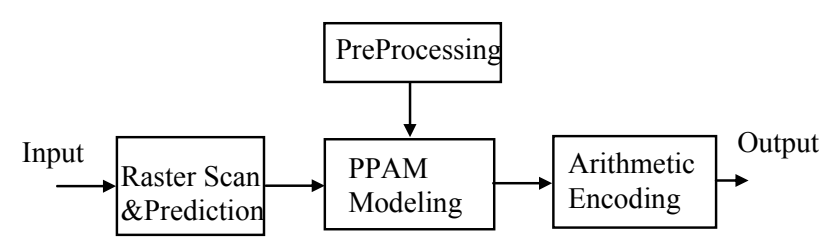

Figure 6. PPAM Lossless Image Compression Scheme

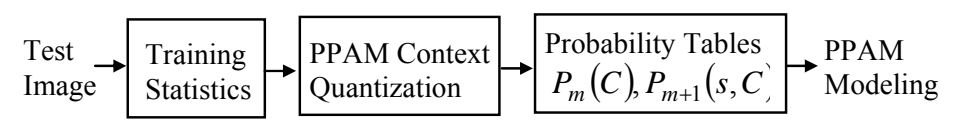

Figure 7. PPAM Preprocessing

\subsubsection{Image Grouping - Modeling Context Statistics}

The encoder can compute the statistics of approximate contexts, but it needs to communicate same to the decoder for lossless decoding. Given the number of entries in the table (10005000), sending the entire conditional probability distributions at each step of the compression will defeat the purpose of compression. We solve this problem using image grouping. All calculations here are based on the prediction errors.

One major advantage of using off-line preprocessing is that the constructed probability distributions can be used for encoding other images that are similar to the corresponding test image. We divide the training images into groups, and thus rather than performing the preprocessing for each input image, we perform the process for only selected group representatives. We use a set of training images to construct the probability tables, one for each image class. The statistics for a group are then used to compress each image in the group. This offline pre-computation means that we save time in encoding and decoding. 
To avoid the difficult problem of robust image classification (see [30]), given an input image, we group it based on its relative entropy with the group representatives, and the difference in their standard deviations. The relative entropy (also called Kullback-Leibler $(K L)$ divergence) is defined as follows [31]:

$$
K L(p, q)=\sum_{x} p(x) \log \left(\frac{p(x)}{q(x)}\right)
$$

where, $p$ and $q$ are two probability distributions. Since the $K L$-divergence is not symmetric, we consider the symmetric form:

$$
K L S(p, q)=\frac{1}{2}\left(\sum_{x} p(x) \log \left(\frac{p(x)}{q(x)}\right)+\sum_{x} q(x) \log \left(\frac{q(x)}{p(x)}\right)\right)
$$

Given two images represented by their prediction errors $P$, and $Q$, we then define the distance measure between them as follows:

$$
D(P, Q)=\omega_{1} K L S(p, q)+\left(1-\omega_{1}\right)\left(\sigma_{P}-\sigma_{Q}\right), \quad 0 \leq \omega_{1} \leq 1
$$

where, $p$ and $q$ are the respective probability distributions for $P$ and $Q, \sigma_{P}$ and $\sigma_{Q}$ are their respective standard deviations, and $\omega_{1}$ is a are weight. Thus, for a given image $I$, we first classify it into group $G_{k}$, and then compress it with the statistics from the group representative image $I_{k}$, where

$$
k=\arg \min _{j}\left\{D\left(I, G_{j}\right)\right\} .
$$

Apart from speed and memory efficiency, this classification-based approach has potential applications in compressing image sets, where the images have similar characteristics, An example is in high throughput imaging screening applications [32], where thousands of fluorescence microscopy images are generated (for the same experiment using the same imaging facility) in order to study complex biological processes. 


\subsubsection{Prediction and Range Selection}

In lossless image compression, pixel prediction is often performed before entropy coding. Thus, the overall performance of an image coding scheme will be significantly influenced by the prediction algorithm used. In this work, however, we focus mainly on context modeling, and hence we use existing prediction schemes. We consider EDP - edge directed prediction [33], and MED - median edge detector[11] used in JPEG-LS.

Let $I(x, y)=$ pixel value at position $(x, y)$ in the image, $P(x, y)=$ corresponding prediction value from the predictor, and $E(x, y)=I(x, y)-P(x, y)$ is the prediction residue. Encoding is applied on $E$ rather than $I$ since the distribution of $E$ is more suitable for compression. Context searching and quantization are also applied on the prediction errors rather than the pixel values. Since the range of $I$ is $[0,255]$ for 8-bit grey-scale images, the possible range of $E$ will be $[-255,255]$, for a total of 511 possible symbols. We can improve compression by reducing the possible number of coding symbols. Since $I$ is within the range $[0,255]$, we can reduce the range of $E$ from $[-255,255]$ to $[-P, 255-P]$. That is, given the prediction value $P$, the total number of possible values for $E$ will be 256 instead of 511 . By examining $E$ for the test images, we found that most of them fall into a relatively small range. For example, $99 \%$ of the error values $E$ for the image BALLOON are in the range $[-5,5]$ after EDP prediction. Thus we use a selectable range to reduce the number of symbols needed to code the prediction error $E$ :

$$
\text { Range }= \begin{cases}{[-R, R]} & \text { if } R<P<255-R \\ {[-P, R]} & \text { if } P<R \wedge P<255-R \\ {[-R, 255-P]} & \text { if } P>R \wedge P>255-R\end{cases}
$$

where $R$ represents the estimated range $[-R, R]$ for the prediction error $E$. If an error value falls outside the range defined by (20), then an escape symbol (Esc) is sent to the compressed stream and the error value is compressed based on a new range given by: 


$$
\text { Range }^{*}= \begin{cases}{[-P,-R-1] \cup[R+1,255-P]} & \text { if } R<P<255-R \\ {[R+1,255-P]} & \text { if } P<R \wedge P<255-R \\ {[-P,-R-1]} & \text { if } P>R \wedge P>255-R\end{cases}
$$

Different images may require different ranges for best performance. To maximize compression, $R$ can be estimated based on the residue image, and then stored as part of the compressed bit stream. In the current implementation, we use the value of $R$ such that about $99 \%$ of the error values fall into the range $[-R, R]$. Applying the range selection scheme resulted in about $1 \%-2 \%$ improvement in compression performance.

\subsection{Results}

\subsubsection{Experimental Setup}

We tested the performance of the proposed PPAM using publicly available standard test images (Images were obtained from: the USC-SIPI image database http://decsai.ugr.es/cvg/CG/base.htm; the TMW website, http://www.csse.monash.edu.au/ bmeyer/tmw/, and from http://sipi.usc.edu/database/). We use PPAM1 to denote PPAM using EDP as the prediction scheme, while PPAM2 denotes PPAM using MED as the prediction scheme. Experiments were performed in MATLAB 7 and Microsoft Visual Studio 6.0 environment, running on a Pentium IV, 2.8GHz PC with $512 \mathrm{MB}$ of RAM. All compression results are reported in bits per pixel (bpp), unless otherwise stated. We also test the PPAM on neurite images[32]. The neurite images were obtained from Professor Yuan's Lab at the Department of Cell Biology, Harvard Medical School. The images are acquired using multi-channel automated florescence microscopy. Since the neurite images have common features (for example, neurite structures), only one neurite image from the same experiment need to be used for the PPAM offline training and then the obtained context model can be used to compress all of the other images. 


\subsubsection{PPAM Coding Parameters and Training}

Table 6 and Table 7 show the PPAM algorithmic parameters used for the training images. The parameters considered are the range values $R$, the coverage for the range, the escape symbol, Esc, and the approximation parameter $k$. We can notice that for the same image, the best results for the two prediction schemes typically require different sets of parameters. Values for $R$ and EsC are determined experimentally. However, once determined, these parameters are fixed and used for any other image in the same image group.

Table 8 shows the coding results for different images, using different values of $k$ at the pre-processing stage, using PPAM1. Expectedly, the coding results vary with $k$. Performance improves with increasing $k$ until $k$ reaches a maximum value, $k_{\max }$. Performance then starts to decrease, with further increase in $k$ beyond $k_{\max }$. In particular, we notice that best results are usually obtained at $k>0$. This clearly shows the importance of the proposed $k$-approximate modeling. $k_{\max }$ tends to increase with increasing entropy of the image.

Table 6. PPAM1 CODING PARAMETERS

\begin{tabular}{|l|l|l|l|l|l|l|l|l|l|}
\hline Image & GroupID & Source & Entropy & STD & $\boldsymbol{k}$ & Range(R) & R-coverage & EsC & PPAM1 \\
\hline BABOON1 & 7 & $\# 47$ & 6.007 & 17.257 & 8 & 105 & $99.95 \%$ & 5 & 5.829 \\
\hline CROWD & 6 & $\# 43$ & 4.266 & 6.863 & 2 & 31 & $99.81 \%$ & 80 & 4.082 \\
\hline GOLDHILL & 3 & $\# 2$ & 4.797 & 7.483 & 3 & 53 & $99.97 \%$ & 4 & 4.751 \\
\hline LENA & 2 & TMW & 4.522 & 6.213 & 3 & 34 & $99.96 \%$ & 30 & 4.428 \\
\hline LENNAGREY & 1 & TMW & 4.159 & 4.970 & 2 & 5 & $99.98 \%$ & 30 & 4.053 \\
\hline RIVER & 4 & $\# 45$ & 5.077 & 9.503 & 3 & 36 & $99.12 \%$ & 100 & 4.895 \\
\hline RIVER2 & 5 & $\# 30$ & 5.285 & 10.756 & 3 & 42 & $99.53 \%$ & 100 & 5.135 \\
\hline
\end{tabular}

Table 7. PPAM2 CODING PARAMETERS

\begin{tabular}{|l|l|l|l|l|l|l|l|l|l|}
\hline Image & GroupID & Source & Entropy & STD & $\boldsymbol{k}$ & Range $(\boldsymbol{R})$ & R-coverage & EsC & PPAM2 \\
\hline BABOON1 & 6 & $\# 47$ & 6.275 & 20.842 & 6 & 50 & $99.90 \%$ & 80 & 6.057 \\
\hline BARB & 7 & $\# 1$ & 5.204 & 12.802 & 2 & 50 & $99.40 \%$ & 100 & 4.857 \\
\hline BIRD & 3 & $\# 26$ & 3.130 & 7.092 & 0 & 14 & $99.37 \%$ & 800 & 3.738 \\
\hline BRIDGE & 2 & $\# 25$ & 2.704 & 6.025 & 0 & 14 & $99.87 \%$ & 600 & 2.966 \\
\hline LENA & 8 & TMW & 4.892 & 8.660 & 3 & 50 & $99.92 \%$ & 40 & 4.757 \\
\hline LENNAGREY & 9 & TMW & 4.547 & 7.134 & 2 & 38 & $99.88 \%$ & 50 & 4.399 \\
\hline CROWD & 4 & $\# 43$ & 3.630 & 8.025 & 0 & 18 & $99.66 \%$ & 800 & 3.562 \\
\hline MOUNTAIN & 1 & $\# 40$ & 3.523 & 4.940 & 0 & 12 & $99.91 \%$ & 350 & 3.785 \\
\hline RIVER2 & 5 & $\# 30$ & 4.425 & 11.794 & 2 & 32 & $99.67 \%$ & 300 & 4.884 \\
\hline
\end{tabular}

Note: the "source" indicates where we obtained the test images. "\#" indicates the corresponding image number at the website (http://decsai.ugr.es/cvg/CG/base.htm); "TMW" denotes the TMW website. This applies to all the following tables. 
Table 8. EFFECT OF VARYING $k$ FOR DIFFERENT IMAGES

\begin{tabular}{|l|l|l|l|l|l|}
\hline $\boldsymbol{k}$ & LENA & $\boldsymbol{k}$ & BABOON & $\boldsymbol{k}$ & BARB \\
\hline 0 & 4.697 & 0 & 6.031 & 0 & 4.320 \\
\hline 1 & 4.581 & 4 & 5.887 & 1 & 4.116 \\
\hline 2 & 4.451 & 6 & 5.828 & $\mathbf{2}$ & $\mathbf{4 . 0 6 9}$ \\
\hline $\mathbf{3}$ & $\mathbf{4 . 4 2 8}$ & $\mathbf{8}$ & $\mathbf{5 . 8 2 5}$ & 3 & 4.095 \\
\hline 4 & 4.439 & 10 & 5.849 & 4 & 4.163 \\
\hline 5 & 4.473 & 12 & 5.891 & 5 & 4.262 \\
\hline
\end{tabular}

\subsubsection{Comparative Results on Test Images}

We compare the performance of PPAM with other state-of-the-art compression schemes. Since PPAM focus on context modeling, it is important to isolate the effect of the prediction scheme on compression performance. Thus, for each given predictor (EDP or MED), we compare our results with published compression result obtained using the same prediction scheme. For EDP, this means comparing with the EDP codec $[33]^{3}$. For MED, this means comparing with JPEG-LS [11]. Essentially, this means we are comparing performance of PPAM with that of the context model used in the two popular schemes. The PPAM results reported in this section are based on image grouping. Since different prediction schemes could lead to different values for entropy and standard deviation, the image grouping could be different for the two predictors. The group representatives are chosen to be the image whose standard-deviation and entropy are closest to the group centroid. The results are shown in Tables 9. We note that the results reported in this subsection (comparative performance) are compression results for test images. That is, results obtained using PPAM contexts as defined by the respective group representative to compress images in a group. The results show that, in almost all cases considered, PPAM1 performs better than EDP on the same image (both use the same EDP prediction errors), and PPAM2 performs better than JPEG-LS on the same image (both use the same MED prediction errors). The table also compares PPAM results those from other popular compression schemes, such as CALIC

\footnotetext{
${ }^{3}$ EDP results are based on the EDP codec available at: http://csee.wvu.edu/ xinl/software.html .
} 
(using arithmetic encoder) and JPEG2000. The results show that the proposed PPAM algorithm is quite competitive when compared with other state-of-the-art algorithms.

Table 10 shows the compression performance of PPAM when compared with CALIC (using arithmetic encoder), EDP, JPEG-LS, and JPEG2000. The results show that the proposed PPAM with MED outperforms all of the other methods on the test images.

Table 11 shows the comparative compression performance of PPAM on the neurite images, when compared with CALIC (using arithmetic encoder), EDP, JPEG-LS, and JPEG2000. The results show that the proposed PPAM outperforms all of the other methods on this image modality, with $5 \%$ improvement over CALIC. This shows the significance of context clustering and appropriate image grouping in PPAM. Neurite02 is used as the representative image for the group. The parameters for PPAM+EDP were $\mathrm{k}=1, \mathrm{ESC}=12$, Range $=10$ and for PPAM+MED: $\mathrm{k}=0, \mathrm{ESC}=400$, Range $=6$.

Table 9. COMPARATIVE RESULTS WITH EDP AND JPEG-LS (BITS PER PIXEL)

\begin{tabular}{|c|c|c|c|c|c|c|c|c|c|}
\hline \multicolumn{2}{|c|}{ Group ID } & \multirow[t]{2}{*}{ Image } & \multirow[t]{2}{*}{ source } & \multirow[t]{2}{*}{ Entropy } & \multirow[t]{2}{*}{ PPAM1 } & \multirow[t]{2}{*}{ EDP } & \multirow[t]{2}{*}{ PPAM2 } & \multirow[t]{2}{*}{ JPEG-LS } & \multirow[t]{2}{*}{ JPEG2000 } \\
\hline PPAM1 & PPAM2 & & & & & & & & \\
\hline $7 *$ & $6^{*}$ & Baboon1 & $\# 47$ & 7.358 & 5.829 & 5.974 & 6.057 & 6.036 & 6.147 \\
\hline 2 & 4 & Baboon2 & $\# 5$ & 4.462 & 4.559 & 4.585 & 4.072 & 4.201 & 4.757 \\
\hline 1 & $7 *$ & Barb & $\# 1$ & 7.555 & 4.107 & 4.340 & 4.857 & 4.691 & 4.639 \\
\hline 2 & $3^{*}$ & Bird & $\# 26$ & 5.430 & 4.453 & 4.427 & 3.896 & 3.984 & 4.585 \\
\hline 1 & 1 & Parrot & USC & 6.178 & 3.544 & 3.565 & 3.245 & 3.480 & 3.695 \\
\hline 2 & 4 & Boat & $\# 48$ & 6.132 & 4.330 & 4.358 & 4.010 & 4.271 & 4.465 \\
\hline $6^{*}$ & $2 *$ & Bridge & $\# 25$ & 5.088 & 3.737 & 3.641 & 2.966 & 3.100 & 3.750 \\
\hline 3 & 4 & Car & $\# 13$ & 6.251 & 4.270 & 4.196 & 3.797 & 4.068 & 4.285 \\
\hline $7 *$ & 4 & Clock & USC & 5.716 & 3.708 & 3.655 & 3.279 & 3.522 & 3.874 \\
\hline 3 & 4 & Couple & $\# 41$ & 6.359 & 4.735 & 4.772 & 4.497 & 4.698 & 4.903 \\
\hline $8 *$ & $4^{*}$ & Crowd & $\# 43$ & 5.927 & 4.082 & 4.100 & 3.562 & 3.924 & 4.288 \\
\hline $3 *$ & 4 & goldhill & $\# 2$ & 6.479 & 4.752 & 4.791 & 4.478 & 4.732 & 4.892 \\
\hline 5 & 5 & House & $\# 36$ & 5.638 & 5.290 & 5.272 & 5.044 & 5.138 & 5.435 \\
\hline $2 *$ & 8* & lena & TMW & 7.594 & 4.428 & 4.576 & 4.757 & 4.607 & 4.718 \\
\hline $1^{*}$ & $9 *$ & lennagrey & TMW & 7.447 & 4.053 & 4.215 & 4.399 & 4.238 & 4.336 \\
\hline 5 & 5 & man & $\# 12$ & 6.370 & 4.985 & 5.038 & 4.891 & 4.928 & 5.218 \\
\hline 1 & $1 *$ & Mountain & $\# 40$ & 5.757 & 4.112 & 4.207 & 4.135 & 4.135 & 4.277 \\
\hline 4 & 5 & Plant & USC & 5.118 & 5.040 & 5.129 & 5.224 & 5.176 & 5.343 \\
\hline $4^{*}$ & 5 & River & $\# 45$ & 6.459 & 4.895 & 5.015 & 4.916 & 4.925 & 5.143 \\
\hline $5^{*}$ & $5 *$ & River2 & $\# 30$ & 5.907 & 5.135 & 5.202 & 4.884 & 4.970 & 5.364 \\
\hline 5 & 5 & tree & $\# 37$ & 5.535 & 5.373 & 5.441 & 5.146 & 5.271 & 5.520 \\
\hline 1 & 1 & washsat & USC & 4.384 & 4.382 & 4.389 & 4.057 & 4.131 & 4.485 \\
\hline 5 & 5 & waterwheel & $\# 33$ & 5.948 & 5.152 & 5.178 & 4.914 & 4.963 & 5.300 \\
\hline 1 & 1 & zelda & TMW & 6.267 & 3.839 & 4.030 & 3.746 & 4.029 & 4.054 \\
\hline \multicolumn{2}{|c|}{ Average } & & & 6.057 & 4.533 & 4.587 & 4.368 & 4.467 & 4.728 \\
\hline
\end{tabular}


Table 10. COMPARATIVE RESULTS (IN BPP) WITH OTHER STANDARD IMAGE COMPRESSION ALGORITHMS. BEST RESULTS ARE INDICATED IN BOLD FOR EACH IMAGE.

\begin{tabular}{|c|c|c|c|c|c|c|c|c|}
\hline Image & Source & Entropy & EDP & PPAM1 & JPEG-LS & PPAM2 & CALIC & JPEG 2000 \\
\hline Boat & $\# 48$ & 6.132 & 4.358 & 4.33 & 4.271 & $\mathbf{4 . 0 1}$ & 4.181 & 4.465 \\
\hline Car & $\# 13$ & 6.251 & 4.196 & 4.270 & 4.068 & $\mathbf{3 . 7 9 7}$ & 3.946 & 4.285 \\
\hline Couple & $\# 41$ & 6.359 & 4.772 & 4.735 & 4.698 & $\mathbf{4 . 4 9 7}$ & 4.618 & 4.903 \\
\hline House & $\# 36$ & 5.638 & 5.272 & 5.290 & 5.138 & 5.044 & $\mathbf{4 . 9 8 3}$ & 5.435 \\
\hline Man & $\# 12$ & 6.370 & 5.038 & 4.985 & 4.928 & 4.891 & $\mathbf{4 . 8 0 8}$ & 5.218 \\
\hline Parrot & USC & 6.178 & 3.565 & 3.544 & 3.480 & $\mathbf{3 . 2 4 5}$ & 3.327 & 3.695 \\
\hline Plant & USC & 5.118 & 5.129 & $\mathbf{5 . 0 4 0}$ & 5.176 & 5.224 & 5.108 & 5.343 \\
\hline Tree & $\# 37$ & 5.535 & 5.441 & 5.373 & 5.271 & 5.146 & $\mathbf{5 . 1 4 1}$ & 5.520 \\
\hline Waterwheel & $\# 33$ & 5.948 & 5.178 & 5.152 & 4.963 & 4.914 & $\mathbf{4 . 7 9 3}$ & 5.300 \\
\hline Zelda & TMW & 6.267 & 4.030 & 3.839 & 4.029 & $\mathbf{3 . 7 4 6}$ & 3.908 & 4.054 \\
\hline Average & & $\mathbf{5 . 9 8 0}$ & $\mathbf{4 . 6 9 8}$ & $\mathbf{4 . 6 5 6}$ & $\mathbf{4 . 6 0 2}$ & $\mathbf{4 . 4 5 1}$ & $\mathbf{4 . 4 8 1}$ & $\mathbf{4 . 8 2 2}$ \\
\hline
\end{tabular}

Table 11. COMPARATIVE RESULTS (IN BPP) WITH OTHER STANDARD COMPRESSION ALGORITHMS ON NEURITE IMAGES. THE REPRESENTATIVE IMAGE FOR PPAM WAS NEURITE02, DENOTED WITH “*”

\begin{tabular}{|c|c|c|c|c|c|c|c|}
\hline Images & Entropy & EDP & PPAM1 & PPAM2 & CALIC & JPEG-LS & JPEG2000 \\
\hline Neurite01 & 3.394 & 3.259 & 2.937 & $\mathbf{2 . 7 9 1}$ & 2.932 & 3.101 & 3.118 \\
\hline Neurite02* & 3.460 & 3.503 & 3.279 & $\mathbf{2 . 9 0 2}$ & 3.299 & 3.400 & 3.445 \\
\hline Neurite03 & 3.181 & 3.028 & 2.803 & 2.789 & $\mathbf{2 . 6 5 8}$ & 2.906 & 2.913 \\
\hline Neurite04 & 3.050 & 2.946 & 2.778 & 2.694 & $\mathbf{2 . 5 6 1}$ & 2.839 & 2.889 \\
\hline Neurite05 & 3.768 & 3.822 & 3.682 & $\mathbf{3 . 3 7 2}$ & 3.702 & 3.811 & 3.790 \\
\hline Neurite06 & 3.299 & 3.210 & 2.956 & $\mathbf{2 . 8 1 1}$ & 2.950 & 3.106 & 3.114 \\
\hline Neurite07 & 3.637 & 3.672 & 3.498 & $\mathbf{3 . 1 3 4}$ & 3.553 & 3.661 & 3.627 \\
\hline Neurite08 & 3.346 & 3.339 & 3.123 & $\mathbf{2 . 8 9 6}$ & 3.184 & 3.288 & 3.282 \\
\hline Neurite09 & 3.158 & 3.104 & 2.911 & $\mathbf{2 . 7 7 2}$ & 2.770 & 3.008 & 3.035 \\
\hline Neurite10 & 3.212 & 3.111 & 2.931 & 2.800 & $\mathbf{2 . 7 6 8}$ & 3.013 & 3.016 \\
\hline Average & $\mathbf{3 . 3 5 1}$ & $\mathbf{3 . 2 9 9}$ & $\mathbf{3 . 0 9 0}$ & $\mathbf{2 . 8 9 6}$ & $\mathbf{3 . 0 3 8}$ & $\mathbf{3 . 2 1 3}$ & $\mathbf{3 . 2 2 3}$ \\
\hline
\end{tabular}

\subsubsection{Encoding and Decoding Time}

In terms of computational time, PPAM1 and PPAM2 on-line compression has time requirements similar to CALIC and EDP. Table 12 lists the running time in seconds for PPAM1 and PPAM2 for some of the test images with different sizes and entropies. These running times include prediction, PPAM modeling, and entropy encoding. This does not include the offline training stage, which is a one time process, performed only on the training images (the group representatives). At compression, this does not play any role in determining the compression time for the test images. For the off-line PPAM preprocessing, the running time varies depending on the size and the entropy of the input image. 
Table 12. ENCODING AND DECODING TIME FOR PPAM1 AND PPAM2 (IN SECONDS)

\begin{tabular}{|c|c|c|c|c|c|c|}
\hline Image & Size & Entrop & \multicolumn{2}{|c|}{ PPAM1(sec) } & \multicolumn{2}{c|}{ PPAM2(sec) } \\
\cline { 4 - 7 } & & $\mathbf{y}$ & Encode & Decode & Encode & Decode \\
\hline Baboon1 & $512 \times 512$ & 7.358 & 25.5 & 24.8 & 16.5 & 15.3 \\
\hline Baboon1* & $256 \times 256$ & 7.063 & 2.7 & 2.6 & 1.9 & 1.8 \\
\hline Bird & $512 \times 512$ & 5.430 & 18.2 & 18.1 & 10.3 & 9.5 \\
\hline Bird* & $256 \times 256$ & 5.267 & 1.8 & 1.8 & 0.9 & 1.0 \\
\hline Couple & $512 \times 512$ & 6.359 & 22.9 & 21.7 & 12.4 & 11.8 \\
\hline Couple* & $256 \times 256$ & 6.101 & 2.1 & 2.0 & 0.9 & 1.0 \\
\hline Crowd & $512 \times 512$ & 5.927 & 21.7 & 18.6 & 11.3 & 12.0 \\
\hline Crowd* & $256 \times 256$ & 5.362 & 0.7 & 0.8 & 0.9 & 0.8 \\
\hline
\end{tabular}

“*”Smaller images are obtained by downsampling the corresponding larger image

\subsection{Conclusion}

We have proposed PPAM - prediction by partial approximate matching for context modeling in lossless image compression. Rather than using exact contexts, PPAM models the contexts in an image in an approximate manner, based on $k$-approximate matching. Algorithms are proposed for efficient searching for approximate contexts using the PPAM contexts tree. To further reduce computational costs, the potentially huge contexts are clustered off-line, such that the required conditional probabilities can be determined at compression time using a simple look-up table. PPAM outperforms the original PPM method in contexts modeling for natural images. The PPAM lossless image compression algorithm produced competitive performance in context modeling when compared with other state-ofthe-art lossless image coding algorithms. The average coding performance is better than that of EDP and JPEG-LS on the same prediction error sequence. The performance of PPAM can be further improved by developing an improved context quantization algorithm and by using more sophisticated image clustering techniques. 


\section{Chapter 3: 2D Neurite Centerline Extraction}

\section{Related papers by Yong Zhang for this Chapter:}

(1) Y. Zhang, X. Zhou, A. Degterev, M. Lipinski, D. Adjeroh, J. Yuan, and S. T. C. Wong, "Automated neurite extraction using dynamic programming for highthroughput screening of neuron-based assays," NeuroImage, under revision, 2005.

(2) Y. Zhang, X. Zhou, A. Degterev, M. Lipinski, D. Adjeroh, J. Yuan, and S. T. C. Wong, "A novel tracing algorithm for high throughput imaging screening of neuron based assays," Journal of Neuroscience Methods, accepted for publication, 2006. 


\subsection{Introduction}

Knowledge and tools from cellular and molecular biology is playing an increasingly important role in assisting researchers in the pharmaceutical industry in studying the mechanisms of diseases and in finding better drug hits and leads. High-throughput screening (HTS) technology has recently emerged as an important drug discovery tool for identification of biological probes and drug leads by screening large volume, diverse biochemical and cellbased assays [34-41]. The power of the high-throughput screening comes from the automated fluorescence microscopy imaging techniques that make it possible and facile to visualize the complex biological processes at the cellular and molecular levels and allow speedy and economic acquisition of such high throughput neuron image data. This technology generates huge amounts of fluorescence microscopy images, with each image containing several million pixels. Accessing these data to extract and compose useful information requires efficient and cost-effective image analysis tools that involve minimum human interaction, the image processing task of which has been a challenging problem for years.

In the context of high-throughput screening of neuron-based assays, the fundamental goals of neuron image processing include: 1) measuring the length of the total neurite segments, and 2) detecting and segmenting neuron cells. In this paper, we consider the problem of automatically extracting and labeling all the neurite segments in a fluorescence microscopy neuron image. We observe that an individual neurite segment can be treated as a bright elongated wire-like structure surrounded by dark background; in this way, the problem is equivalent to the problem of extracting line structures from digital images.

The existing research work on extraction of line structures in digital images can be roughly categorized into three groups. The first group of algorithms is generally referred to as tracing-based algorithms [1, 42-47]. When extracting a single neurite segment, these 
algorithms start from an automatically selected seed point and detect a series of points along the centerline of the segment by estimating the local direction of each point. Each centerline point is traced based on its previous detected centerline point in a recursive manner until certain stopping conditions are satisfied. This group of algorithms is efficient since it only processes the pixels that are close to the line structures. Another advantage is that no further linking algorithm is needed since the points on the neurite segment can be linked and labeled during the tracing process. However, since most of these algorithms consider only the grayscale values and their estimation of the local directions is not robust, their performance on line extraction is quite low for images with poor quality or of complex line structures.

The second group of algorithms on line extraction is based on the concept of line-pixel detection [2, 48-56]. For each pixel, the algorithms consider local geometric properties of the lines, such as ridges and ravines, as well as their local orientations. The line pixels can be extracted accurately by modeling the 2-D line profiles and examining its first and second derivatives using the Gaussian smoothing kernel [57-59]. After detecting all the individual line pixels, additional effort is needed to link them together [2]. Although highly precise on extracting lines, these algorithms suffer a drawback that thin lines or lines with low intensity contrast may be smoothened out unintentionally by the Gaussian kernel, resulting in the loss of the corresponding neurite segments. Furthermore, they cannot resolve issues of discontinuities since there are no line pixels found in the breaking areas.

The third group is searching-based algorithms [3, 60-63]. Given each pair of starting point and ending point, these algorithms extract the line by searching for an optimal path from the starting point to the ending point. An optimal path can be found by calculating the globally minimal cumulative cost using a predefined cost function [3]. This is also referred to as live wire algorithm based on dynamic programming technology. Although robust against noise and discontinuities in line structures, these algorithms are unable to extract a line when 
a starting point is located within a large bright region with intensity values evenly distributed (usually a region of neuron cell body). They are computationally expensive since they search for and find an optimal path for every pixel in the image, not only for the ending point. The requirement of manually selecting starting points and ending points makes it certainly impractical for high throughput screening applications.

Based on the aforementioned observations and increasing development of highthroughput screening of neuron-based assays, we believe that it is necessary to develop an automated image analysis algorithm to extract and label the neurite segments accurately and completely with minimum user intervention. In this chapter, we propose a novel algorithm for automatic labeling and tracing of neurite segments in microscopy neuron images. The algorithm selects the starting and ending points automatically and links the line using dynamic programming techniques. Sections 2 and 3 describe the automatic selection of starting points and ending points while the linking algorithm using dynamic programming is presented in Section 4. Section 5 shows the experimental results and the validation method used to verify the extraction performance. The selection of the parameters and their effect on performance are also discussed. Section 6 presents our conclusions.

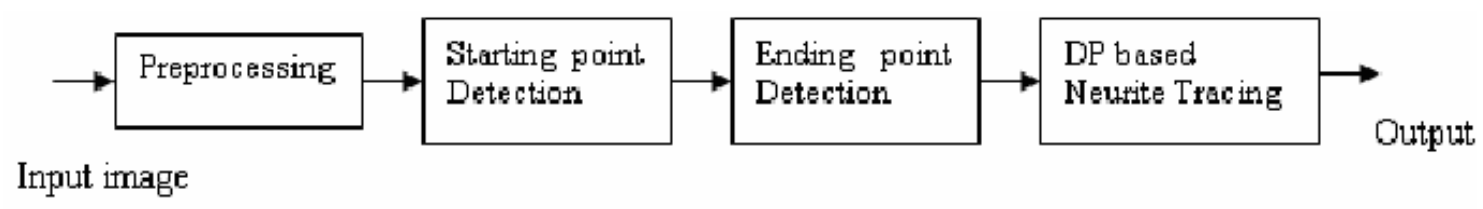

Figure 8. The block diagram for the neurite tracing algorithm using DP

\subsection{Starting point selection}

We present in Figure 8 a block diagram for our proposed algorithm. The input images are pre-processed to remove undesirable features and enhance branching structures. The starting points and the ending points of the neurite segments can be automatically detected so 
that human interaction is no longer required. The points are then linked automatically using dynamic programming technique. Each step is discussed in detail in the following subsections.

A starting point, also called a seed point, is a point on or near the centerline of a neurite segment. To extract all the neurite segments, at least one starting point should be selected on each individual segment. More than one starting point is needed in cases where the neurite structure is complex or the intensity contrast is very low along the neurite segment.

When selecting the starting points, only $N$ horizontal lines and $M$ vertical lines in the neuron image are considered. The pixels on each of the horizontal or vertical lines are first processed by a low-pass filter in the form of $\left[\begin{array}{lll}0.25 & 0.5 & 0.25\end{array}\right]^{T}$, an approximation of the 1-D Gaussian kernel. Since a discrete approximation in this form involves only local fixed point shifting and adding operations, the computational cost for the convolution can be significantly reduced. Thus, high-frequency components are removed from the sequence of pixels. After low-pass filtering, local intensity maxima are searched and detected using a window of size $W$ along each line. $W$ should be chosen so that at least one maximum is detected on each neurite segment. Thus, a small value is desired. In our work, we use the minimum expected neurite width as the value for $W$. Such a small window size may leads to multiple local maxima at locations where the neurite segments are very wide. This is how redundant seed points are detected. We believe that it is necessary to use redundant seed points so that the extraction is complete, partially because our targeted neuron images have very complicated neurite structures. The starting points will be selected among the local maxima detected on the set of grid lines.

We select the starting points based on the following two criteria:

Criterion 1: the local maxima $I_{m}$ has an intensity value that is larger than or equal to a threshold value $T_{I}: I_{m} \geq T_{I}$. 
The threshold value is determined by the intensity values of the image: $T_{I}=\mu_{I}+\sigma_{\mu}$, where $\mu_{I}$ is the median intensity value of all the pixels, and $\sigma_{\mu}$ is the standard deviation calculated based on $\mu_{I}$. This way, we pick up the pixels having intensity values that are above the average intensity of the image. However, those pixels whose intensity values are below the averaged intensity value and are on or near neurite segments will be ignored. Thus we need the second criterion for these pixels.

Criterion 2: the local maximum, $I_{m}$, has a local signal-to-noise ratio, $S N R_{m}$, that is larger than or equal to a threshold value $T_{S N R}: S N R_{m} \geq T_{S N R}$.

$S N R_{m}$ is calculated based on the point $m$ and its local neighborhoods $N_{m}$, a $10 \times 10$ block of pixels centered at the point $m$. We calculate a local threshold value $T_{N}$ for the neighborhoods $N_{m}$ using Otsu's algorithm [64]. The pixels in $N_{m}$ are grouped as the foreground if their intensity values are larger than or equal to $T_{N}$. The rest of the pixels in $N_{m}$ are then grouped as the background. By defining the mean value of the intensities of the foreground pixels and background pixels as $\mu_{f}$ and $\mu_{b}$ respectively, and the standard deviation of the background pixels as $\sigma_{b}$, the local signal-to-noise ratio $S N R_{m}$ is calculated as follows:

$$
S N R_{m}=\frac{\mu_{f}-\mu_{b}}{\sigma_{b}}
$$

the threshold value $T_{S N R}$ is determined by the mean value of all the local signal-to-noise ratio calculated by (1) for all the local maximum point $m$ detected previously:

$$
T_{S N R} \geq \underset{m}{\operatorname{median}}\left\{S N R_{m}\right\}
$$


In other words, if a local maximum point has an intensity value that is below the threshold, we still keep it as a starting point if its local SNR is above the average SNRs among all the local maxima points.

\subsection{Ending point selection}

An ending point is identified by iteratively tracing a single neurite segment from the selected starting point until the end of the segment. The track does not need to be accurate or smooth; since the accuracy and smoothness of the track will be achieved by the linking process based on dynamic programming technique. Thus we need to design a rapid but rough tracing method which has robust stopping criteria for selection of the ending points. A tracing from the current point $p_{i}$ to the next point $p_{i+1}$ can be described as estimating the local direction $d_{i}$ for $p_{i}$, and tracing along $d_{i}$ to find the point $p_{i+1}$. The tracing starts at one of the starting points, steps forward from one point to another along the neurite segment, and stops when the stopping conditions are satisfied, at which the ending point is identified. The question is then how to estimate the local direction of a pixel in the image.

The local direction $d_{i}$ of point $p_{i}$ is estimated by correlating the neighborhoods of $p_{i}$ with a set of 2-D directional correlation patterns. Early usage of such directional correlation patterns can be found in [65] and later they were widely used in [1, 43]. A correlation pattern,

also called a template, is defined as a 2-D filter that calculates a low-pass differential perpendicular to the direction of a line and low-pass averaging along the direction of the line. An example of such patterns, designed to calculate the correlation along the $\mathrm{x}$-axis direction

( $0^{\circ}$ direction), is shown in Figure 9. Obviously a point on a line structure along the $\mathrm{x}$-axis direction will have the maximum correlation with such a pattern among all the 2-D directions that a line structure could have. The patterns at other directions can be generated by rotating 
the one at $0^{\circ}$ direction. Notice that the first two lines of the pattern in the figure have

\begin{tabular}{|l|l|l|l|l|l|}
\hline 1 & 1 & 1 & 1 & 1 & 1 \\
\hline 2 & 2 & 2 & 2 & 2 & 2 \\
\hline 0 & 0 & 0 & 0 & 0 & 0 \\
\hline-2 & -2 & -2 & -2 & -2 & -2 \\
\hline-1 & -1 & -1 & -1 & -1 & -1 \\
\hline
\end{tabular}

Figure 9. The correlation kernel at zero-degree direction

opposite signs compared to the last two lines of the pattern. This means that the maximum correlation occurs at the boundary of the line structure. Thus the local direction of a centerline point can be estimated by correlating the corresponding points on or near the edge of the line structure, which is achieved by searching for the edge points along the two directions perpendicular to the local direction.

Since the local direction is unknown, we need to do searching for all the possible 2-D directions. To simplify the process, all the 2-D directions are quantized into 16 directions [1], each represented by an integer number. We denote the quantized direction as $d^{k}$ where $k=0,1, \ldots 15$. There exists a pair of patterns for each $d^{k}$, one for the left edge and the other for the right edge. They are denoted as $T_{L}^{k}, T_{R}^{k}$, with subscripts $L, R$ indicating the direction toward the left edge or the right edge. The two directions perpendicular to $d^{k}$ are denoted as $d_{L}^{k}$ and $d_{R}^{k}$. The correlation $g$ between the patterns $T_{L}^{k}, T_{R}^{k}$ and a point $p_{i}$ with the coordinate $(m, n)$ is calculated as follows:

$$
\begin{aligned}
& g_{L}^{k}(m, n)=\sum_{x, y} N_{p}(m+x, n+y) * T_{L}^{k}(x, y) \\
& g_{R}^{k}(m, n)=\sum_{x, y} N_{p}(m+x, n+y) * T_{R}^{k}(x, y)
\end{aligned}
$$


where $N_{p}$ is the neighborhoods of $p_{i}$ selected as a $5 \times 6$ block of pixels with $p$ centered at the leftmost column. “*” denotes the multiplication operator. Such a correlation operation obviously indicates the "matchness" between the pattern and the image at each point. For each left direction $d_{L}^{k}$ and its pattern $T_{L}^{k}$, we search for a maximum correlation calculated by (3) beginning from $p_{i}$ until a pre-defined maximum possible width $W_{\max }$, resulting in a total of 16 such maximum correlations. The highest correlation represents the best match and thus the corresponding direction can be selected as the estimation of the local direction. The same search is also applied along the right direction $d_{R}^{k}$. The highest correlations are obtained as follows:

$$
\begin{aligned}
& G_{L}=\max _{k,(m, n)}\left\{g_{L}^{k}(m, n)\right\} \\
& G_{R}=\max _{k,(m, n)}\left\{g_{R}^{k}(m, n)\right\}
\end{aligned}
$$

where $G_{L}, G_{R}$ denotes the highest correlations along the left and right directions respectively. The points at which the highest correlations occur are identified as the edge points and are denoted as $p_{L}, p_{R}$ :

$$
\begin{aligned}
& p_{L}=\left(m_{L}, n_{L}\right)=\underset{k,(m, n)}{\arg \max }\left\{g_{L}^{k}(m, n)\right\} \\
& p_{R}=\left(m_{R}, n_{R}\right)=\underset{k,(m, n)}{\arg \max }\left\{g_{R}^{k}(m, n)\right\}
\end{aligned}
$$

since the current point $p_{i}$ may not be on or near the centerline, its location needs to be adjusted. The new location of $p_{i}$ resides at the middle point of the line between $p_{L}$ and $p_{R}$. The local direction for $p_{i}$ is estimated as the direction whose correlation is the highest in a total of 32 maximum correlations:

$$
d_{i}=\underset{k}{\arg \max }\left\{G_{L}, G_{R}\right\}
$$

The next point $p_{i+1}$ is traced from $p_{i}$ along the direction $d_{i}$ as follows: 


$$
p_{i+1}=p_{i}+d_{i}
$$

Once the local direction of a point is determined, the local direction for the next point can be estimated from the three directions that are similar to the previous one. This is based on the assumption that the directions change smoothly along the orientation of the neurite. If the estimated local direction is $d_{i}=d^{k}$ for point $p_{i}$, then the local direction for $p_{i+1}$ will be estimated from $\left\{d^{k-1}, d^{k}, d^{k+1}\right\}$. In this way the running time is further reduced.

Since a starting point may be located at the middle of a line structure, at least two ending points need to be identified. This is achieved by estimating two local directions for the starting point. The second local direction is selected as the one corresponding to the second highest correlation in the 32 maximum correlations calculated by (3). The angle between the two local directions is $180^{\circ} \pm 22.5^{\circ}$ if quantization errors are considered.

Tracing stops when it reaches the boundary of the image or a cell body, or it goes from the foreground into the background. The following criteria capture these situations:

Criterion 1: The next centerline point has been traced before, or it is a starting point, or its neighboring point has been traced before.

Criterion 2: The next centerline point lies on or outside the boundary of the image, or it lies on or inside a soma region (the method to detect soma regions is discussed in [66]).

Criterion 3: The number of tracing steps for a single neurite reaches a pre-defined maximum value, or it is below a pre-defined minimum value.

Criterion 4: The next centerline point has the highest maximum correlation that is below a threshold value $T$.

To determine the threshold value for Criterion 4, we consider a method proposed in [1]. This method is based on the dynamic range of the intensity values on the grid line where the starting point is selected. Since the intensity values usually change along the line being traced, the threshold value by that method terminates the tracing too early. For example, if the 
intensities decrease along the line, a threshold value determined at the starting point is obviously too high to completely trace the entire neurite segment. In this paper, we propose a revised method that can handle this situation. At each traced centerline point $p_{i}$, a threshold value $T_{p}$ is calculated based on the method by [1]. We compare $T_{p}$ with the smallest threshold value calculated at all the starting points in the image, and pick the maximum one as the threshold value $T$ that will determine if the tracing should be terminated at point $p_{i}$.

That is,

$$
T=\max \left\{\min _{s}\left\{T_{s}\right\}, T_{p}\right\}
$$

where $S$ is the set of all the starting points. The calculation of a threshold value on the right hand side of (8) is applied on a single line. The line for calculating $T_{p}$ is determined by the local direction $d_{i}$ of $p_{i}$. If $d_{i}$ points vertically or horizontally, the line is the vertical line or the horizontal line with $p_{i}$ on it. Otherwise both vertical and horizontal lines are used to calculate $T_{p}$. The line for calculating $T_{s}$ is simply the one that is used to select the starting point. Since the minimum value among all the threshold values is used, the early termination of a tracing is avoided. The larger one between $T_{s}$ and $T_{p}$ is used to ensure that the tracing stops when it enters the background. Using only $T_{p}$ will lead to an infinite loop or the tracing is terminated too late.

\subsection{Center-line extraction using dynamic programming}

\subsubsection{Introduction to dynamic programming}

Dynamic programming [67] is an optimization procedure that is designed to efficiently search for the global optimum of an energy function; it is an algorithmic technique based on a 
recurrent formula and some starting states. The problem can usually be divided into stages with a decision required at each stage. Each stage has a number of states associated with it. The decision at one stage transforms one state into a state in the next stage. Given the current state, the optimal decision for each of the remaining states does not depend on the previous states or decisions. However, the sub-solution of the problem at each stage is constructed from the previous ones, which is distinguished from the divide and conquer algorithm. There exists a recursive relationship that identifies the optimal decision for stage $i$, given that stage $i+1$ has already been solved, and the final stage must be solvable by itself. The dynamic programming solutions have a polynomial complexity which assures a much faster running time than other techniques, such as the backtracking and brute-force. The most important and difficult issue in dynamic programming is to take a problem and determine stages and states so that all of the above characteristics hold. More details and examples can be found in [68]

In the field of computer vision, dynamic programming has been widely used to optimize the continuous problem and to find stable and convergent solution for the variational problems $[69,70]$. Many boundary detection algorithms use dynamic programming as the shortest or minimum cost path graph searching method [71, 72]. The problem of such a technique, if applied on image segmentation, is that multiple user inputs are required in case of parameter adjustment, change of initial conditions, or any other situations that require the restart of the application. The live wire technique, an interactive optimal path selection algorithm, was developed to solve the problem which can find multiple optimal paths from one seed point to every other pixel in the image, and display the minimum cost path from the current point to the seed point on-the-fly [60-63]. Dynamic programming, however, is more suitable for the neurite extraction problem, since the starting point and the ending point are known. The linking between a pair of points can be considered as a problem of searching for 
an optimal path between the two points, a problem that can be solved using dynamic programming approach, if the energy function is defined properly.

\subsubsection{Optimal path selection}

We denote the local energy cost as $c_{L}(p)$ (which is related to the line structure and will be discussed later in this section) for point $p$ and the direction energy cost as $c_{D}(p, q)$ representing the linking cost from point $p$ to one of its 8-neighbors $q$. Then the total cost $C$ from $p$ to $q$ is given by:

$$
C(p, q)=c_{L}(p)+c_{L}(q)+c_{D}(p, q)
$$

if $P\left(p_{0}, p_{K}\right)$ denotes a path from a starting point $p_{0}$ to an ending point $p_{K}$, the total cost for such a path will be:

$$
C\left(p_{0}, p_{K}\right)=\sum_{i=0}^{K} c_{L}\left(p_{i}\right)+\sum_{j=0}^{K-1} c_{D}\left(p_{j}, p_{j+1}\right)
$$

There exists $n_{i}$ number of candidate points for each point $p_{i}(i=1, \ldots K-1)$ between $p_{0}$ and $p_{K}$. That is, $p_{i} \in\left\{p_{i}^{1}, p_{i}^{2}, \ldots p_{i}^{n_{i}}\right\}$. The problem of finding an optimal path from $p_{0}$ to $p_{K}$ is equivalent to searching for a path $\hat{P}\left(p_{0}, p_{K}\right)=\left\{p_{0}, p_{1}, \ldots, p_{K-1}, p_{K}\right\}$ that minimizes $C\left(p_{0}, p_{K}\right):$

$$
p_{1}, \ldots, p_{K-1}=\underset{P}{\arg \min }\left\{C\left(p_{0}, p_{K}\right)\right\}
$$

by rewriting equation (10) as a multistage process:

$$
\begin{aligned}
& C\left(p_{0}, p_{0}\right)=c_{L}\left(p_{0}\right) \\
& C\left(p_{0}, p_{i}\right)=C\left(p_{0}, p_{i-1}\right)+c_{L}\left(p_{i}\right)+c_{D}\left(p_{i-1}, p_{i}\right)
\end{aligned}
$$


where $i=1, \ldots, K$. Thus, with the dynamic programming approach, the problem of finding $\hat{P}\left(p_{0}, p_{K}\right)$, the optimal path can be divided into stages and each pool of points on the path between $p_{0}$ and $p_{K}$ is regarded as a stage. All the possible candidate points for each stage become the possible states of that stage. Thus a path can be constructed as illustrated in Figure 10, with each circle representing a point. Starting from point $p_{0}$, we calculate for each state its minimum cost accumulated from the previous stage as follows:

$$
\begin{aligned}
& C_{m}\left(p_{0}\right)=0 \\
& C_{m}\left(p_{i}^{j}\right)=\min _{t=1, \ldots n_{i-1}}\left\{C_{m}\left(p_{i-1}^{t}\right)+C_{L}\left(p_{i}^{j}\right)+C_{D}\left(p_{i-1}^{t}, p_{i}^{j}\right)\right\}
\end{aligned}
$$

where $p_{i}^{j}$ is the $j$-th candidate point (state) for the $i$-th stage, and $C_{m}\left(p_{i}^{j}\right)$ is the minimum cost if linking $p_{0}$ and $p_{i}^{j}$. The candidate point which produce $C_{m}\left(p_{i}^{j}\right)$ is then stored for future retrieval of the optimal path. The cost of the starting point is set to zero, even though it may have a non-zero local cost. The optimal path can be obtained by tracing from the ending point back to the starting point:

$$
p_{i-1}=\underset{t}{\arg \min }\left\{C_{m}\left(p_{i-1}^{t}\right)+c_{L}\left(p_{i}\right)+c_{D}\left(p_{i-1}^{t}, p_{i}\right)\right\}
$$

where $i=K, \ldots 2$ and $t=1, \ldots n_{i-1}$.

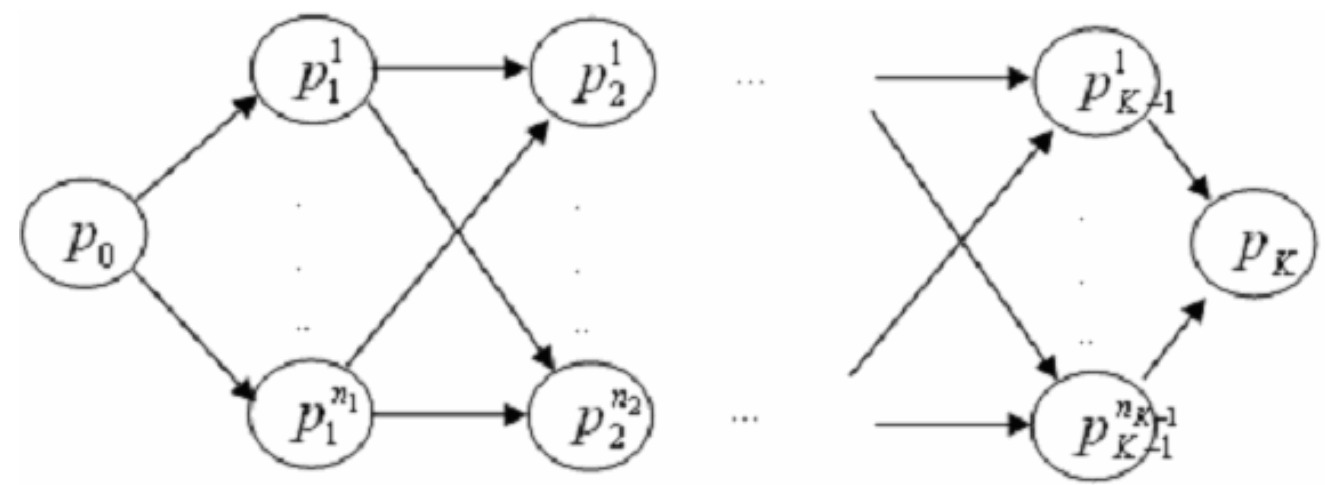

Figure 10. A example of finding the optimal path using the DP 
The selection of the candidate points for each stage, however, is a difficult problem for the above dynamic programming approach, since in 2-D images one point may have many linking relationships to another point. For example, one point can directly connect to another point via the diagonal direction, or it may connect to the third point vertically and then connect the targeted point horizontally, as shown in Figure 11. Here we use the idea of Dijkstra's unrestricted graph search algorithm [73] to define the linking relationships among the candidate points. With this method a list $L$ of active points is maintained by adding unprocessed points to the list in an increasing order of the cumulative cost. The calculation of the cumulative cost is applied on the point $p$ with the minimum cost in $L$. Basically the 8 neighbors of $p$ are considered the candidates of the next stage if they have not been earlier processed. If a neighboring point $N_{p}$ is in $L\left(N_{p}\right.$ is a state in a previous stage) and has a cumulative cost that is larger than that if linking directly $p$ and $N_{p}$, then $N_{p}$ is removed from $L$, which means the previous calculation for $N_{p}$ is invalid, and $N_{p}$ can be reconsidered as a candidate for the new stage. Thus a new connecting relationship is created between the seed point and $N_{p}$ by linking directly $N_{p}$ and $p$. By adjusting the candidate points in each stage based on the cumulative cost, each pixel in the 2-D image will have an optimal path to the starting point, including the ending point. The optimal path can then be retrieved using (14) by back tracing from the ending point.

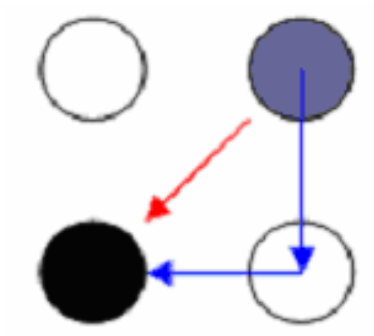

Figure 11. Linking relationships between two points. 


\subsubsection{Complexity Analysis}

By recording the interim results, the dynamic programming technique has a running time of $\mathrm{O}\left(\mathrm{N}^{2}\right)$ for solving combinatorial problems compared to a traditional recursive algorithm that has a $\mathrm{O}\left(2^{\mathrm{N}}\right)$ running time, where $\mathrm{N}$ is the size of the problem. However, the running time of dynamic programming may dramatically increase when applied in highthroughput screening, due to the huge amount of neurite segments presented in a neuron image. The experiments show that a typical $1000 \times 1000$ neuron image may have up to 500 1000 neurite segments, so it is reasonable to assume that the total number of neurites in an image is proportional to the image size $\mathrm{N}_{x}\left(\mathrm{~N}_{x}=N \times N\right.$ for an $N \times N$ image). The dynamic programming then needs $\mathrm{O}\left(\mathrm{N}_{x}^{2}\right)$ for each starting point on an individual neurite segment since it calculates an optimal path between the starting point and all the other pixels in the image, resulting in a total of $\mathrm{O}\left(\mathrm{N}_{x}^{3}\right)$ running time if a single neuron image is processed. Obviously this is not feasible for high-throughput application. To address this problem, we apply dynamic programming only on a small area of the image which is defined by the starting point, the ending point, and the edge points detected during the ending point tracing process. The searching area (defined as a top-left pixel $I_{T L}$ and a bottom-right pixel $I_{B R}$ in the image) is selected as follows:

$$
\begin{aligned}
& I_{T L}=\left(x_{T L}, y_{T L}\right)=\left(\min \left\{x_{C}, x_{E}\right\}, \min \left\{y_{C}, y_{E}\right\}\right) \\
& I_{B R}=\left(x_{B R}, y_{B R}\right)=\left(\max \left\{x_{C}, x_{E}\right\}, \max \left\{y_{C}, y_{E}\right\}\right)
\end{aligned}
$$

where $\left(x_{C}, y_{C}\right)$ is the coordinate of all the center-line points detected and $\left(x_{E}, y_{E}\right)$ is the coordinate of all the edge points (left and right edge points) detected during the tracing process. Since a neurite segment should not cross itself, its maximum length $L_{\max }$ is: 


$$
\begin{aligned}
L_{\max } & =(N+N)+(N-1+N-1)+\ldots+(2+2)+(1+1) \\
& =2\left[\frac{N(N-1)}{2}\right]=N(N-1) \\
& =O\left(\mathrm{~N}_{x}\right)
\end{aligned}
$$

At each point we search within a neighborhood of size $m(m=8$ or $m=4)$, so the cost for linking one neurite segment is $\mathrm{O}\left(\mathrm{mN}_{x}\right)$. If the number of neurite segments is estimated as

$\sqrt{\mathrm{N}_{x}}$, the total running time is $\mathrm{O}\left(m \mathrm{~N}_{x} \sqrt{\mathrm{N}_{x}}\right)$. Since $m$ can be fixed, we have the total cost $\mathrm{O}\left(\mathrm{N}_{x} \sqrt{\mathrm{N}_{x}}\right)$

\subsubsection{Designing the energy function}

In designing the energy function for the optimal path searching, we have adopted the method proposed in [3]. The second-order directional derivative has been widely used for detecting ridges and ravines in digital images. With this method, the image is treated as a 2-D noisy function and its first- and second-order derivatives are estimated as the image convolved with the derivatives of the 2-D Gaussian kernel. The Gaussian kernel and its derivatives in 1-D case with the standard deviation $\sigma$ are given by:

$$
\begin{aligned}
& G(x, \sigma)=\frac{1}{\sigma \sqrt{2 \pi}} \exp \left(-\frac{x^{2}}{2 \sigma^{2}}\right) \\
& G^{\prime}(x, \sigma)=\frac{-x}{\sigma^{3} \sqrt{2 \pi}} \exp \left(-\frac{x^{2}}{2 \sigma^{2}}\right) \\
& G^{\prime \prime}(x, \sigma)=\frac{x^{2}-\sigma^{2}}{\sigma^{5} \sqrt{2 \pi}} \exp \left(-\frac{x^{2}}{2 \sigma^{2}}\right)
\end{aligned}
$$

Thus the derivatives of an image, if treated as a 1-D function $I(x)$, can be estimated as follows:

$$
I^{\prime}(x)=I(x) * G^{\prime}(x, \sigma), \quad I^{\prime \prime}(x)=I(x) * G^{\prime \prime}(x, \sigma)
$$


where "*" is the convolution operator. Considering a modified Hessian matrix whose form is given by:

$$
\left(\begin{array}{cc}
I_{x x}-\frac{1}{3} I_{y y} & \frac{4}{3} I_{x y} \\
\frac{4}{3} I_{x y} & I_{y y}-\frac{1}{3} I_{x x}
\end{array}\right)
$$

where $I_{x x}, I_{y y}, I_{x y}$ are the partial derivatives along the $x, y$ directions for a 2-D image $I$, the local directions of a ridge point are given by the eigenvectors of this matrix. Let $\lambda_{a}, \lambda_{b}$ denote the two eigenvalues and, $v_{a}, v_{b}$ the corresponding eigenvectors which satisfy $\left|\lambda_{a}\right|>\left|\lambda_{b}\right|$, it is known that the longitudinal direction of the ridge structure is the pointing direction of $v_{b}$ and its perpendicular direction is given by the direction of $v_{a}$. The local cost $c_{L}(p)$ for each ridge point $p$ is:

$$
c_{L}(p)= \begin{cases}1-\frac{\lambda_{a}}{\lambda_{a \min }}, & \text { if } \lambda_{a}<0 \\ 1 & \text { otherwise }\end{cases}
$$

where $\lambda_{a \min }$ is the minimum $\lambda_{a}$ over all the pixels in the image. Obviously the local cost reflects how close the ridge point is to the center of the line structure. That is, the closer the point is to the center, the lower the local cost will be. The cost $c_{D}\left(p_{1}, p_{2}\right)$ of linking two points $p_{1}, p_{2}$ is given by:

$$
c_{D}\left(p_{1}, p_{2}\right)=\frac{1}{2}\left(\sqrt{1-e_{b 1} e_{p_{2}-p_{1}}}+\sqrt{1-e_{b 1} e_{p_{1}-p_{2}}}\right)
$$

where $e_{b 1}$ and $e_{p_{2}-p_{1}}$ are the unit vector of $v_{b 1}$ and $p_{2}-p_{1}$. From formula (46) we see that the linking cost will be high in cases when sharp changes occur along the boundary direction and low in cases when the two linking points have similar gradient directions. The total cost $C\left(p_{1}, p_{2}\right)$ from $p_{1}$ to $p_{2}$ is defined as: 


$$
C\left(p_{1}, p_{2}\right)=\varepsilon c_{L}\left(p_{2}\right)+(1-\varepsilon) c_{D}\left(p_{1}, p_{2}\right)
$$

where $\varepsilon$ is a weighted parameter that has a value between 0 and 1 . Later in the result section we will discuss how this parameter affects the extraction performance. Since the ending points are already known, the problem of ignoring some neurite segments by the Gaussian smoothing kernel will be avoided.

\subsection{Results, validation, and discussion}

\subsubsection{Image acquisition}

The test images are provided by Yuan's Lab, one of our collaborators at the Institute of Chemistry and Cell Biology (ICCB), Harvard Medical School. ICCB has an excellent

high-throughput small molecule screening facility, one of the first high throughput screening facilities in academic centers. Many researchers have devoted their time and efforts to identifying potential compounds that could prevent damages and losses of synapses and neurites, known to be the early events in Alzheimer's disease (AD). One predominant view of the primary cause of $\mathrm{AD}$ is the abnormal accumulation of the $\mathrm{A}$ peptides, the primary component of the amyloid plaques. In order to study the role of A ...an experiment at Yuan's Lab was established using a sensitive and reproducible assay for amyloid neurotoxicity in mouse primary cortical neurons using either aggregated or soluble A . 1-40. Light microscopy was used to observe neuronal morphology following A treatment as compared to untreated controls. Recently they have further extended the analyses of the neurite loss following A . treatment to the use of high-throughput screening methodology developed at ICCB. They utilized cortical neurons stained with neuronal-specific antibody MAP2 and normalized to neuronal cell number by counterstaining with nuclear stain DAPI. Primary cortical neurons were left untreated or treated with $10 \quad$ M A $\quad 1-40$ in 384 well plates for 48 
hours, followed by staining with TUJ1 and Sytox Green. Cell images were acquired using Autoscope platereader at 20X resolution.

\subsubsection{Neurite extraction results}

Figure 12 and Figure 13 show two results for neurite segment extraction of two neuron images. Each test image has complicated neurite structures and manually labeling those neurite segments would require heavy laboring and huge time. Manual labeling may also ignore those neurite segments with very low intensity contrast presented in the test images. Our proposed algorithm can extract and label most neurite segments in the image and the quantification of those neurite can also be done automatically. The running time for extracting the neurite structures in an image with size $1360 \times 1200$ is 24.1 seconds with Pentium IV, 2.4GHz and $1024 \mathrm{MB}$ memory, compared to 24 minutes of running time if a typical live-wire based software is used to extract the same number of neurite segments from the same neuron image using the same computer.

We also compare the extraction performance between our proposed method and the NeuronJ, a method proposed by Meijering et al [3] and a plug-in tool of the ImageJ [74]. Figure 14 shows a partial image of neurite segments and two extraction results. Figure 14 (b) is the extraction result by NeuronJ. Note that the starting point and the ending point have to be specified by the user. Figure 14 (c) is the extraction result by our proposed method. The starting point and the ending points are automatically determined by the algorithm. It is clear to see that our proposed method has the extraction performance as accurate as that of NeuronJ and outperforms NeuronJ in terms of automation. 


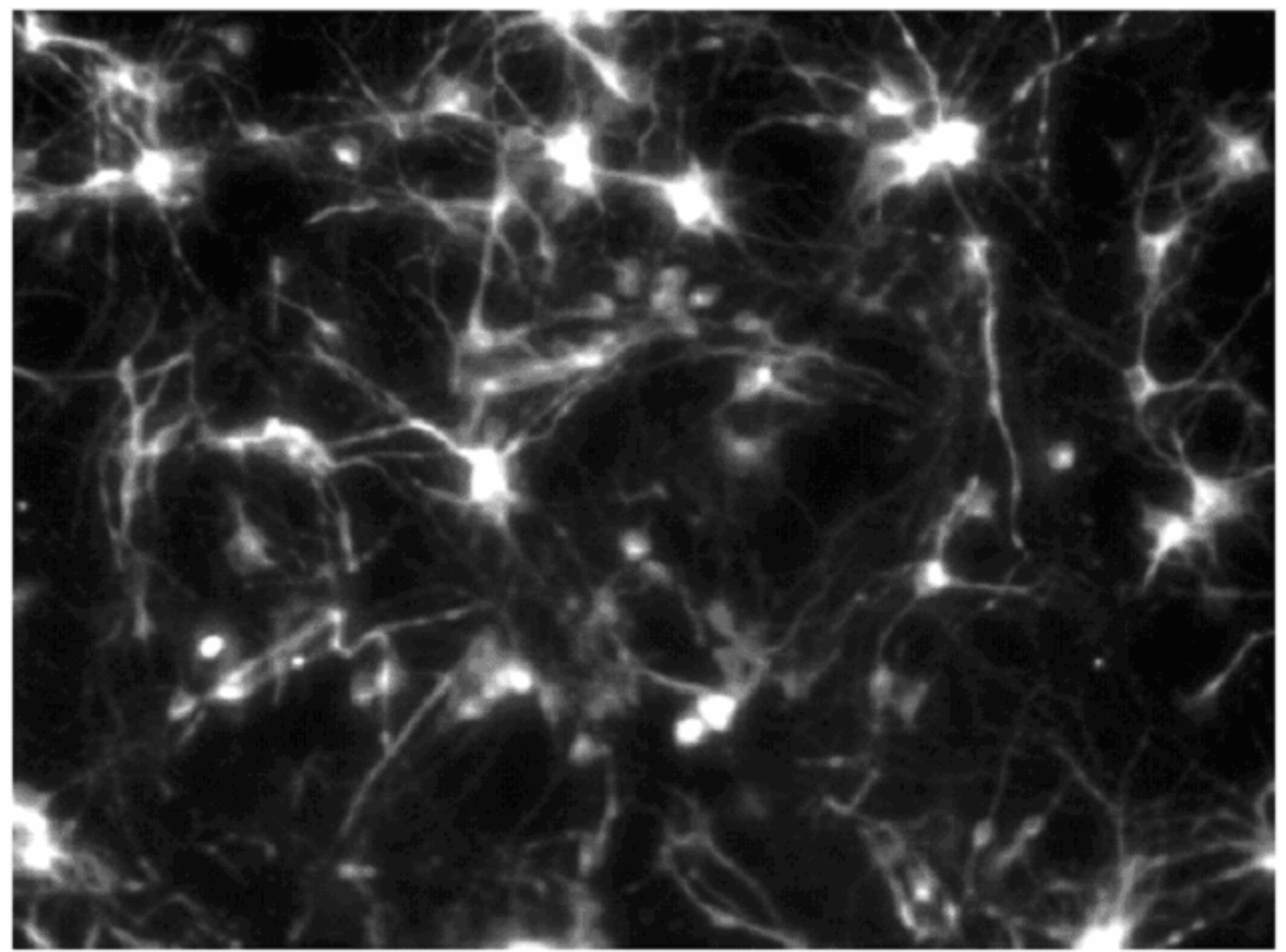

(a)

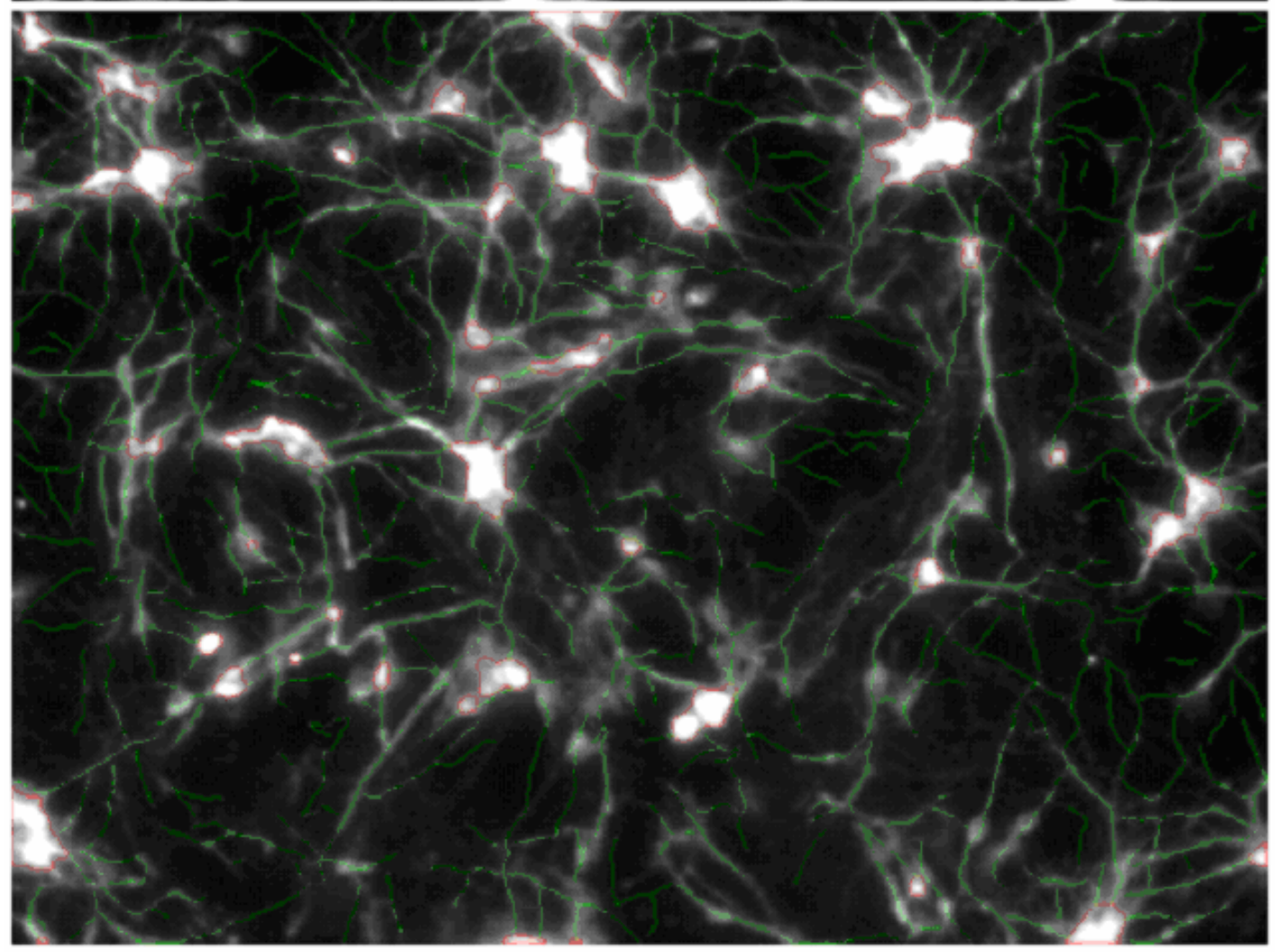

(b)

Figure 12. Sample results for neurite extraction. (a) original image; (b) result image with neurites labeled by green color and soma regions labeled by red color. 


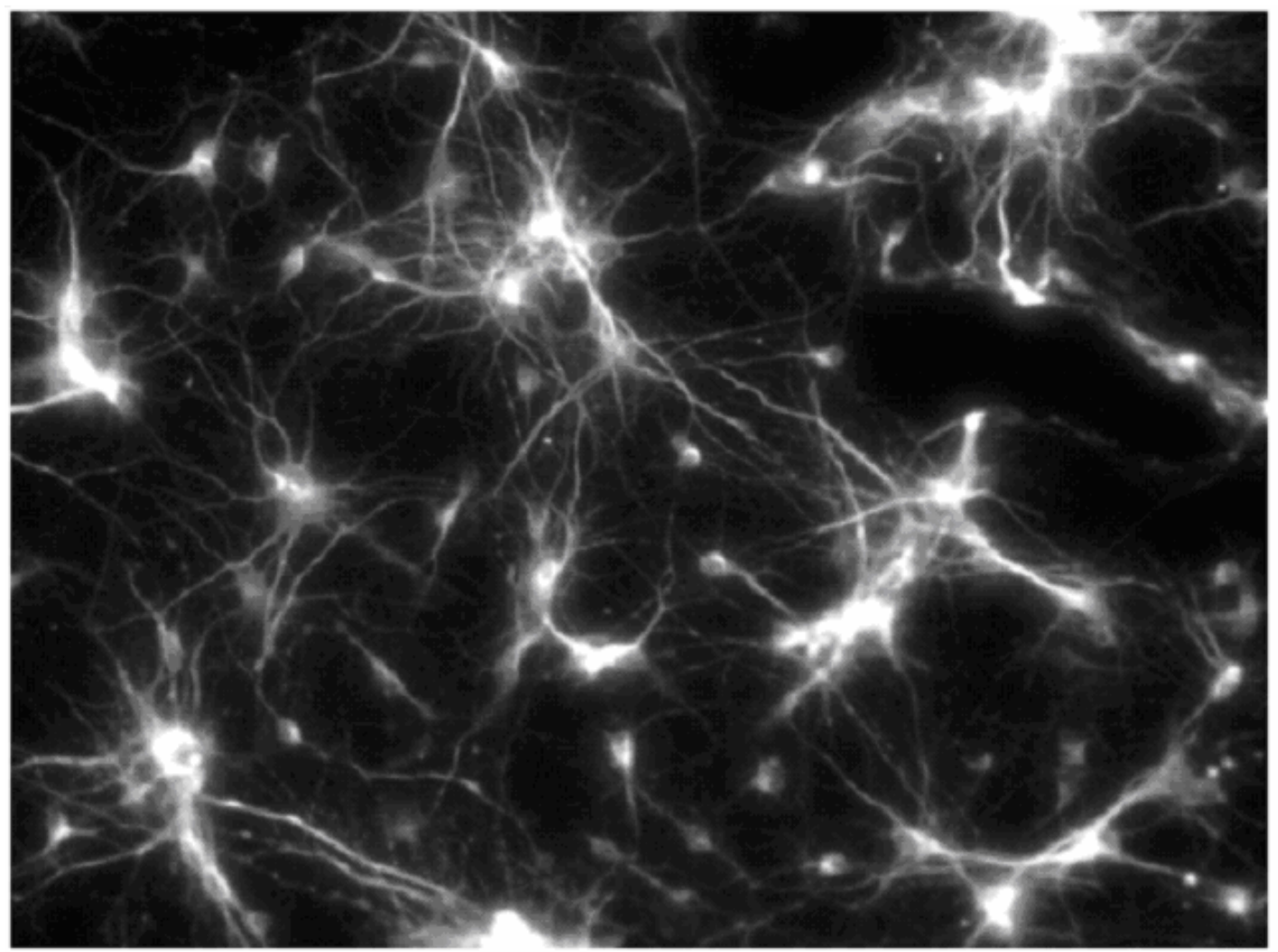

(a)

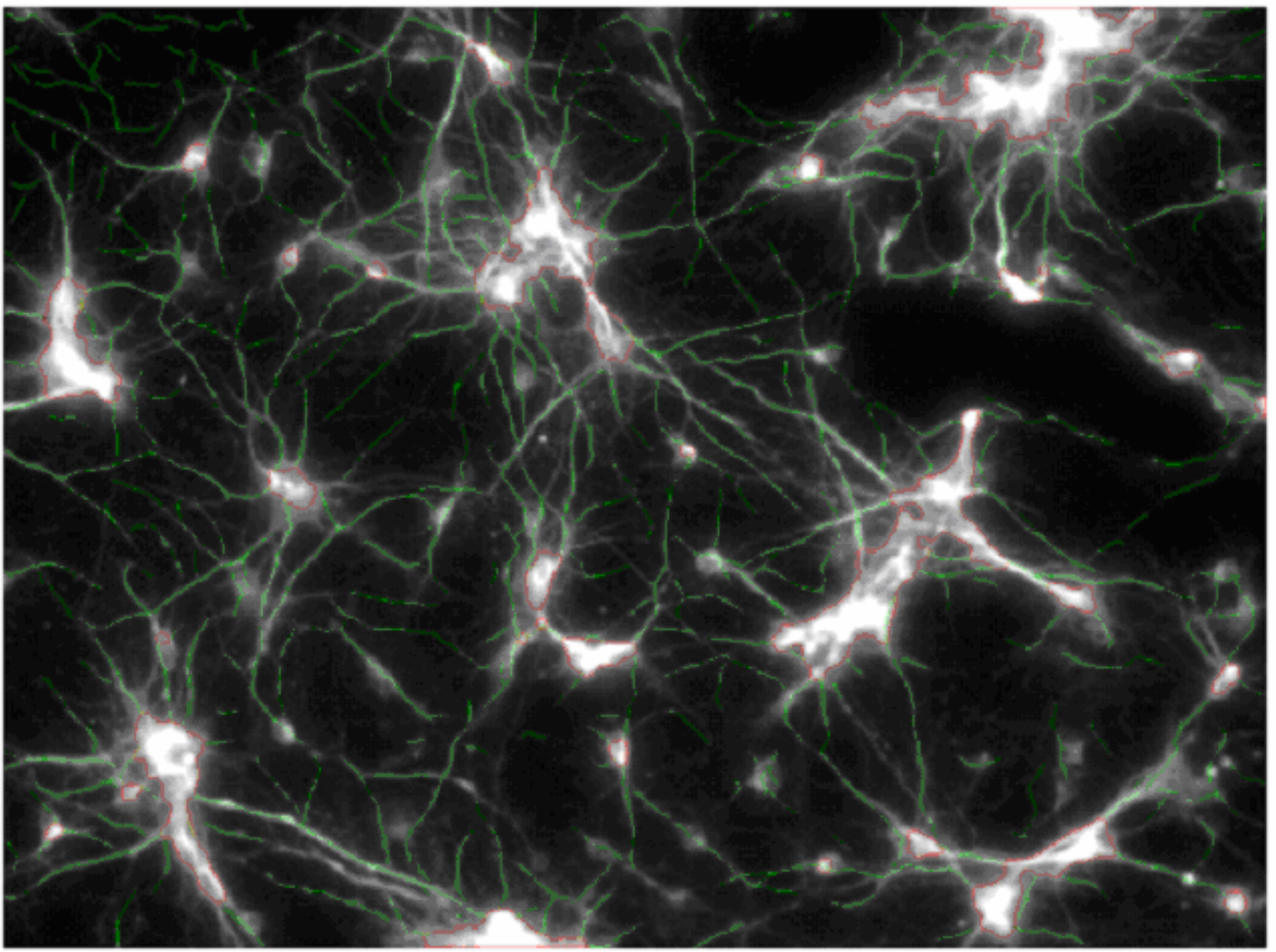

(b)

Figure 13. Sample results for neurite extraction. (a) original image; (b) result image with neurites labeled by green color and soma regions labeled by red color. 


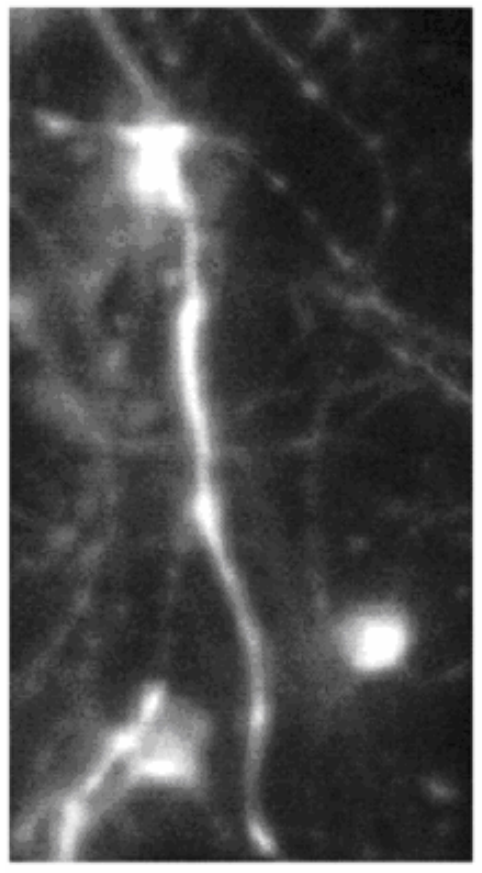

(a)

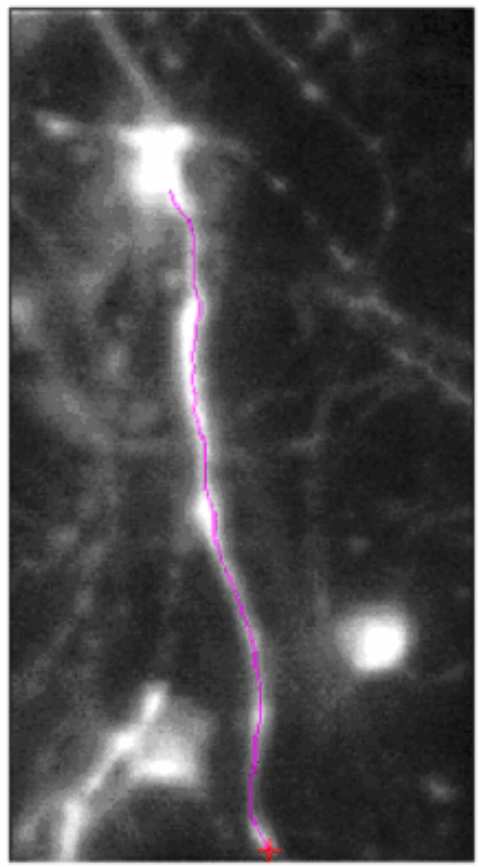

(b)

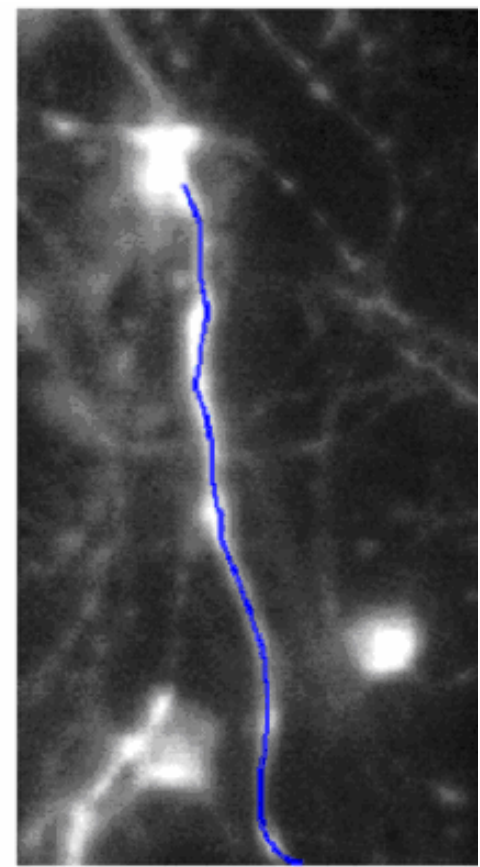

(c)

Figure 14. A partial image of neurite segment and two extracting results. (a) original partial image; (b) extracting result using NeuronJ; (c) extracting result using our proposed method.

\subsubsection{Validation}

It is a difficult problem to evaluate the algorithm for automated neurite tracing, because manual labeling always has bias and different people may have quite different results. If the manual tracing is performed in a completely independent way and blinded to the algorithm, then the quantitative difference between the manual results and the automated results will be always quite huge. For example, users may randomly pick up a starting point and draw a centerline from that point and stop at any location they want. In images with complicated branching structures, human users may select a branch that is different from the one selected by the algorithm, even though the overall labels for the two methods could be very similar if examined visually.

If the manual tracing is guided by the automated results, then it is guaranteed that both human users and the algorithm use the same starting and ending points and select the same 
routine when tracing the complicated branching structures. Based on that, the human users still judge independently where the centerlines are located. We believe that using this method, the algorithm can be evaluated effectively and efficiently from both visual inspection and quantitative comparison.

The following experiment is used to quantitatively evaluate the neurite extraction performance of our proposed algorithm. We apply our algorithm on 100 neuron images to extract neurite segments. Then a total of 20 soma regions and their neurite segments are randomly selected from those images. Two independent individuals manually labelled the neurite segments in the twenty soma regions. The manual labeling process is guided by the computer generated results so that the two results can be compared for validation. Length difference and centerline deviation are the quantities used for evaluating the difference between the manual results and the computer generated results. The length difference $(\phi)$ is defined as follows: $\phi=\left|1-\frac{L_{m}}{L_{c}}\right|$, where $L_{m}$ is the length of the neurite segment extracted manually, $L_{c}$ is the length of the neurite segment generated by the algorithm. The centerline deviation $(\varphi)$ is calculated as follows: $\varphi=\frac{\operatorname{Area}\left(l_{c}, l_{m}\right)}{L_{c}}$,

where $l_{c}$ and $l_{m}$ are the centerlines of the neurite segments extracted automatically and manually, respectively. Area $\left(l_{c}, l_{m}\right)$ represents the area of the region in pixels surrounded by $l_{c}$ and $l_{m}$. Given $\phi$ and $\varphi$, we use the following methods to validate our results:

- compare the mean and standard deviation of $\phi$ and $\varphi$ between the two observers;

- calculate the $p$-values of $\phi$ and $\varphi$ using the two-sided paired $t$-test;

- calculate the Pearson linear correlation coefficients of $\phi$ and $\varphi$; 
- calculate the cumulative distribution of $\phi$ and $\varphi$ using Kolmogorov-Smirnov goodness-of-fit hypothesis test [75] and compare the quantile-quantile plots of the two observers.

Table 13 lists the mean and standard deviation of $\phi$ and $\varphi$ for the two observers, as well as the p-values between them. The results show that the manual extractions between the two observers are very similar. Table 14 shows the Pearson linear correlation coefficients of $\phi$ and $\varphi$ among the algorithm and the two observers. The coefficients indicate that there exist strong correlations between the computer generated results and Observer 1's results, the computer generated results and Observer 2's results, Observer 1's results and Observer 2's results. Figure 15 shows the box plots of the mean and standard deviation of $\phi$ and $\varphi$ for the two observers, indicating that the quantitative results from the two observers are similar.

Table 13. The mean and standard deviations of the length difference and centerline deviation, and $\mathrm{p}$-values for the two observers.

\begin{tabular}{|l|l|l|l|l|l|l|}
\hline & \multicolumn{3}{|c|}{$\phi$} & \multicolumn{3}{c|}{$\varphi$} \\
\cline { 1 - 5 } & Mean & Std & p-value & Mean & Stct & p-walue \\
\cline { 1 - 6 } Observer 1 & 0.0192 & 0.0244 & 0.5905 & 1.5705 & 0.1355 & 0.7518 \\
\cline { 1 - 3 } Observer 2 & 0.0163 & 0.0183 & & 1.5798 & 0.1405 & \\
\hline
\end{tabular}

Table 14. The Pearson linear correlation coefficients between the results generated from the algorithm, the observer 1 , and the observer 2 .

\begin{tabular}{|l|l|l|l|l|l|l|}
\hline & \multicolumn{3}{|c|}{$\phi$} & \multicolumn{3}{c|}{$\varphi$} \\
\hline & Algarithm & Observer1 & Observer 2 & Algorithm & Observer1 & Observer2 \\
\hline Algarithm & - & 0.9946 & 0.9976 & - & - & - \\
\hline Observer 1 & 0.9946 & - & 0.9980 & - & - & 0.9053 \\
\hline Observer 2 & 0.9976 & 0.9980 & - & - & 0.9053 & N/A \\
\hline
\end{tabular}


Figure 16 (a)-(c) displays the quantile-quantile plots of the length and the centerline deviation of the neurite segment observed by the two individuals. In the plots, the symbol "+" represents the length values or the deviation values. A green line joints the first and third quartiles of each distribution, which indicates that the two sets of quantitative values have a linear fit of the order statistics. The red line is the extension of the green line to the ends of the data set to help evaluate the linearity of the data. Figure 16 (d)-(f) shows the cumulative distributions of the length and the center-line deviation of the neurite segment observed by the two individuals. All the plots prove that the quantitative results obtained from the algorithm, and the two observers have the same or similar statistics distributions.

The test neuron images are also manually traced by two users in such a way that the manual labels are completely independent and blinded to the automated results. The Quantilequantile plots and cumulative probability plots are displayed in Figure 17. From the figure we can see that the new line difference and centerline deviation are relatively larger than those if the manual results are guided by the automated results. However, the previous conclusions still hold. That is, the quantitative results from the two observers are still very similar. The quantitative results obtained from the algorithm, and the two observers have similar statistics distributions. The cumulative probability plots show that the independent manual results always have smaller line lengths compared to the automated results. This is due to the reason that the users always ignore some neurite centerlines, especially the weak lines. 


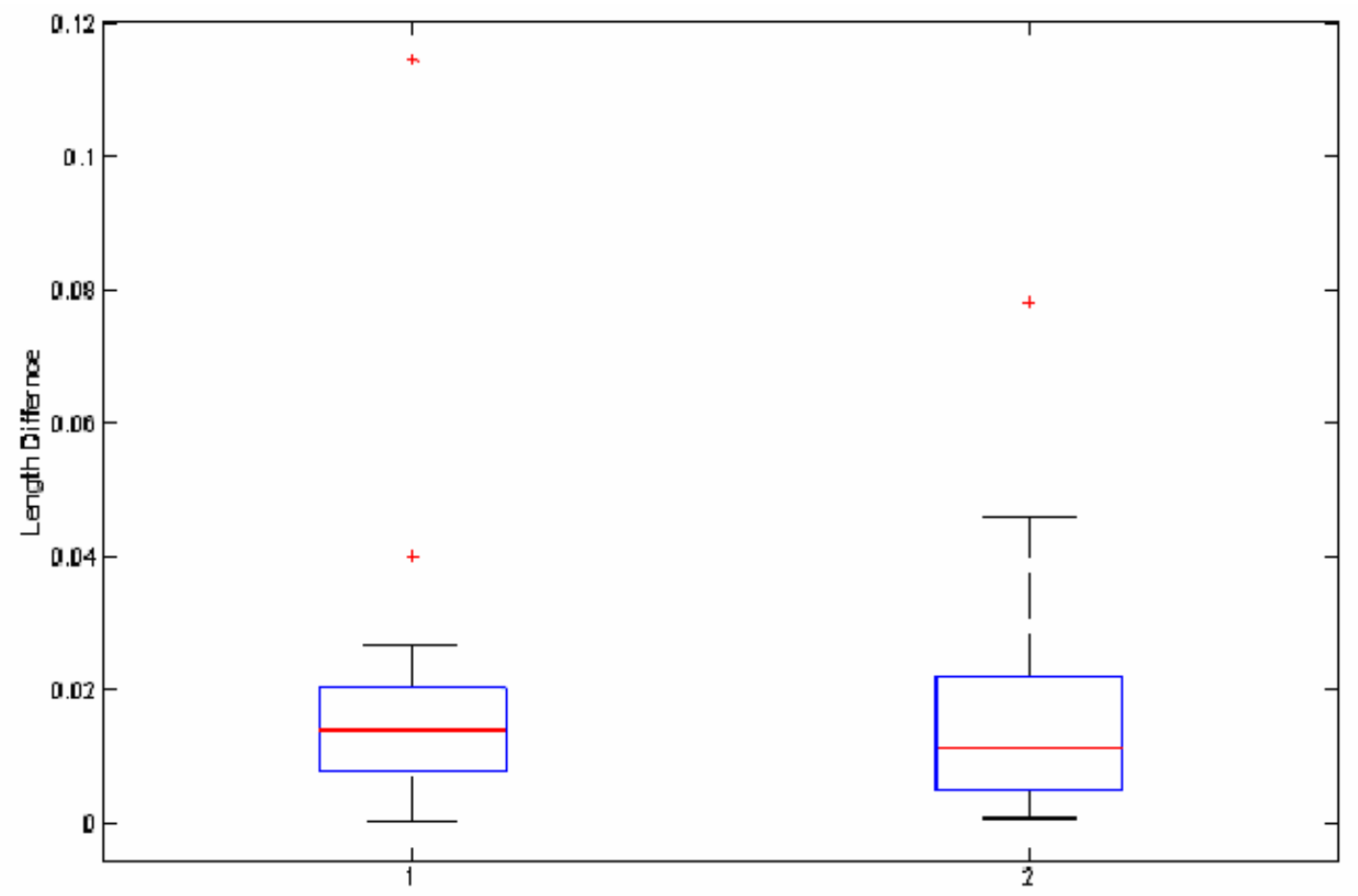

(a)

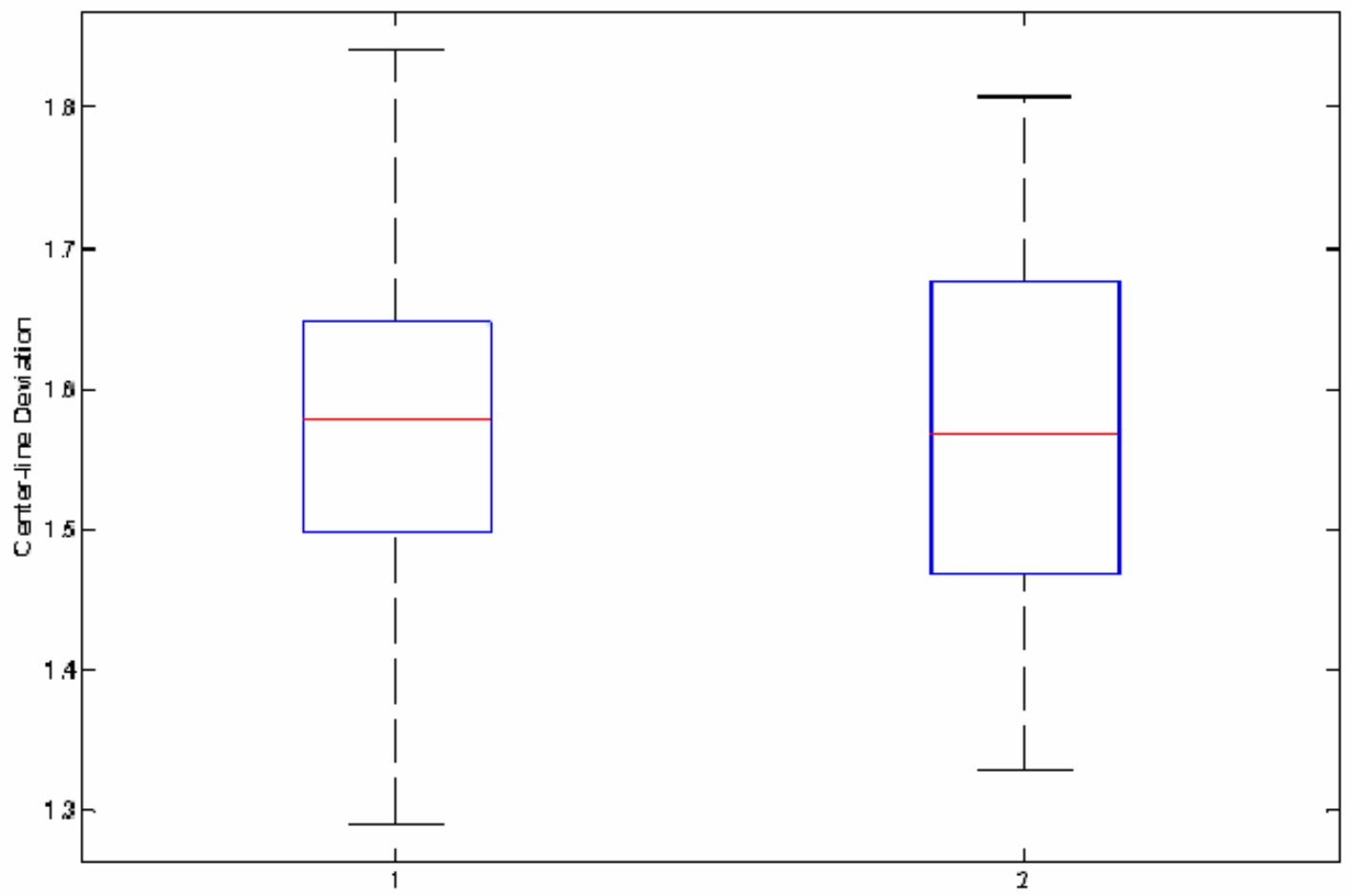

(b)

Figure 15. Box plots for the length difference and the centerline deviation. The red line is the mean value for each data set. The upper line of the box is the upper quartile value. The lower line of the box is the lower quartile value. (a) the length difference of the two observers; (b) the centerline deviation of the two observers. 

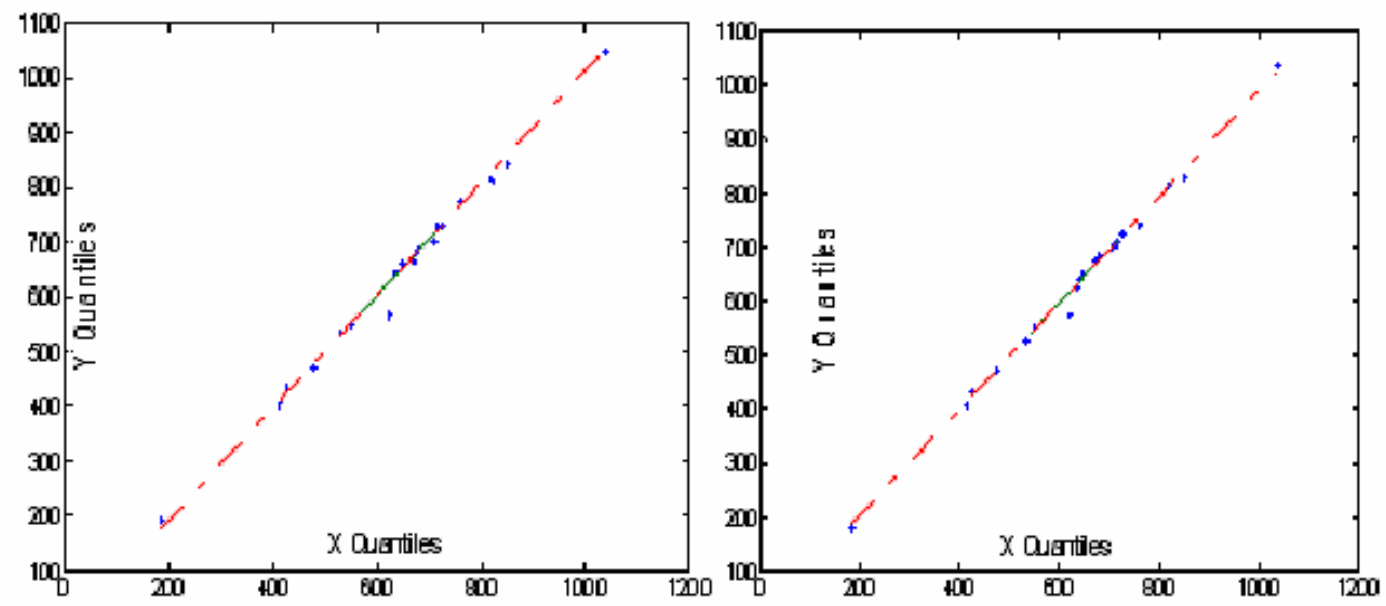

(a)
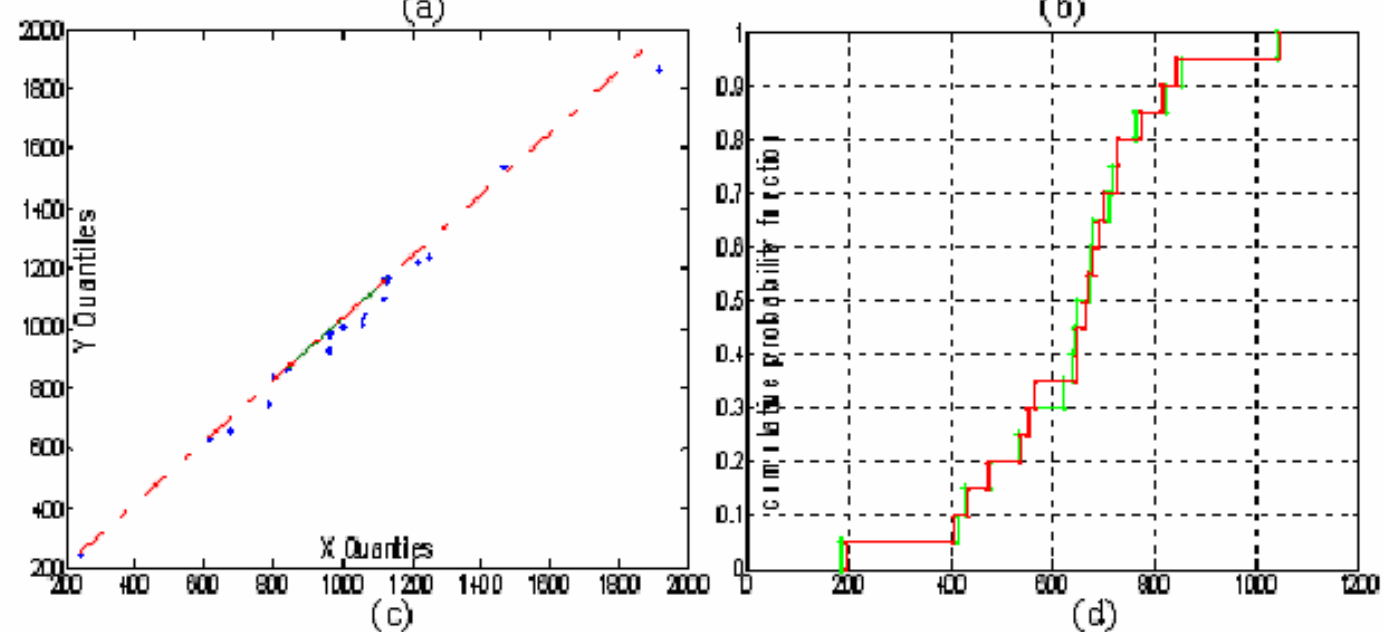

(C)
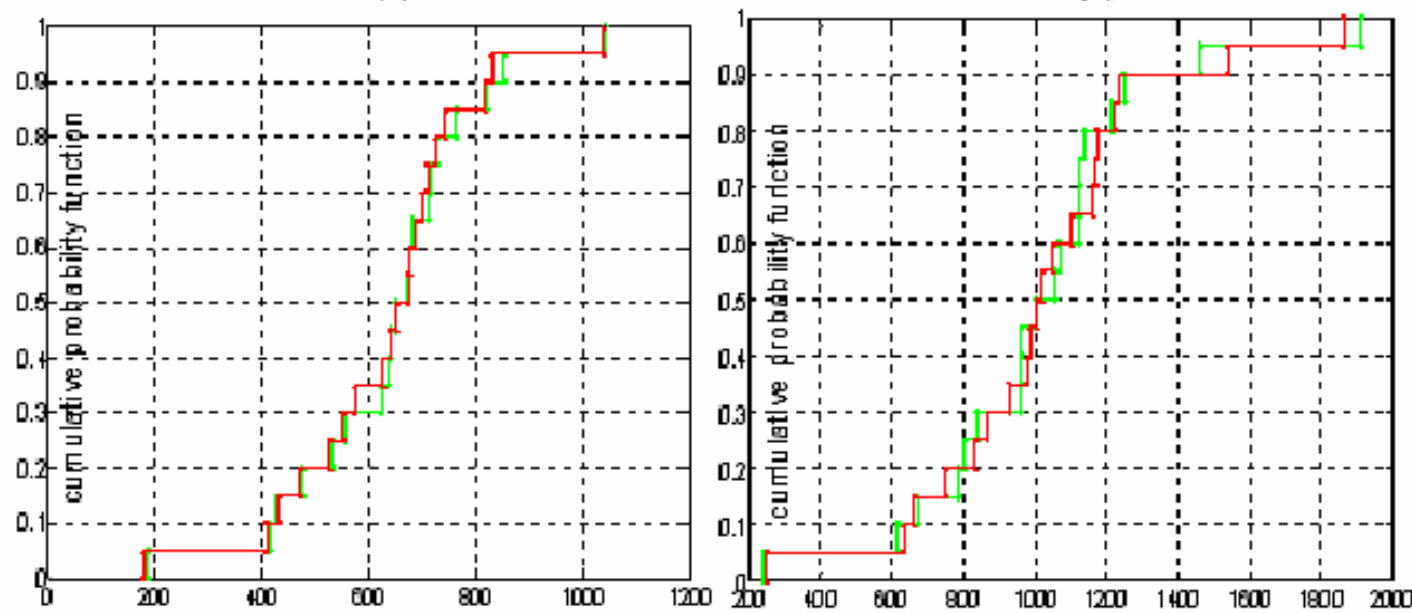

(e)

(f)

Figure 16. Quantile-quantile plots of the length of the neurite segments for (a) algorithm vs. observer 1; (b) algorithm vs. observer 2. Quantile-quantile plots of the centerline deviation for (c) observer 1 vs. observer 2. Cumulative distributions of the length of the neurite segments for (c) algorithm (green) vs. observer 1 (red); (d) algorithm (green) vs. observer 2 (red). Cumulative distribution of the centerline deviation for (e) observer 1 (green) vs. observer 2 (red). 


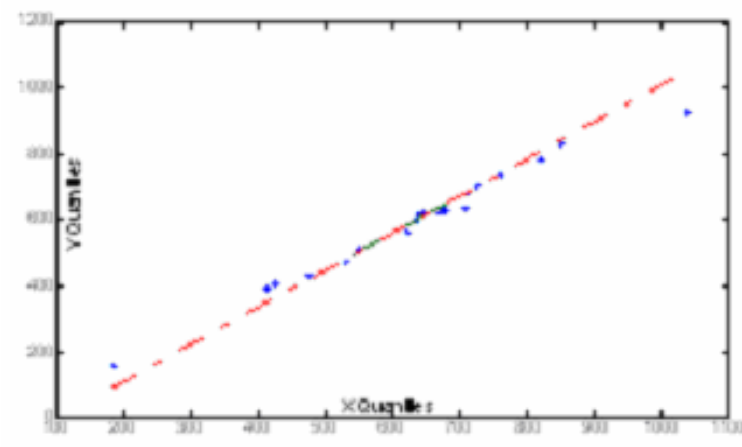

(a)

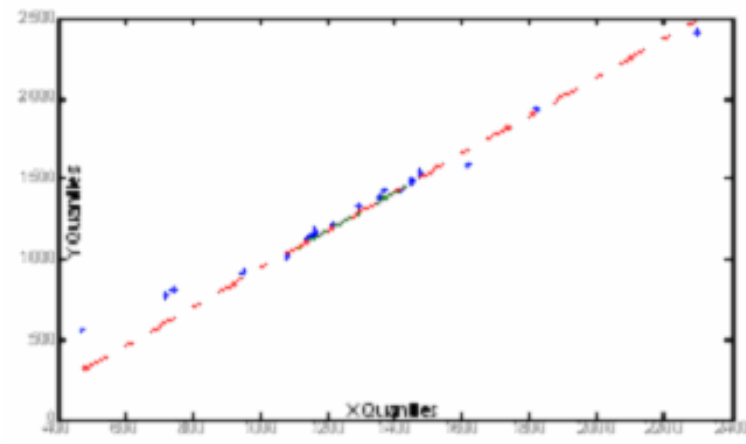

(c)

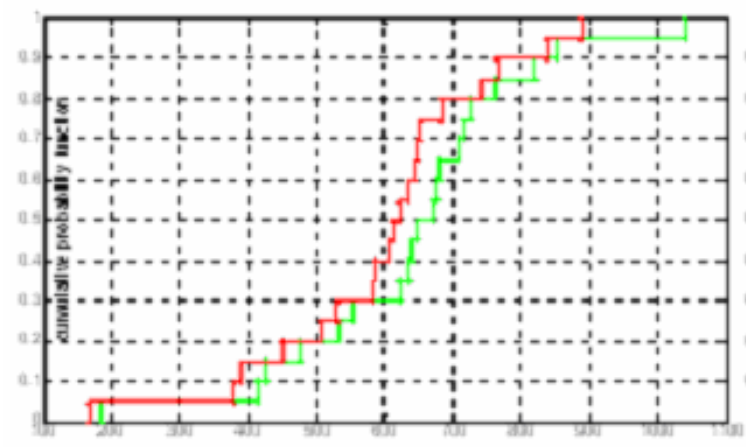

(e)

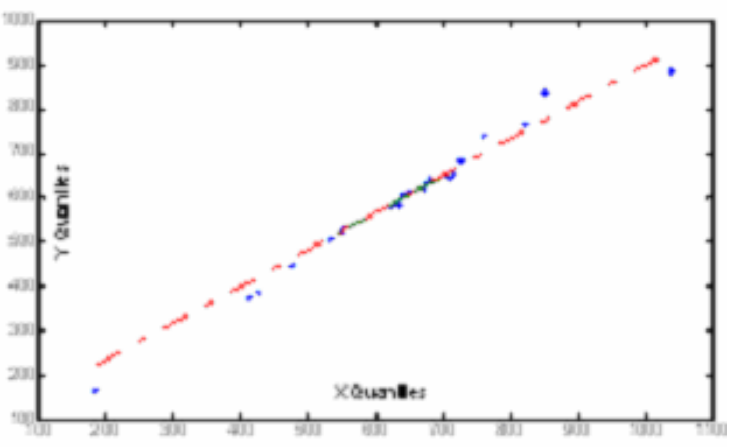

(b)

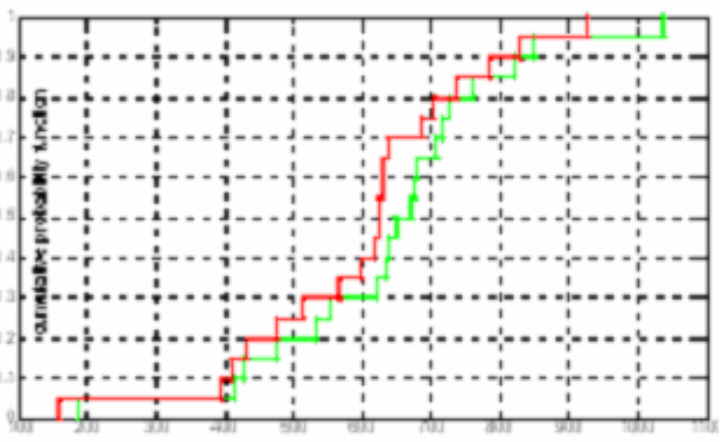

(n)

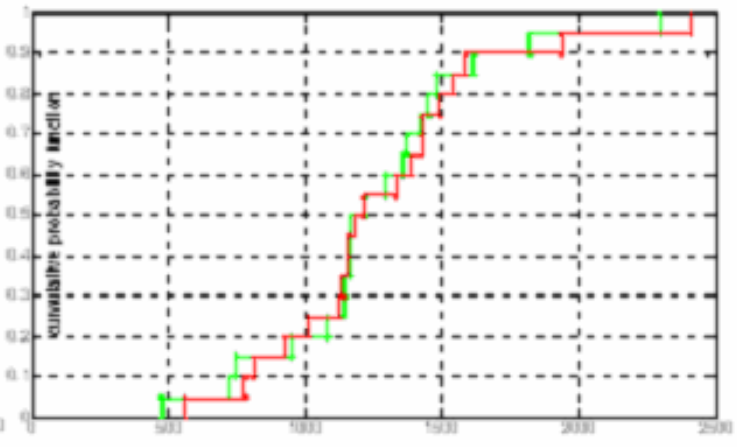

(f)

Figure 17. Quantile-quantile plots of the length of the neurite segments for (a) algorithm vs. observer 1; (b) algorithm vs. observer 2. Quantile-quantile plots of the centerline deviation for (c) observer 1 vs. observer 2. Cumulative distributions of the length of the neurite segments for (c) algorithm (green) vs. observer 1 (red); (d) algorithm (green) vs. observer 2 (red). Cumulative distribution of the centerline deviation for (e) observer 1 (green) vs. observer 2 (red).

\subsubsection{Parameter selection}

The parameters that may affect the extraction of neurite segments include maximum

possible width $W_{\max }$ of the neurite segments, the standard deviation $\sigma$ of the Gaussian kernel, 
and the weight $\varepsilon$ for the cost function. We explain in the following how these parameters may affect the performance of the algorithm.

The maximum possible width $W_{\max }$ is used when detecting the ending point of a neurite segment. It defines the searching range when detecting the edge points by examining the maximum template responses. In high-throughput applications, $W_{\max }$ can be determined by looking into a single neuron image, and then it can be used for all the other neuron images acquired from the same experiment. In this paper, we use $W_{\max }=12$ for all the 100 neuron images and the results are satisfactory.

The second-order derivative of the neuron image is estimated as the convolution of the image and the second-order derivative of a 2-D Gaussian kernel. The standard deviation $\sigma$ of the kernel is then an important factor that determines whether or not the centerline of a neurite segment can be detected correctly. In [2], it was shown that the detectable line structures have a width between 0 and $2.5 \sigma$, if the second-order derivative of the image is used to detect the lines. In this paper, the second-order derivative of a neuron image is used in the cost function (see formula 46) to determine the probability of a pixel being on the neurite segment. Thus a lot of neurite segments with relatively large width will be ignored by using a small $\sigma$. If $\sigma$ is selected large enough to cover most neurite segments, narrow segments with low intensity contrast will be smoothed out and parallel lines will affect each other. For example, in Figure 18 two extraction results are shown when using different $\sigma$. Obviously when $\sigma=6.5$, the extracted center line is influenced by neighboring lines and the extraction is not satisfactory. In this paper, we use $\sigma=2.0$ as a fixed value for all the neuron images from the same experiment. We believe that $\sigma$ can be determined adaptively based on each neurite segment and its second-order derivatives. We will consider the automatic selection of $\sigma$ in our future work. 
The weight $\varepsilon$ is used in the cost function defined in formula (46) to balance the influences of the two cost components. The local cost $c_{L}(p)$ of a point $p$ determines how well the extracted line approximates the centerline of the neurite segment. The linking cost $c_{D}\left(p_{1}, p_{2}\right)$ of two points determines the best way to link the points on the centerline by examining the similarity between their gradient directions. Figure 19 compares two extractions when using weight 1 and 0 respectively. The results show that the centerline of a neurite segment may not be extracted accurately if the local cost is not considered. In Figure 20 we compare the extraction performance between the situation when only local cost is considered and the situation when both the local cost and the linking cost are considered. The results prove that, even though the centerline can be extracted in both cases, the linking between the centerline points is usually smoother and tighter to a strong edge if the linking cost is considered. In this paper, we use $\varepsilon=0.8$ for all the images.

\subsection{Conclusion}

In this chapter we present a novel algorithm for extraction of neurite structures in $2 \mathrm{D}$ fluorescence microscopy neuron images. This algorithm is specifically designed for highthroughput screening of neuron-based assays, which usually requires the process to be fast and automatic. The algorithm is able to automatically select a pair of starting point and ending point for each neurite segment so that only minimum user interaction is required. The starting point is selected by detecting the local maxima after low-pass filtering a grid of lines in the image, followed by signal-to-noise ratio analysis to filter robust points. The automatic selection of the ending point is achieved by iteratively tracing the points on or near the centerline of the neurite segment until the end of the neurite structure. Robust starting points and redundant ending points are used so that the extraction is complete. Dynamic 
programming is used to link the points so that the centerline can be extracted rapidly, accurately, and smoothly. The results and validation indicate that our proposed algorithm can automatically and accurately extract most of the neurite segments in the image with complicated neurite structures. The algorithm is very efficient in applying the dynamic searching only on a small area of the image. All these advantages make the proposed algorithm suitable for the increasingly demanding and complex image analysis tasks in highthroughput neurite imaging applications and drug screening. 


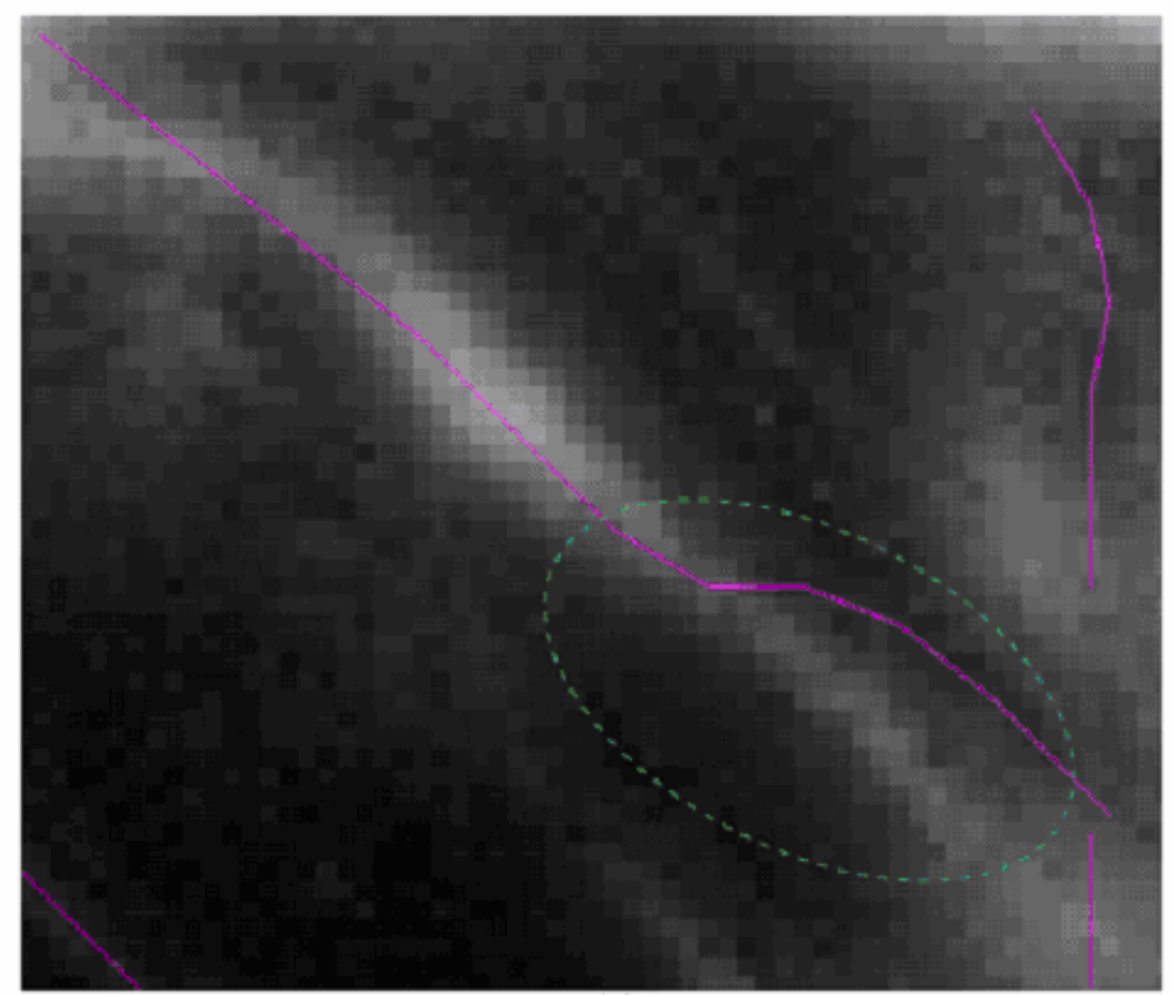

(a)

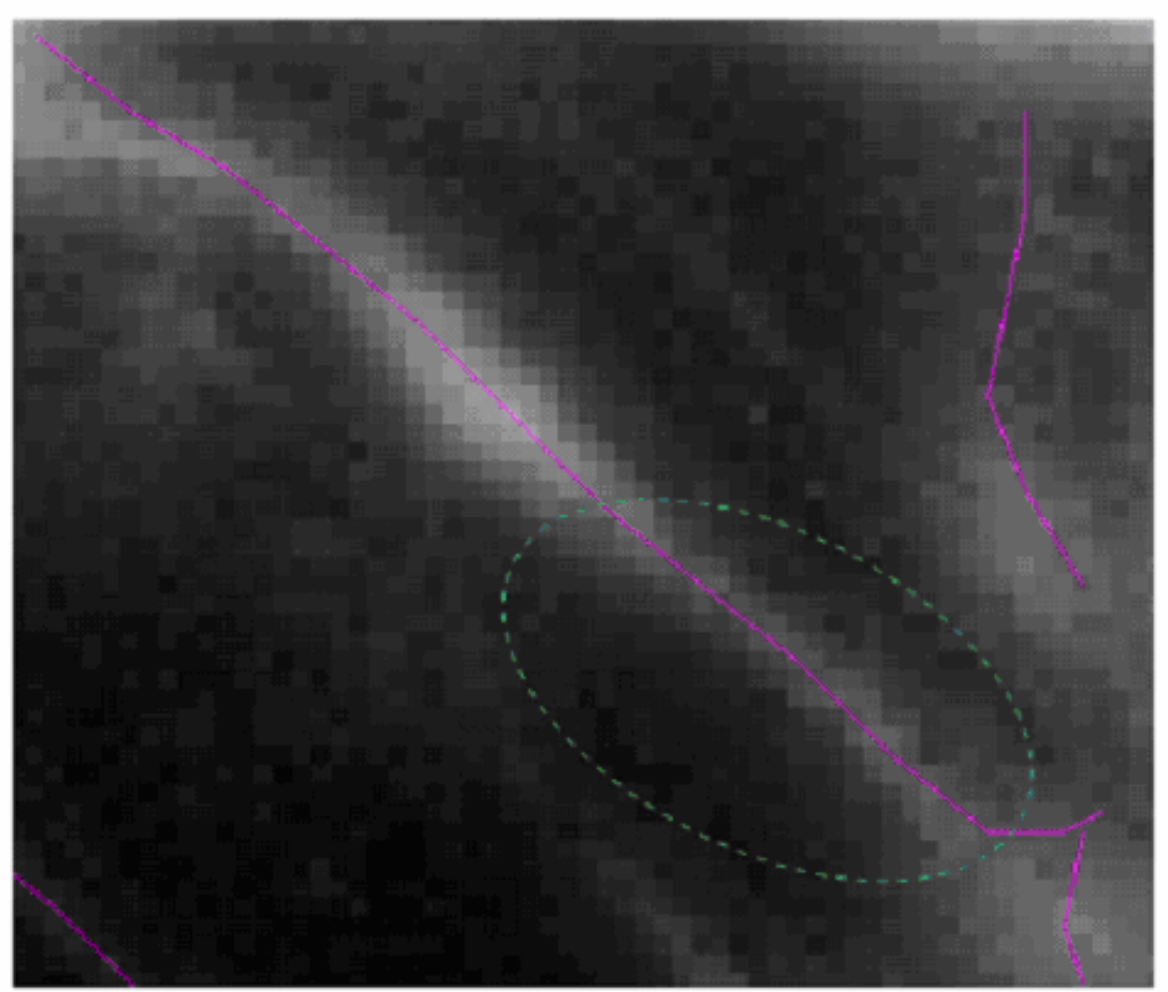

(b)

Figure 18. Comparison between extractions using different standard deviation (STD) values for the Gaussian kernel. A partial image is displayed with extracted centerlines labeled in color. Note the difference of the lines within the two dashed green circles. (a) $\mathrm{STD}=6.5$; (b) $\mathrm{STD}=2.0$ 


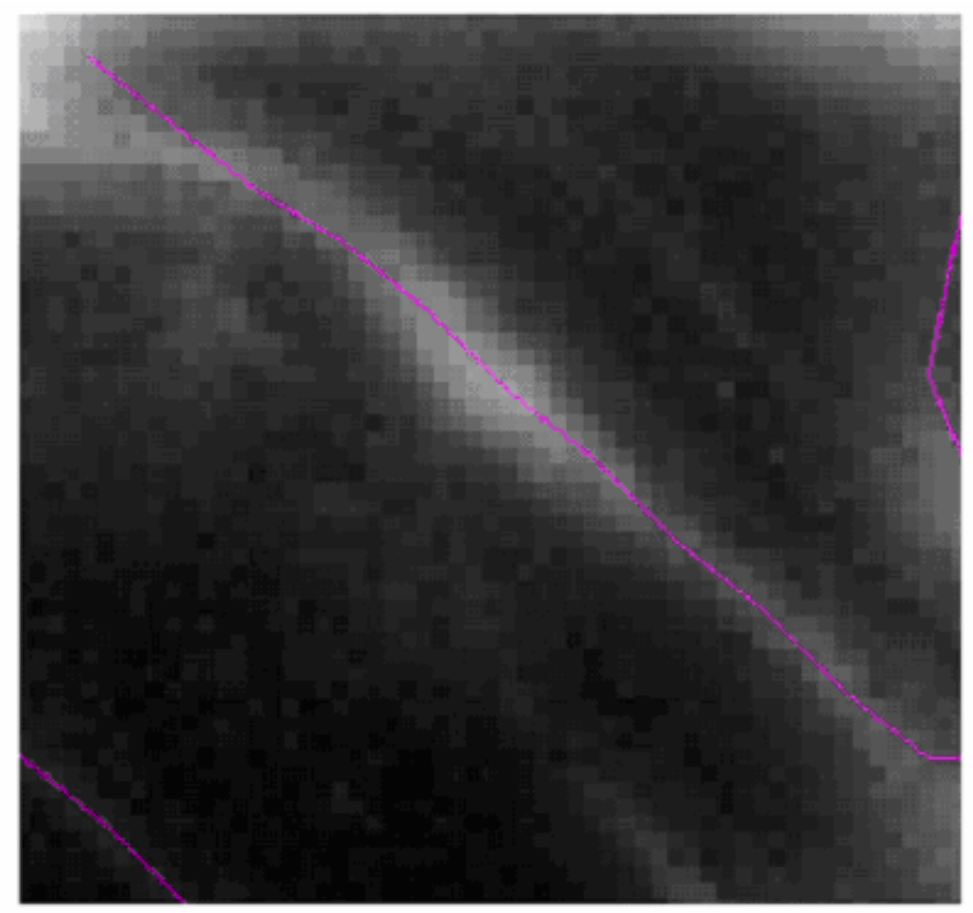

(a)

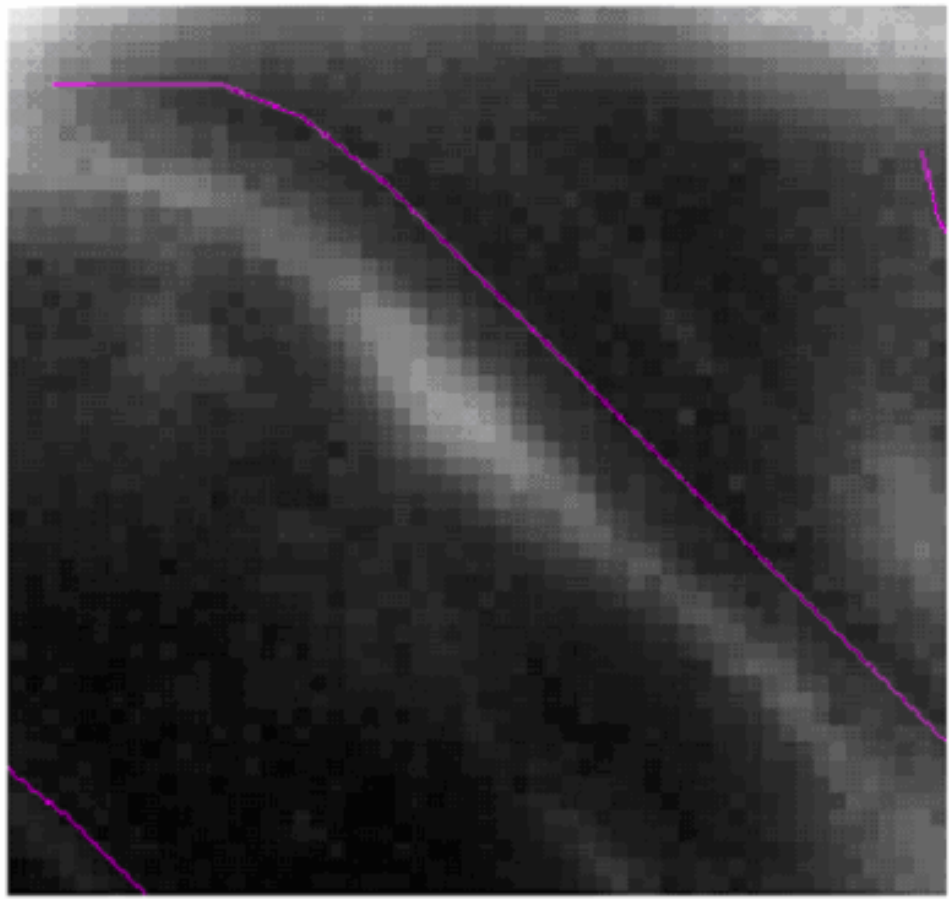

(b)

Figure 19. Comparison between extractions using different weight values $(w)$ for the cost function. A partial image is displayed with extracted centerlines labeled in color. Note the difference of the lines in the middle of the image. (a) $\varepsilon=1.0$; (b) $\varepsilon=0.0$. 


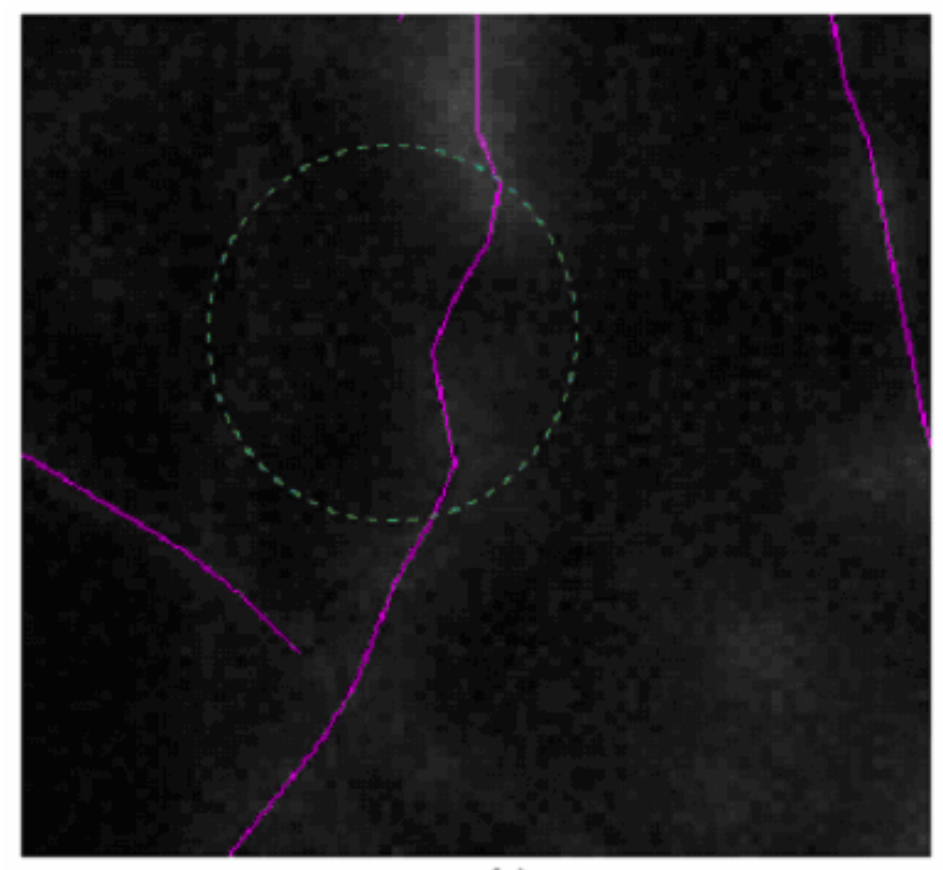

(a)

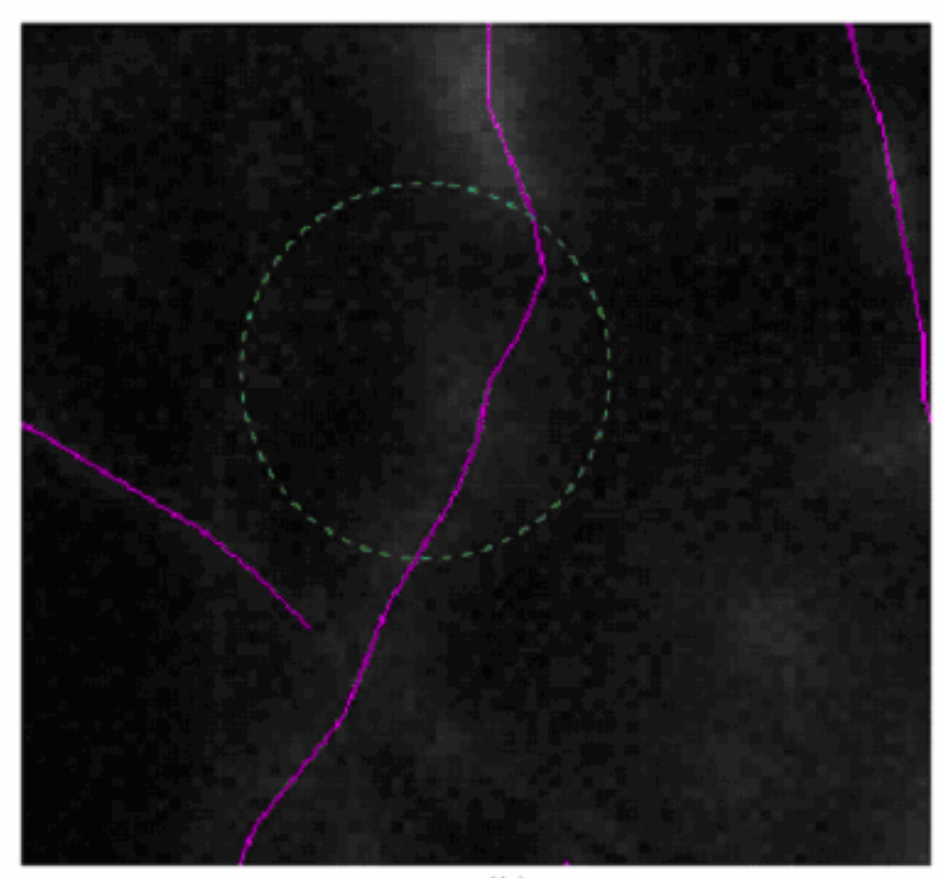

(b)

Figure 20. Comparison between extractions using different weight values $(\varepsilon)$ for the cost function. A partial image is displayed with extracted centerlines labeled in color. Note the difference of the lines within the two dashed green circles. (a) $\varepsilon=1.0$; (b) $\varepsilon=0.8$ 


\section{Chapter 4: Dendritic Spine Detection}

Related papers by Yong Zhang for this Chapter:

(1) Y. Zhang, X. Zhou, R. M. Witt, B. L. Sabatini, D. Adjeroh, and S. T. C. Wong, "Dendritic spine detection using curvilinear structure detector and LDA classifier," NeuroImage, under review, 2006. 


\subsection{Introduction}

The morphological and statistical changes of small cellular compartments, such as the dendritic spines, are of great interest when studying neurons [76-79]. As an example, Tavazoie et al [76] and Zhou et al [79] show that the TSC pathway regulates soma size, the density and size of dendritic spines, and the properties of excitatory synapses in hippocampal pyramidal neurons. Spine $\mathrm{Ca} 2+$ has a crucial role in the induction of most forms of synaptic long-term potentiation (LTP) and long-term depression (LTD) - the putative cellular mechanisms of learning and memory [80]. The inability to examine and measure fluorescent signals from individual spines or synapses has been a major obstacle for such study. Recently the 2-photon laser scanning microscopy (2PLSM) [81-83] has been widely used to image very small subcellular compartments within brain slices or in vivo, making it possible to study biochemical signaling that occurs at the intracellular level.

Despite previous work on automating the detection of dendritic backbones and spines $[43,84-86]$, the problem still remains largely unsolved, partially due to the irregular and diverse shapes of the objects. Herzog et al [84] proposed a method for 3D reconstruction of dendritic trees using a parametric model of cylinders. This method, however, missed most of the short spines or spines with thin neck. Al-Kofahi et al [43] use a generalized cylinder model to estimate the local directions and the dendritic backbones are directly traced from a set of pre-estimated seed points. The extraction of backbones is not complete due to the incomplete estimation of the seed points. Koh et al [85] construct a modified medial axis to locate dendritic backbones and detect spines as geometric surface protrusions relative to the backbones. Since the segmentation is achieved by a simple global thresholding method, and very limited geometric information is considered for spine detection, this method detects a lot of pseudo spines. 
In this paper, we propose a novel method for automated detection of dendritic backbones and spines suitable for 3D neuron images in thin slices. Fig. 4.1 shows a sample neuron image with dimension $512 * 512 * 11$. The $3 \mathrm{D}$ image stack is projected onto the $\mathrm{xy}, \mathrm{yz}$, and zx planes, respectively. Since the slices along the optical direction (z) provide very limited information, it is desired to consider only the 2D projection onto the xy plane. Our method treats the dendritic backbones and spines as curvilinear structures and extracts their centerlines and boundaries by estimating the second order directional derivatives. A classifier is built from a pre-selected training set and the detected spines are further classified to remove pseudo spines. Once the image stack is processed on its $2 \mathrm{D}$ projection, the very small number of missing spines can be detected by simply looking through the image stack. This way, the process is significantly simplified and very fast. Although the volume information about spines is missing, most of the geometric and statistical information is reserved and adequate for further biological analysis.

Among the photosensitive devices in use today, the photomultiplier tube (PMT) is a versatile device that provides extremely high sensitivity and ultra-fast response. However, the electronic signal converted by PMT detectors is usually noisy. Thus, image denoising is a necessary step before any further processing. Some methods for image denoising and restoration are discussed in [87]. In our case, the noise is mainly "salt and pepper" type. So we apply 2D median filtering [88] to remove the noise and preserve the boundary information for dendrites and spines. We treat the dendritic backbones and spines as curvilinear structures and extract their centerlines using the curvilinear structure detector [2]. The backbones and spines can be easily distinguished by examining the length of the detected centerlines, based on the observation that the length of a spine is much smaller than that of a backbone. The boundaries of the backbones and spines are extracted using information provided by the curvilinear structure detector for a fast process. Once the spines are detected, they are 


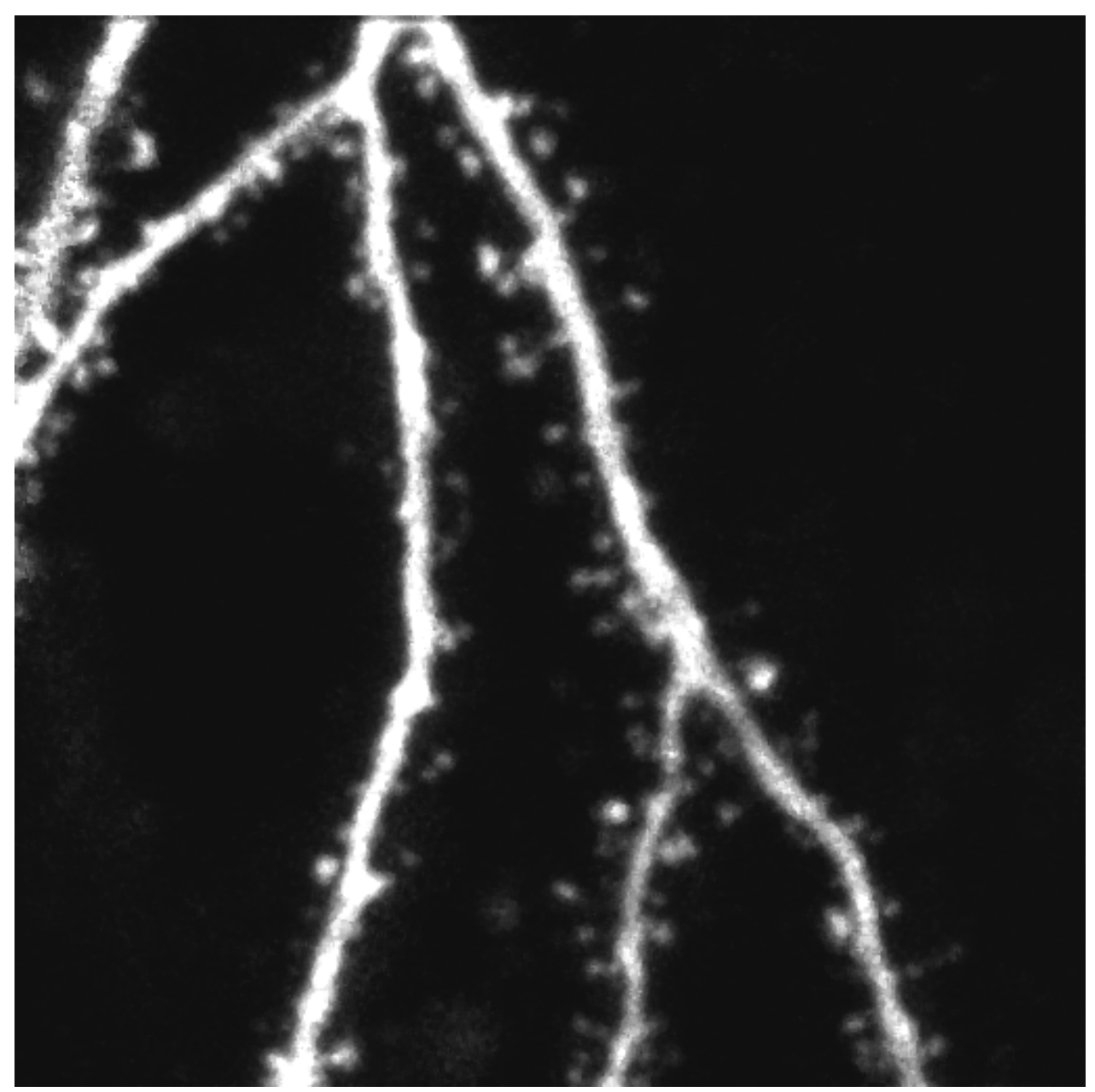

(a)

(c)

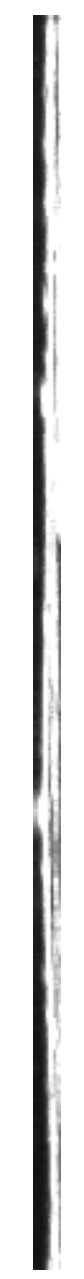

(b)

Figure 21 A sample neuron image stack projected along

(a) z-direction; (b) x-direction; and (c) y-direction.

grouped as either attached or detached based on the distance between the spine root and the backbone. For attached spines, we further classify them as spines or protrusions to improve the performance of the algorithm. In the final stage, the dendritic backbones and spines are quantified in terms of the spine number, backbone and spine length, and the spine density. Figure 22 shows the block diagram for our proposed approach.

The paper is organized as follows. Section 2 presents the dendritic backbone extraction method. The spine detection and classification are discussed in Section 3. The 
experimental results and validation are presented in Section 4. We conclude our work in Section 5 and necessary future work is discussed.

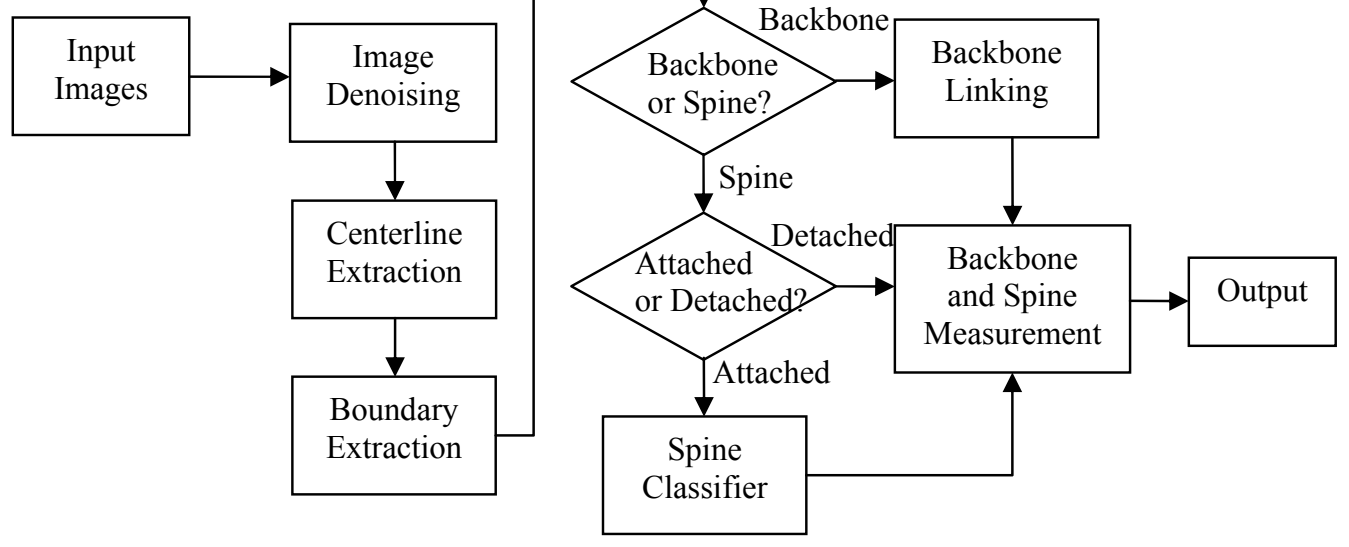

Figure 22 The block diagram for the automated backbone and spine detection

\subsection{Automated Extraction of Dendritic Backbones}

\subsubsection{Centerline Extraction for Dendritic Backbones}

A backbone is defined as the centerline of a dendrite segment. All the centerlines of the dendrite segments, if connected, form the dendritic backbone structure. The detection of the dendritic backbone involves extracting and connecting the centerlines and boundaries for all the dendrite segments. In this work, we apply the curvilinear structure detector $[2,89]$ to extract the centerlines of the dendritic backbone structures. Curvilinear structure detector is a robust line extraction method that is able to rapidly, accurately, and completely extract centerlines of line structures in 2D images. Basically, this method examines each pixel in the image and detects the line pixels that are located on or close to the line structures. These line pixels are then linked to form the centerlines of the line structures. A 2D line can be modeled as a curve that exhibits the characteristic $1 \mathrm{D}$ line profile in the direction perpendicular to the 
line, as shown in Figure 23. In our algorithm, there are five steps to extract the dendritic backbones:

- Step 1: local line direction estimation;

- Step 2: line point detection;

- Step 3: line point linking;

- Step 4: boundary extraction;

- Step 5: backbone linking.

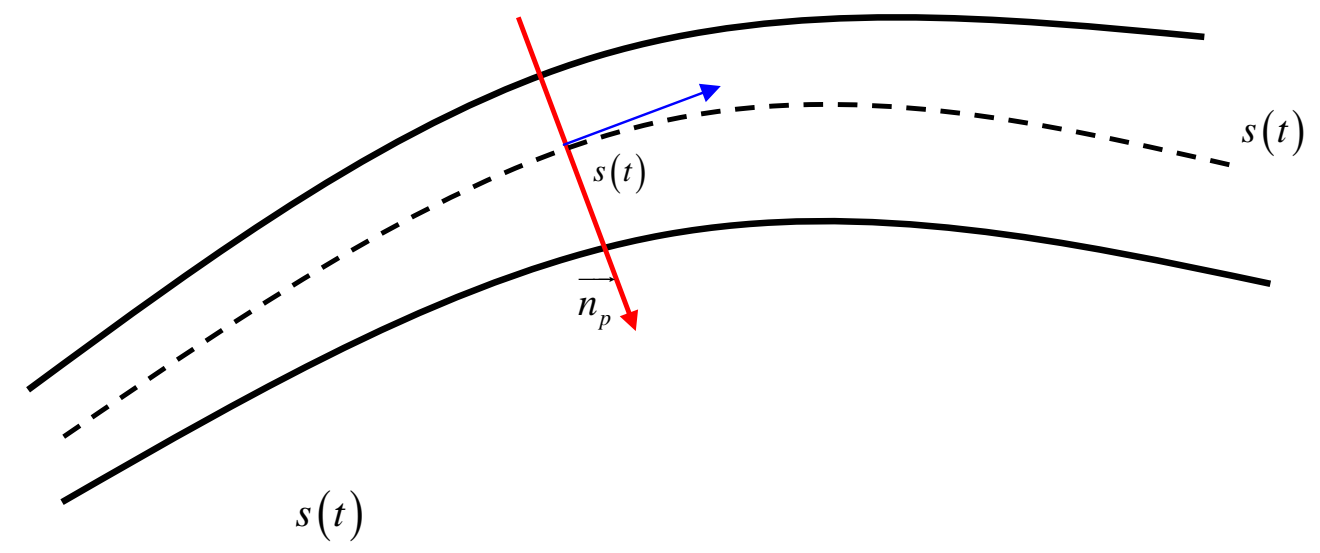

Figure 23 The 2D line model represented by a 1D line profile along the direction $\overrightarrow{n_{p}}$ perpendicular to the local line direction

The local direction of the line is determined using the second order directional derivatives of the image. However, since an image contains noise, the calculation of its derivatives is not straightforward. It is appropriate to estimate the derivatives of an image by convoluting it with the derivatives of the Gaussian kernel [59]. Thus, the derivatives of a noisy image $I$ can be estimated as follows: 


$$
\begin{aligned}
& r^{\prime}=(I * G)^{\prime}=I^{*} G^{\prime} \\
& r^{\prime \prime}=(I * G)^{\prime \prime}=I^{*} G^{\prime \prime}
\end{aligned}
$$

where " $G$ " represents the Gaussian kernel and "*”" is the convolution operator. The estimation of the derivatives for a 2D image can be achieved by convolving the image with the derivatives of the Gaussian kernel along one dimension and then convolving along the other dimension. After convoluting with the Gaussian kernel, the criterion for a bright salient line on a dark background is that the second-order derivatives $(r ")$ are much smaller than zero at locations where the first-order derivatives $\left(r^{\prime}\right)$ are equal to zero.

Since Gaussian kernel acts as a low-pass filter, it will blur the boundary information, or even smooth away weak line structures. Thus, the selection of the standard deviation $(\sigma)$ for the Gaussian kernel is restricted by two conditions:

1) $\sigma$ should be large enough so that $r^{\prime \prime}$ exhibit a clearly defined minimum at $r^{\prime}=0$;

2) $\sigma$ should not be too large to avoid smoothing away most of the line structures.

In order to preserve most of the line structures (including weak lines) during the convolution, we adopt the method in [89] to select multiple $\sigma$ values for different pixels in a neuronal image. Simply, we consider the Hessian matrix for each pixel and calculate different set of eigenvalues when using different $\sigma$ values for the convolution. We pick the $\sigma$ value such that its eigenvalue has the maximum absolute value among all the eigenvalues.

The eigenvector corresponding to the maximum absolute eigenvalue is the normalized direction perpendicular to the line. Denote this normalized direction as $\overrightarrow{n_{p}}$ where subscript $p$ represents the current pixel location. We call the second order derivative the strength value since it indicates how strong the line is. A noticeable line pixel must have a second order derivative that is larger than a threshold value, which is called strength threshold. $\overrightarrow{n_{p}}$ can 
also be represented as $\left(\overrightarrow{n_{x}}, \overrightarrow{n_{y}}\right)$, a unit vector along $\mathrm{x}$ and $\mathrm{y}$ directions. Once the local line direction is determined, the location of the candidate line point $(x, y)$ is given by:

$$
(x, y)=\left(s \overrightarrow{n_{x}}, s \overrightarrow{n_{y}}\right)
$$

where $s$ is calculated using the local line direction vector [89].

After the line points are detected, they are linked to form the centerline. The linking starts from the line points whose second directional derivatives are larger than the user-selectable threshold value, called upper-threshold. It ends at the line points whose second directional derivatives are lower than another user-selectable threshold value, called lower-threshold. When adding an appropriate neighbor to the current line, only three neighboring pixels that are compatible with the current line direction are examined. The appropriate neighbor is given by:

$$
p^{i+1}=\underset{k=1,2,3}{\arg \min }\left\{\left\|p^{i}-p_{k}^{i+1}\right\|_{2}+\left|\alpha^{i}-\alpha_{k}^{i+1}\right|\right\}
$$

where $p^{i}$ is the current line point and $p^{i+1}$ is the point being added to the line, $p_{k}^{i+1}$ $(k=1,2,3)$ are the three neighborhoods, $\alpha$ is the angle value given by: $\left(\overrightarrow{n_{x}}, \overrightarrow{n_{y}}\right)=(\cos \alpha, \sin \alpha)$.

\subsubsection{Boundary Extraction for Dendritic Backbones}

In [2] the boundaries of the curvilinear structures are extracted using a facet model line detector. In this paper we proposed a new method to accelerate the process. We consider only the bright lines on a dark background. The detection of the boundaries for such lines is based on the observation that the strength values of the line pixels change abruptly at the boundary locations. We perform boundary detection in three steps. In the first step, we examine the 
strength value for each pixel in the image and set it to zero if it is smaller than a pre-defined strength threshold. That is,

$$
S V(p)= \begin{cases}0 & \text { if } S_{p}<S T \\ S V(p) & \text { otherwise }\end{cases}
$$

where $S V(p)$ is the strength value for the pixel at location $p$ and $S T$ is the strength threshold value. The remaining pixels with non-zero strength values are then the line pixels noticeable in the foreground. We call it the strength image if each pixel is represented by its new strength value.

In the second step, starting from the first centerline point, we search the strength image along the two directions perpendicular to the local line direction until the strength value drops to zero. The stopping positions are then extracted as boundary points. The searching is applied to all the centerline points on each detected backbones. Each centerline pixel will have two boundary points associated with it. Let $d_{p}$ represent the local line direction for the current pixel $p$. The two perpendicular directions, if rotated to the left and to the right from $d_{p}$, are denoted as $d_{p}^{L}, d_{p}^{R}$ respectively. The points on the two searching directions are $P_{p}=\left\{p_{1}, p_{2}, \ldots, p_{K}\right\}$ and $Q_{p}=\left\{q_{1}, q_{2}, \ldots, q_{K}\right\}$, where $K$ is the maximum possible line width, Then the boundary points $b_{p}^{L}$ and $b_{p}^{R}$ for $p$ are:

$$
\begin{aligned}
& b_{p}^{L}=p_{i}, \text { if } p_{i} \in P_{p} \text { and } S V\left(p_{i+1}\right)=0 \\
& b_{p}^{R}=q_{j}, \text { if } q_{j} \in Q_{p} \text { and } S V\left(q_{j+1}\right)=0
\end{aligned}
$$

If the centerline points of a line structure is represented as $C=\left\{c_{1}, c_{2}, \ldots, c_{N}\right\}$, where $N$ is the total number of the points, $c_{1}$ is the starting point, and $c_{N}$ is the ending point of the line. For each point $c_{i}$, there will be two boundary points found by formula (52), then we find a set of 
boundary points for $C$ as $\left\{\left(b_{1}^{L}, b_{1}^{R}\right),\left(b_{2}^{L}, b_{2}^{R}\right), \ldots,\left(b_{N}^{L}, b_{N}^{R}\right)\right\}$. The search is very fast since it only involves finding the first zero-value point in the small sets of points $P_{p}, Q_{p}$.

Since only the boundary points on the two searching paths $d_{p}^{L}, d_{p}^{R}$ are found, other boundary points that are not on the paths may be missing. In the third step, the missing boundary points are detected from the neighboring boundary points. Given two known boundary points, if they are adjacent, i.e., there are no any other boundary points between them, then the Bresenham algorithm [90] is applied to find the points that can link them into a discrete line, with the two known boundary points as the starting and the ending points. These new points on the discrete line are regarded as the missing boundary points. That is, the complete boundary points for the centerline $C$ are:

$$
B=\left\{\left(b_{1}^{L}, b_{1}^{R}\right), l_{1,2}^{L}, l_{1,2}^{R},\left(b_{2}^{L}, b_{2}^{R}\right), l_{2,3}^{L}, l_{2,3}^{R}, \ldots,\left(b_{N}^{L}, b_{N}^{R}\right)\right\}
$$

where $l_{i, j}^{L}$ is the set of points on the discrete line that links point $b_{i}^{L}$ and $b_{j}^{L}, l_{i, j}^{R}$ for linking $b_{i}^{R}$ and $b_{j}^{R}$, Figure 24(a) shows an example results for the backbone boundary detection. We use different colors to represent the centerline points, boundary points, and the searching vectors.

The advantages of our proposed method for boundary detection are obvious: First, Our proposed method is fast. The linear-time searching of the boundary points is based on the second order derivatives and the local line directions obtained when extracting the centerlines, thus no further convolution and other computations are required. Second, the detected results are very accurate because of the use of the second-order derivatives, local line directions, and the removal of weak line pixels. Third, since each centerline pixel has two boundary points associated with it, this can be used to further detect the spine heads and roots, and also can be very useful for linking multiple backbones. 

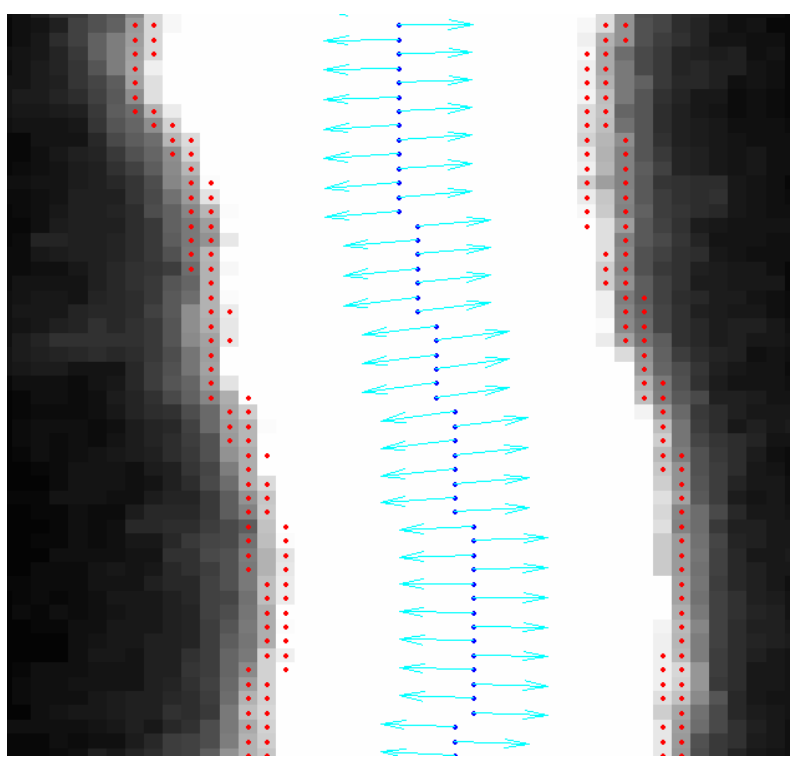

(a)

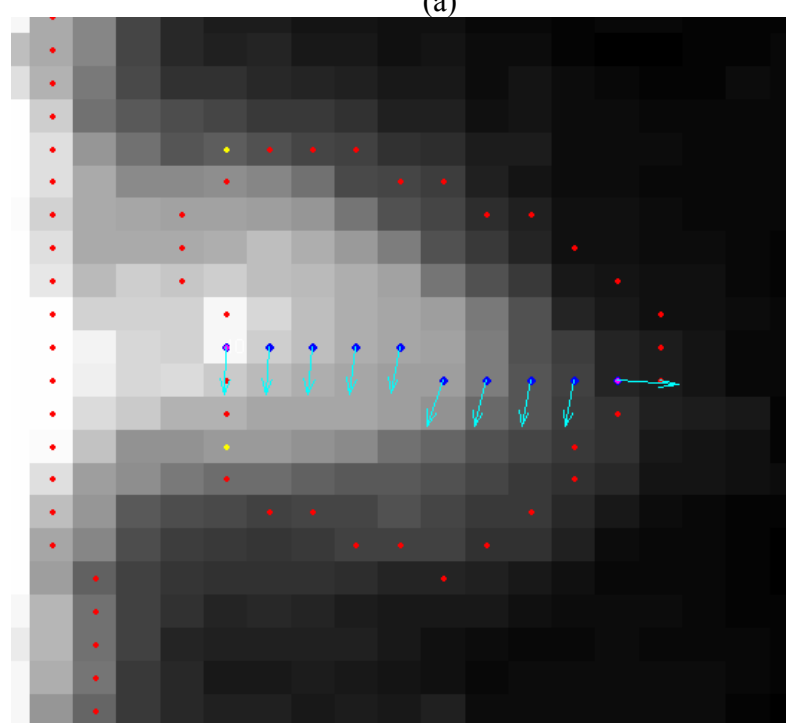

(b)

Figure 24 Example results for boundary detection. The vectors in cyan color are one of the two directions perpendicular to the local line direction. The blue points are the detected centerline points of the curvilinear structures. The red points are detected boundary points by searching along the vector directions and the opposite directions. Some boundary points are interpolated from the neighboring points. (a) backbone boundary detection results; (b) spine boundary detection results.

\subsubsection{Multiple Backbones Linking}

If more than one backbone are detected, they need to be linked together to construct the dendritic structures (see Figure 4.6 as an example). The backbones are linked based on their boundary information. If the boundary points for two backbones are overlapped or located 
within 8-neighborhood, they are considered belonging to the same dendritic structure and then the Bresenham algorithm [90] is used to directly link the two corresponding centerline points together. Otherwise the two backbones are said to belong to different dendritic structures and no linking is applied. If we denote one backbone $C^{U}$ and its boundary $B^{U}$ as two sets of points: $C^{U}=\left\{c_{1}^{U}, c_{2}^{U}, \ldots c_{M}^{U}\right\}, B^{U}=\left\{b_{1}^{U}, b_{2}^{U}, \ldots b_{X}^{U}\right\}$, another backbone and its boundary as $C^{V}=\left\{c_{1}^{V}, c_{2}^{V}, \ldots c_{N}^{V}\right\}, B^{V}=\left\{b_{1}^{V}, b_{2}^{V}, \ldots b_{Y}^{V}\right\}$, then the linking is applied if any one of the following four conditions is satisfied:

$$
\begin{aligned}
& \text { (1) if } b_{1}^{U} \text { or } b_{X}^{U}=b_{1}^{V} \text { or } b_{Y}^{V} \\
& \text { (2) if } b_{1}^{U} \text { or } b_{X}^{U} \in N_{8}\left(b_{1}^{V} \text { or } b_{Y}^{V}\right) \\
& \text { (3) if } b_{1}^{U} \text { or } b_{X}^{U}=b_{i}^{V}, b_{i}^{V} \in B^{V} \\
& \text { (4) if } b_{1}^{U} \text { or } b_{X}^{U} \in N_{8}\left(b_{i}^{V}\right), b_{i}^{V} \in B^{V}
\end{aligned}
$$

where $N_{8}(p)$ represents all the eight-neighborhood points of the pixel $p$. We link the two centerline points whose boundary points satisfy formula (54).

\subsection{Automated Spine Detection and Classification}

\subsubsection{Detached Spine Detection}

Dendritic spines are tiny membranous compartments consisting of a head (volume $\sim 0.01-1 \mu \mathrm{m}^{3}$ ) connected to the parent dendrite by a thin (diameter $\sim 0.1 \mu \mathrm{m}$ ) spine neck [91]. Once dendritic spines are detected, further analysis can be applied on morphological and statistical changes to study their activity patterns. Our algorithm extracts dendritic spines in four steps:

- Step 1: spine centerline extraction;

- Step 2: spine boundary extraction; 
- Step 3: spine root point detection;

- Step 4: spine classification;

The dendritic spines are treated as small protrusions of variable shape attached or detached to multiple dendritic backbones. We use the same method as for dendritic backbone extraction to extract the centerlines of dendritic spines. That is, the line points are detected and linked to form the centerlines for all the spine structures. The major difference, if compared with backbone extraction, is that the length of the centerline for a spine is much less than that for a backbone. Furthermore, we keep only those extracted spines if they are close enough to the nearby backbones. Otherwise, the detected objects are considered as noise or imaging artifacts. For spine boundary extraction, the method is exactly the same as that for detecting backbone boundary. However, we expect cyclic boundaries for spines. Thus, the boundary points are: $\left\{\left(b_{1}^{L}, b_{1}^{R}\right), l_{1,2}^{L}, l_{1,2}^{R},\left(b_{2}^{L}, b_{2}^{R}\right), l_{2,3}^{L}, l_{2,3}^{R}, \ldots,\left(b_{N}^{L}, b_{N}^{R}\right), l_{1,1}^{L, R}, l_{N, N}^{L, R}\right\}$, where $l_{1,1}^{L, R}, l_{N, N}^{L, R}$ means the two linking lines between $b_{1}^{L}$ and $b_{1}^{R}, b_{N}^{L}$ and $b_{N}^{R}$ respectively. Figure 24 shows an example result for spine boundary detection.

There are two types of spines: attached spine, which is attached to a backbone surface, and detached spine, which is detached but very close to a backbone surface. The method to distinguish them is as follows: the attached spines are those whose minimum distance to a backbone is no larger than the maximum width of that backbone. Otherwise they are detached spines. For attached spines, the edge of the spine intersects that of the backbone. We define the two intersecting points as the root points for that spine. The root points are very useful for calculating the spine features, such as length and width of the spine. Once the centerline and boundary of a spine is obtained, the root point detection is straightforward: we only need to consider the point on the centerline that is the closest to the backbone. The two corresponding edge points are then detected as the root points for that spine. Fig. 4.6 and Fig. 
4.7 show some example results for dendritic spine extraction. The centerline, boundary, and root points for each spine are labeled in colors.

\subsubsection{Attached Spine Detection}

The above described method is good enough for detecting detached spines. However, it usually detects some pseudo attached spines due to irregular shapes (protrusions) or noise around the boundary of the backbone areas. Here, we propose an automated attached spine selection procedure using feature selection and spine classification. The procedure is as follows: once the spines are extracted and grouped as attached and detached, we manually pick up a set of attached spines and categorize them as spine type or protrusion type. These categorized spines will be used as a training set for feature selection and spine classification. We calculate the geometric and moment features for each sample in the training set. We then select and normalize a subset of the features for further spine classification. Finally a classifier is applied to candidate objects to determine if they are spines or protrusions. We explain each step in the following subsections.

\subsubsection{Spine Feature Calculation}

Automated identification of the spine type relies on feature calculation, the most critical step in pattern recognition problems. Efficient features could improve the performance and reduce the complexity of the entire system. Considering the geometric and appearance properties of attached spines and pseudo spines, we use two types of features: Zernike moments and geometric features. The feature lengths are 36 for Zernike moments and 12 for geometric features. If a total of $m$ detected objects are manually selected as spine type $\left(s_{i}, i=1,2, \ldots, m\right)$ and a total of $n$ detected objects as protrusion type $\left(t_{j}, j=1,2, \ldots, n\right)$, then 
the training set $(T)$ can be represented as $T=\left\{s_{1}, s_{2}, \ldots, s_{m}, t_{1}, t_{2}, \ldots, t_{n}\right\}$. For each sample $s_{i}$ or $t_{j}$ in the training set, the calculated features are:

$$
\mathbf{F}=\left[F_{1}, F_{2}, \cdots, F_{m+n}\right]^{T} \text {, and } F_{i}=\left[z_{i 1}, z_{i 2}, \cdots, z_{i 36}, g_{i 1} g_{i 2}, \cdots, g_{i 12}\right], i=1: m+n
$$

where $z_{i j}, i=1: m+n, j=1: 36$ represents the Zernike moment features for the ith sample and $g_{i j}, i=1: m+n, j=1: 12$ represents the geometric features for the $i$ th sample. Thus, there are a total of $48 \times(m+n)$ features calculated for the training set. We briefly introduce these features below.

Zernike moments are classical image features that have wide applications [92, 93]. First, the center of mass is calculated for each spine polygon image and the spine pixels are redefined based on this center. Second, the radius is calculated for each spine, and the average of the radii is defined as $R$. Third, the pixel $(x, y)$ of the spine image is mapped to a unit circle to obtain the projected pixel $\left(x^{\prime}, y^{\prime}\right)$. Let $I\left(x^{\prime}, y^{\prime}\right)$ denote the florescence of the pixel. The Zerike moment for each cell is defined as:

$$
Z_{n l}=\frac{n+1}{\pi} \sum_{x} \sum_{y} V_{n l}^{*}\left(x^{\prime}, y^{\prime}\right) \cdot I\left(x^{\prime}, y^{\prime}\right)
$$

where $x^{\prime 2}+y^{\prime 2} \leq 1,0 \leq l \leq n, n-l$ is even, and $V_{n l}^{*}\left(x^{\prime}, y^{\prime}\right)$ can be calculated as:

$$
V_{n l}^{*}\left(x^{\prime}, y^{\prime}\right)=\sum_{m=0}^{(n-1) / 2}(-1)^{m} \frac{(n-m) !}{m !\left[\frac{n-2 m+l}{2}\right]\left[\frac{n-2 m-l}{2}\right]}\left(x^{\prime 2}+y^{\prime 2}\right)^{(n-2 m) / 2} e^{i l \theta}
$$

where $\theta=\arctan y / x$ and $i=\sqrt{-1}$. We select $n=10$ and use $\left|Z_{n l}\right|$ as the feature to obtain 36 moments features in total.

We also use a set of common region properties to describe the shape and texture characteristics (geometric features) of the spines in our work. For general texture description, 
we use the maximum, minimum, mean, and standard deviation of the intensity values in the segmented spine area. We also use some weak shape descriptions, such as the length of the

longest axis $l_{\max }$ and the length of the shortest axis $l_{\min }$, the ratio $l_{\max } / l_{\min }$, the spine area $S$, the spine perimeter $p$, and the spine compactness which is calculated as: compactness $=p^{2} /(4 \pi \cdot s)$. If the perimeter of minimum convex shape is $p_{c}$, then the spine roughness is: roughness $=p / p_{c}$. Finally, we have twelve general texture and shape features for each spine region.

\subsubsection{Spine Feature Selection}

The high-dimensional features bring two disadvantages in statistical analysis. First, the high-dimensional data with relatively sparse samples makes it difficult and inaccurate to parameterize the statistical model when training the classifiers. Second, the high-dimensional data require more computation cost, including time and memory space. Therefore, dimensionality reduction is a common way to condense the features. Feature-subset selection [94] and transformation based feature reduction are the two methods for reducing dimensionality of the feature space.

An optional choice is to apply the random search technique to derive an optimal feature subset. A genetic algorithm (GA) is a classical random optimization method, which mimics the evolutionary process of survival of the fittest [95]. Another popular method for feature selection is the Sequential Floating Forward Selection (SFFS) procedure [96]. This method starts with a single feature subset and iteratively adds or removes features until the performance is deteriorated. During the sequential search, once a forward step is applied, a number of backward steps are dynamically executed, given the condition that the performance can be improved. Even thought very simple, the SFFS algorithm has been shown to perform very well comparable to the GA method and even better for high- 
dimensional problems $[97,98]$. In our work, we apply the SFFS to the feature selection problem.

One important issue about feature selection is to determine the number of features that are appropriate for the classification problem. Because there is no criterion to determine this value, we have to try different number of features during feature selection. In practice, we select $8,10,12,14$, and 16 features and compare the performance (see Section 4.3). Once the features for the two classes are selected, they will be used to classify an unknown object into a spine or a protrusion.

\subsubsection{Attached Spine Classification}

There exist many classification methods in the literature, for example, the NearestNeighbor Classifiers (kNN), Support Vector Machines (SVM), Neural Networks, and Decision Trees. Most of these methods are either too simple or too complicated (computationally expensive) to be used in this project. We use the Linear Discriminate Analysis (LDA) as the classification algorithm in our work. LDA easily handles the case where the within-class frequencies are unequal and their performances have been examined on randomly generated test data. It is a transform-based method which attempts to maximize the ratio of between-class variance to the within-class variance in any particular data set, thereby guaranteeing maximal separability [99]. LDA is to find an optimal linear transformation, which can retain the class separablility while reducing the variation within each class. In the training set, suppose we have two feature sets for the two classes (spine $\mathbf{S}$ and protrusion $\mathbf{T}$ ) in the forms given below:

$$
\mathbf{S}=\left[S_{1}, S_{2}, \cdots, S_{m}\right]^{T}, \mathbf{T}=\left[T_{1}, T_{2}, \cdots, T_{n}\right]^{T}
$$

where $S_{i}=\left[s_{i 1}, s_{i 2}, \cdots, s_{i 10}\right], i=1: m$, and $T_{j}=\left[t_{j 1}, t_{j 2}, \cdots, t_{j 10}\right], j=1: n$. Let $\mu_{\mathrm{s}}, \mu_{\mathbf{T}}$ be the mean vector of the two sets respectively and $\mu$ be the mean vector of the entire training set. 
The between-class scatter matrix $\left(S_{b}\right)$ of the training set defines the scatter of the expected vectors around the global mean:

$$
S_{b}=\left(\boldsymbol{\mu}_{\mathbf{S}}-\boldsymbol{\mu}\right)\left(\boldsymbol{\mu}_{\mathbf{S}}-\boldsymbol{\mu}\right)^{T}+\left(\boldsymbol{\mu}_{\mathbf{T}}-\boldsymbol{\mu}\right)\left(\boldsymbol{\mu}_{\mathbf{T}}-\boldsymbol{\mu}\right)^{T}
$$

The within-class scatter matrix $\left(S_{w}\right)$ of the training set defines the scatter of samples around their respective means:

$$
S_{w}=E\left[\left(\mathbf{S}-\boldsymbol{\mu}_{\mathbf{S}}\right)\left(\mathbf{S}-\boldsymbol{\mu}_{\mathbf{S}}\right)^{T}\right]+E\left[\left(\mathbf{T}-\boldsymbol{\mu}_{\mathbf{T}}\right)\left(\mathbf{T}-\boldsymbol{\mu}_{\mathbf{T}}\right)^{T}\right]
$$

The transformation matrix is formed by combining $S_{b}$ and $S_{w}$ so as to maximize the separation of the classes in the transformed domain. In this work, the transformation matrix (H) consists of the eigenvectors corresponding to the dominant eigenvalues of the matrix $S_{w}^{-1} \times S_{b}$. Once $\mathbf{H}$ is obtained, the training data set $(\mathbf{S}, \mathbf{T})$ and the unknown data set $(\mathbf{U})$ are transformed to the new domain. A class is assigned to each unknown data vector in $\mathbf{U}$ based on the Euclidean distance between the transformed unknown data sample and each of the class centers in the transformed domain. Figure 25 shows the block diagram for the spine classification using the LDA method.

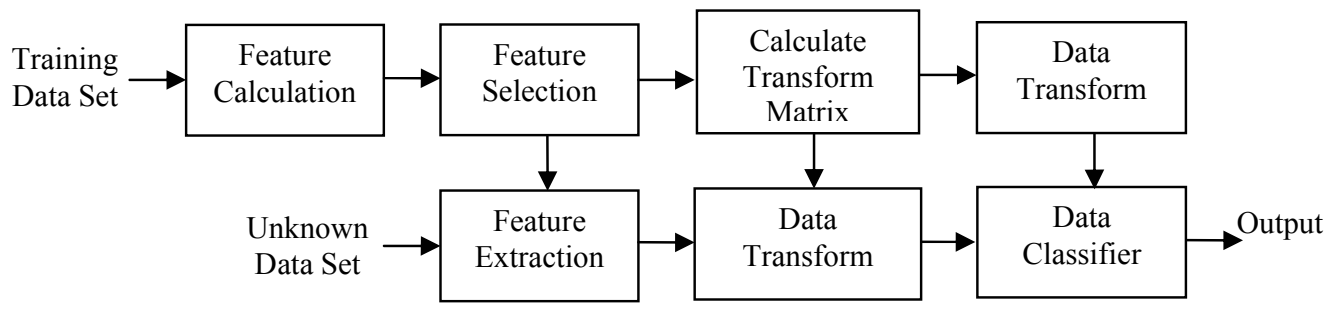

Figure 25 The block diagram for the spine classification using LDA 


\subsection{Experimental Results and Validation}

\subsubsection{Neuron Image Acquisition}

The test images are provided by Bernardo's Lab, one of our collaborators at the Department of Neurobiology, Harvard Medical School. The neuron images are acquired with two-photon laser scanning microscopes with an excitation wavelength of $910 \mathrm{~nm}$. Images of transfected pyramidal neurons are acquired at $0.8 \times$ zoom (image field, $300 \times 270 \mu \mathrm{m}$ ), whereas spiny regions of basal and apical dendrites are imaged at $5 \times$ magnification (image field, $42 \times 42 \mu \mathrm{m})$. Optical sections were taken at $1.0 \mu \mathrm{m}$ spacing.

\subsubsection{Results for Dendritic Backbone and Spine Extraction}

Figure 26 and Figure 27 show four original neuron images and their extracted backbones and spines labeled with color. Each image and its result represent one of the three situations: single backbone; multiple connected backbones; and multiple separated backbones. The results also show that, even with very noisy images, our proposed algorithm can accurately and robustly extract complicated backbone and spine structures. The boundaries for backbone and spines are also shown. The average running time to process one neuron image is in minutes, compared to time in hours spent if labeled manually. The results show that our method yields very accurate performance and is highly time efficient.

\subsubsection{Results for Spine Classification}

From 27 neuron images we pick 157 attached spine samples as valid spine type and 63 samples as protrusion type, for a total of 220 positive and negative spine samples in the training set. Figure 28 shows some sample spine images from the training set. We calculate 
the 12 geometric features and 36 moment features for each of the sample spine image. A $220 \times 48$ feature matrix is then generated with each row representing a single sample spine and each column a single feature. After the feature normalization, we examine the correlation between each pair of columns in the feature matrix by calculating the pairwise linear correlation coefficients. The correlated features are removed in order to make the feature matrix positive definite. After the feature decorrelation, only 22 features are left for feature selection and spine classification. The SFFS method is applied to the 22 uncorrelated features to select 8, 10, 12, 14, or 16 features for further classification. The Error Rate is defined as the proportion of errors made over the whole set of testing samples. The error rate values are obtained using the 10-fold cross-validation method. First, the whole sample spines are split into 10 subsets of equal size. Second, each subset is used in turn for testing, the remainder for training. Third, the SFFS method is applied and the error estimates are averaged to yield an overall error rate. In Table 15 we show the performance of SFFS in terms of error rates for different number of selected features. In this work we use 10 features for classification.

Table 15. SFFS feature selection performance for different number of features

\begin{tabular}{|c|c|c|c|c|c|}
\hline Feature Num & 8 & 10 & 12 & 14 & 16 \\
\hline Error Rate & $2.27 \%$ & $\mathbf{1 . 3 6 \%}$ & $\mathbf{1 . 3 6 \%}$ & $1.82 \%$ & $2.27 \%$ \\
\hline
\end{tabular}

We further test the feature selection method using a holdout procedure: From the 220 samples, we randomly select $80 \%$ of them (176 samples) as the training set and remaining $20 \%$ (44 samples) as the testing set. 10 features are selected by SFFS. The classification result shows that there are 2 errors out of 44 samples and the error rate is $4.55 \%$ ( 2 out of 44 ). That means, the spine classifier can successfully predict the spine type for $95.45 \%$ of the samples. 
Given the training set and its classification results, we apply LDA as the classifier to detect the spine type for unknown spines. That is, once an attached spine is detected, it is fed to the classifier as an unknown test data. The classifier will determine whether or not it belongs to the valid class, based on the predefined training data set and its classification results. Figure 29 and Figure 30 show the spine detection results before and after the spine classification. It is observed that some pseudo spines are removed by the classifier.

We compare our spine detection results with those produced by the medial axis and global threshold based algorithm [85]. Figure 31 and Figure 32 shows the comparison results for the four test neuron images. The medial axis and global threshold based method accepts many noisy spots as spines. Furthermore, some parts of the dendrites are incorrectly detected as spines (for example, see Figure 32 (a)(b)). Our proposed method is robust enough to bypass noise around valid spines and the dendritic backbones are detected very accurately.

\subsubsection{Validation}

In order to evaluate our proposed algorithm for dendritic backbone and spine extraction, we compare our results with those of semi-automatic method and fully-manual method by visual inspection and quantitative comparison. The validation process is designed as follows: we randomly select 14 neuron images from a total of 109 images. For each image we randomly select one dendrite region and its spine structures to alleviate the burden of manual process. We manually label the backbones and the centerlines of the spines in each selected dendrite and use the results as the ground truth. Our proposed algorithm is then applied on each dendrite and the results are compared quantitatively with the manual results in terms of spine number, spine length, backbone length, and spine density. The spine density is defined as the number of spines per unit backbone length.

The manual method detects 254 spines and the automated method detects 260 spines. Among these spines a total of 241 spines are detected by both methods, 13 additional spines 
are detected only by manual method, and 19 additional spines are detected only by automated method. Visual comparisons are demonstrated in Figure 33 and Figure 34. In Figure 33 the spines circled are detected only by manual method. Since these spines are due to the noise and the rough dendrite boundaries, the automated classifier treats them as invalid attached spines and removes them. Figure 34 shows two examples in which case the two methods obtain exactly the same number of spines. This proves the accuracy of our proposed method.

Table 16 compares the two methods quantitatively. The average spine length and spine density are calculated based on the 241 common spines. Table 17 compares the spine number, spine length, and spine density for each individual test image. The measured backbone length by the automated method is always larger than that by the manual method. This is partly due to the fact that the automated extracted centerline is a smoothed curve, while the manually extracted centerline consists of a set of straight lines, which is usually shorter than a curve. Both visual inspection and quantitative comparison show that the proposed method is accurate and complete for the automated detection of dendritic backbone and spines.

Table 16. Comparison of spine number and average spine length measured by two methods

\begin{tabular}{|c|c|c|c|c|}
\hline Method & $\begin{array}{c}\text { Spine } \\
\text { Number }\end{array}$ & $\begin{array}{c}\text { Average Backbone } \\
\text { Length (in micron) }\end{array}$ & $\begin{array}{c}\text { Average spine } \\
\text { Length (in micron) }\end{array}$ & $\begin{array}{c}\text { Spine Density } \\
\text { (in 1/micron) }\end{array}$ \\
\hline Manual & 254 & 49.133 & 1.147 & 0.35 \\
\hline Automated & 260 & 52.158 & 1.247 & 0.33 \\
\hline
\end{tabular}

\subsubsection{Parameter selection}

The standard deviation for the Gaussian kernel is determined automatically. Other parameters for the curvilinear structure detection, such as the lower and upper threshold, the minimum line length, and the linking distance, can be fixed loosely using a large range and does not affect the final results significantly. The parameters for the backbone and spine detection include the weakness threshold and the minimum backbone length. These two 
parameters may vary for different images, but can be fixed for a set of images from the same biological experiment, or images with similar intensity distributions. Table 18 lists the typical parameter values used in this work.

Table 17. Quantitative comparison of each individual test image

\begin{tabular}{|c|c|c|c|c|c|c|c|}
\hline \multirow{2}{*}{ Image } & \multicolumn{3}{|c|}{ Spine Number } & \multicolumn{2}{c|}{$\begin{array}{c}\text { Total Spine Length (241) } \\
\text { (pixel) }\end{array}$} & \multicolumn{2}{c|}{$\begin{array}{c}\text { Backbone Length } \\
\text { (micron) }\end{array}$} \\
\cline { 2 - 8 } & Manual & Auto & Both & Manual & Auto & Manual & Auto \\
\hline fd124b003 & 20 & 20 & 19 & 17.524 & 19.387 & 56.24 & 62.229 \\
\hline fd124b005 & 16 & 14 & 14 & 14.784 & 20.706 & 48.414 & 51.899 \\
\hline fd124b007 & 16 & 18 & 15 & 11.537 & 13.725 & 46.464 & 50.040 \\
\hline fd124c003 & 11 & 13 & 11 & 14.213 & 13.913 & 28.149 & 30.565 \\
\hline fd124e006 & 12 & 11 & 10 & 10.695 & 9.951 & 70.128 & 71.976 \\
\hline fd125f002 & 21 & 22 & 20 & 29.151 & 32.098 & 44.666 & 46.067 \\
\hline fd136c007 & 17 & 16 & 16 & 22.696 & 28.267 & 72.993 & 77.874 \\
\hline fd136d004 & 9 & 13 & 9 & 9.798 & 10.831 & 43.682 & 47.017 \\
\hline fd136e004 & 21 & 19 & 19 & 19.850 & 23.124 & 48.456 & 49.006 \\
\hline fd136f003 & 33 & 34 & 32 & 42.065 & 49.040 & 60.818 & 66.409 \\
\hline fd136f005 & 22 & 22 & 22 & 26.970 & 27.116 & 32.964 & 34.061 \\
\hline fd140f003 & 20 & 20 & 20 & 19.498 & 19.851 & 50.874 & 55.322 \\
\hline fd140f005 & 15 & 14 & 13 & 12.535 & 12.354 & 43.079 & 45.274 \\
\hline fd149d003 & 21 & 24 & 21 & 25.095 & 20.154 & 40.933 & 42.472 \\
\hline Total & $\mathbf{2 5 4}$ & $\mathbf{2 6 0}$ & $\mathbf{2 4 1}$ & $\mathbf{2 7 6 . 4 0 9}$ & $\mathbf{3 0 0 . 5 1 7}$ & $\mathbf{6 8 7 . 8 6}$ & $\mathbf{7 3 0 . 2 1 2 7}$ \\
\hline Average & & & & $\mathbf{1 . 1 4 7}$ & $\mathbf{1 . 2 4 7}$ & $\mathbf{4 9 . 1 3 3}$ & $\mathbf{5 2 . 1 5 8}$ \\
\hline
\end{tabular}

Table 18. Typical parameter values

\begin{tabular}{|c|c|c|}
\hline Parameter & Typical value & Description \\
\hline Upper threshold & 3.0 & Curvilinear structure detection \\
\hline Lower threshold & 1.0 & Curvilinear structure detection \\
\hline Linking distance & 5 & Curvilinear structure detection \\
\hline Minimum line length & 5 & Curvilinear structure detection \\
\hline Strength Threshold & 1.0 & Boundary detection \\
\hline MinBackboneLen & 80 & Minimum backbone length \\
\hline
\end{tabular}

\subsection{Conclusion and Discussion}

In this chapter, we have presented a novel approach for automated detection of dendritic backbones and spines in neuron images. The algorithm is able to accurately and smoothly extract the backbones and centerlines of the spines based on the curvilinear structure detection method. A novel boundary detection method is derived and applied to rapidly segment the spine areas. The algorithm can detect and quantitate both attached spines and detached spines. For attached spines, a classifier is used to further remove protrusions based 
on their geometry and moment features. The algorithm is highly automated, requiring minimum or no human interaction. The automated process is fast. The running time is only in minutes for processing a $512 * 512 * 10$ neuron image with a Pentium IV $2.8 \mathrm{G} \mathrm{Hz}$ processor. The experimental results show that the algorithm is able to extract the dendritic backbones and spines accurately and completely, and provide detailed information consistently. Our algorithm outperforms other methods in that it provides fast and fully automated processing and more accurate detection of dendritic backbone and spines in images with poor quality and complicated structures. 

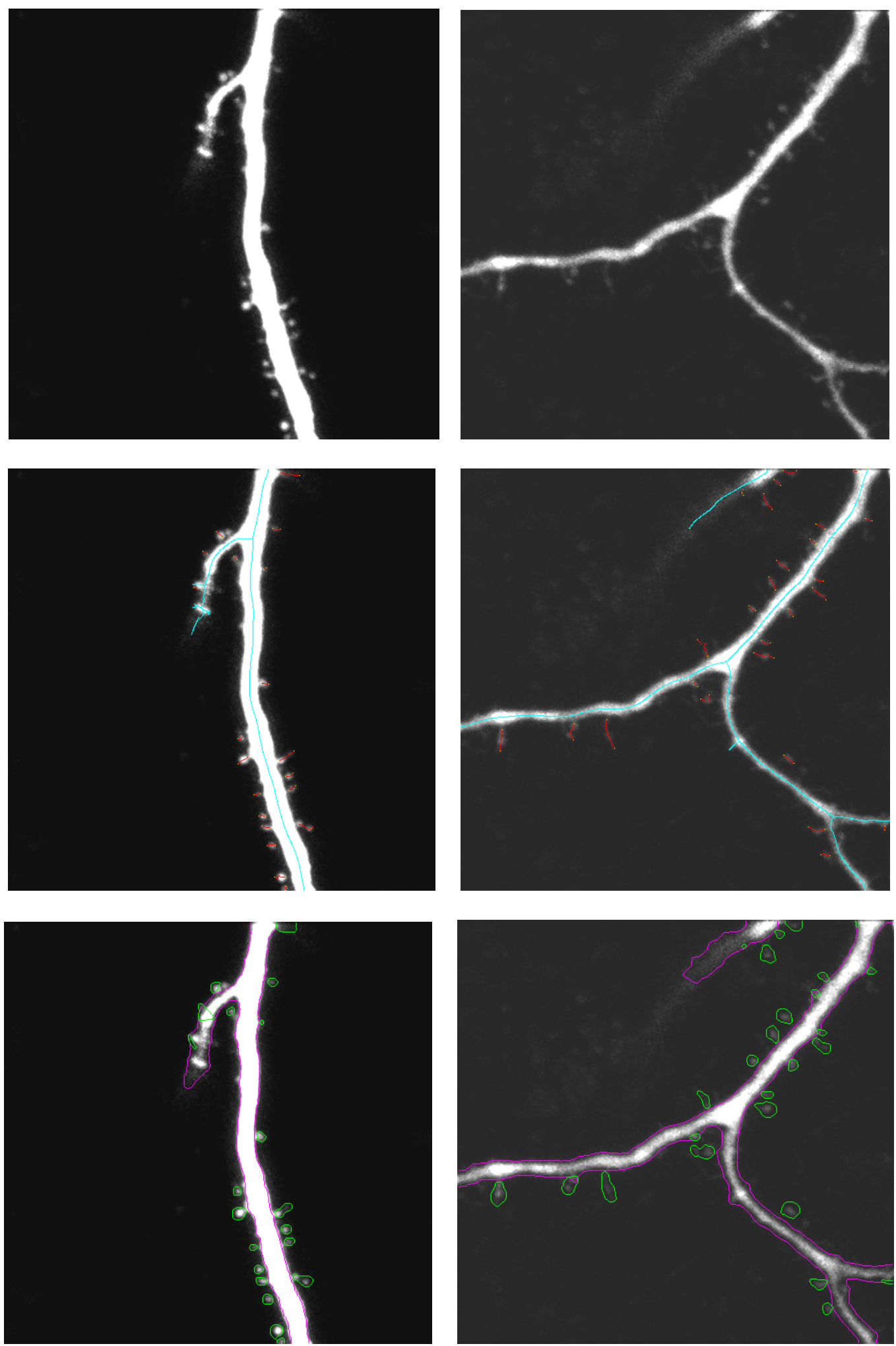

Figure 26. Results for dendritic backbone and spine extraction. The images on the first row are the original neuron images, images on the second row are the results with extracted centerlines labeled with color (cyan for backbone centerlines and red for spine centerlines), and images on the third row are the results with extracted boundaries labeled with color (magenta for backbone boundaries and green for spine boundaries). 

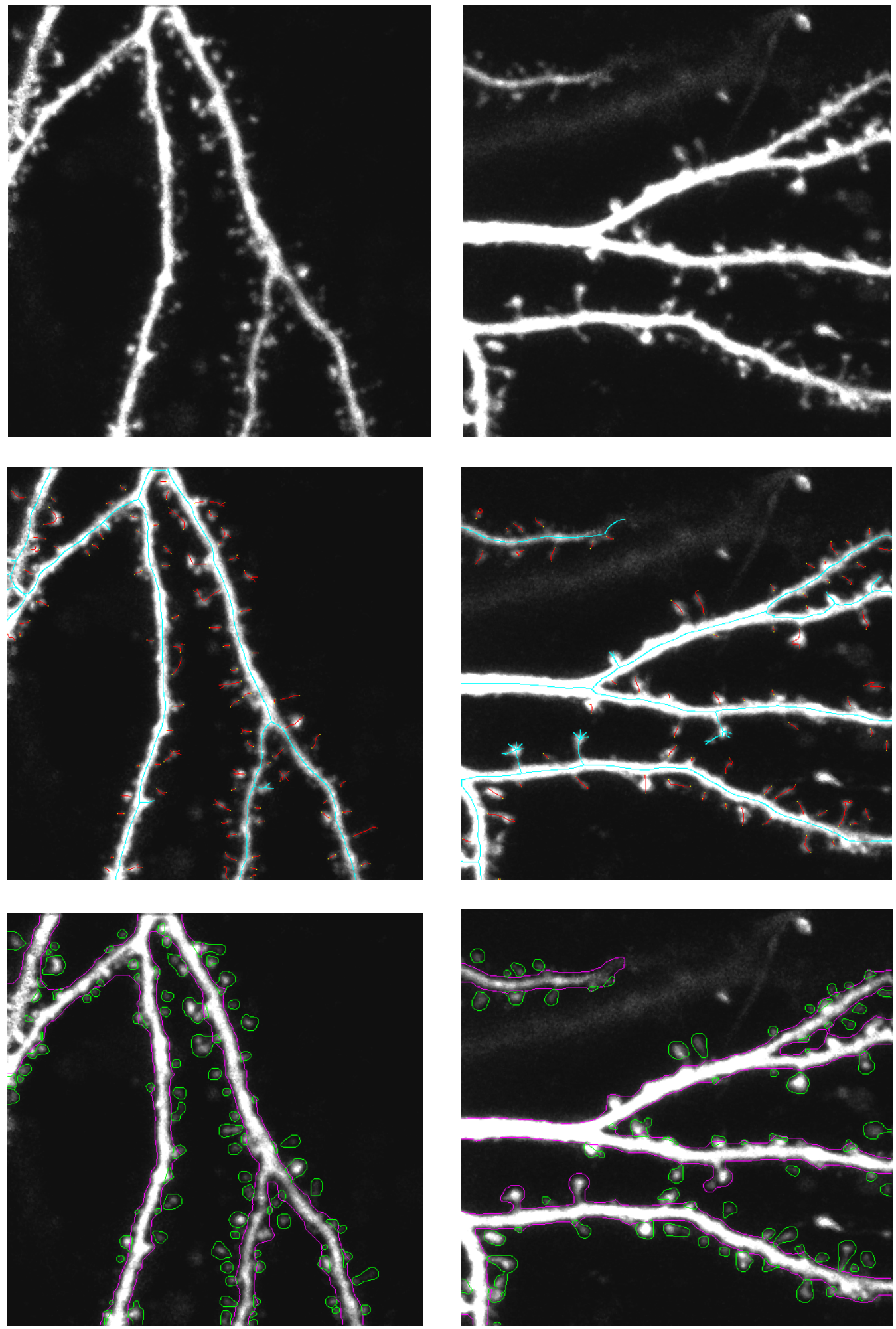

Figure 27. Results for dendritic backbone and spine extraction. The images on the first row are the original neuron images, images on the second row are the results with extracted centerlines labeled with color (cyan for backbone centerlines and red for spine centerlines), and images on the third row are the results with extracted boundaries labeled with color (magenta for backbone boundaries and green for spine boundaries). 


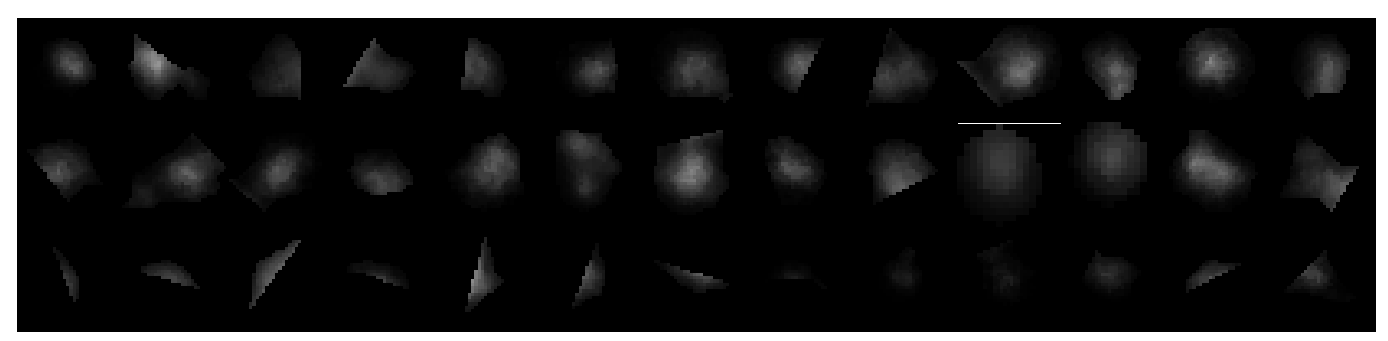

Figure 28. Partial spine samples in the training set for spine classifier. The first two rows are the samples of valid spine type; the last row is the samples of invalid spine type.

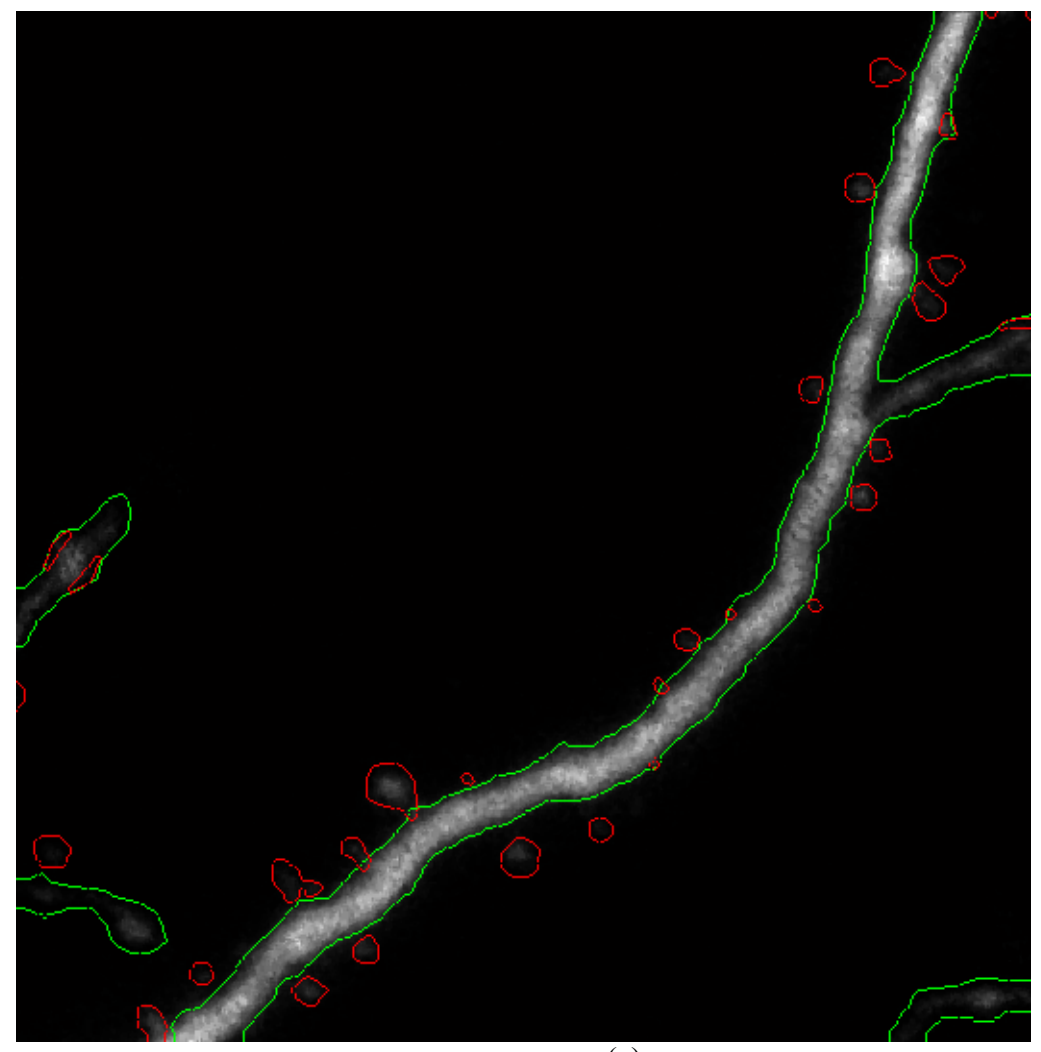

(a) 


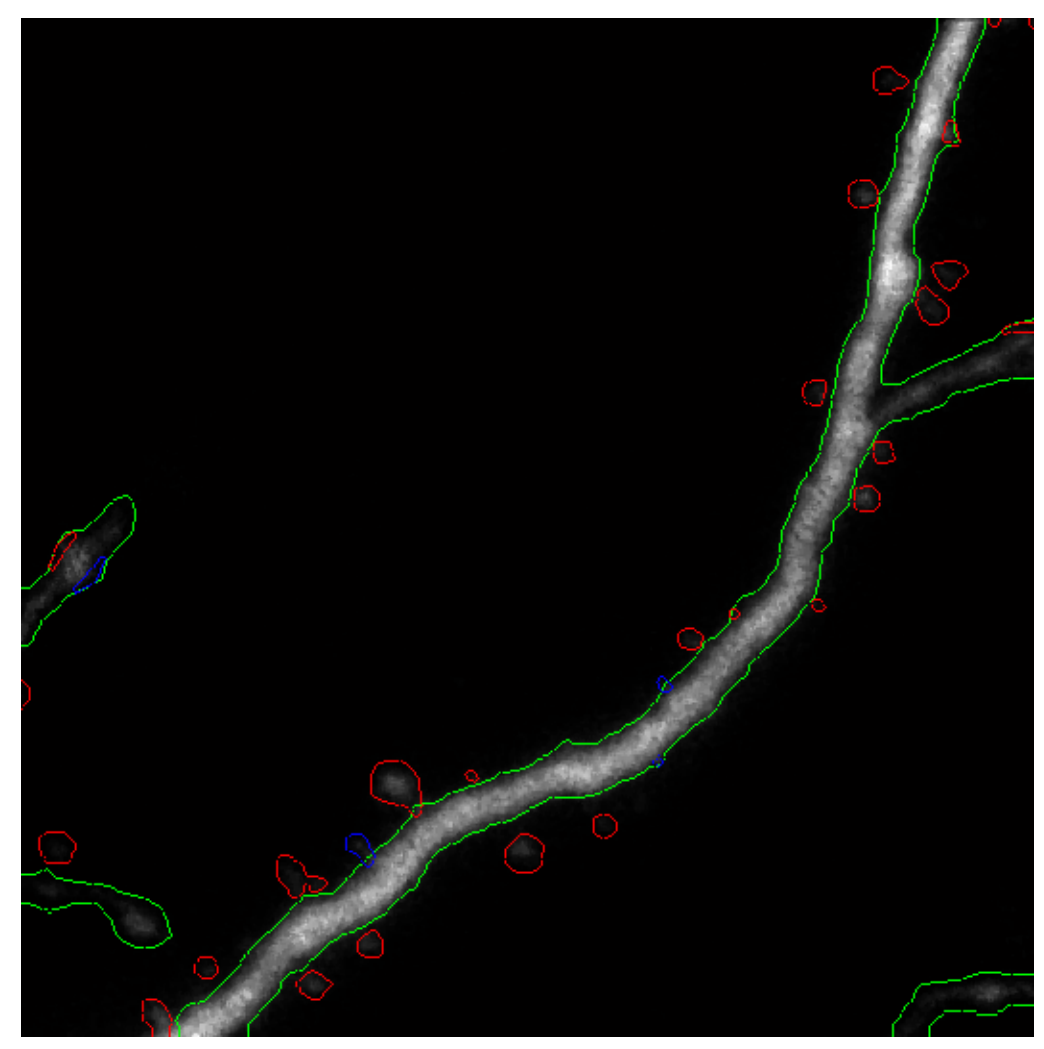

(b)

Figure 29. Spine detection results (a) without spine classification; (b) with spine classification. The dendrite boundaries are labeled in green color; The spines are circled in red color; The pseudo attached spines detected by the classifier are labeled in blue color.

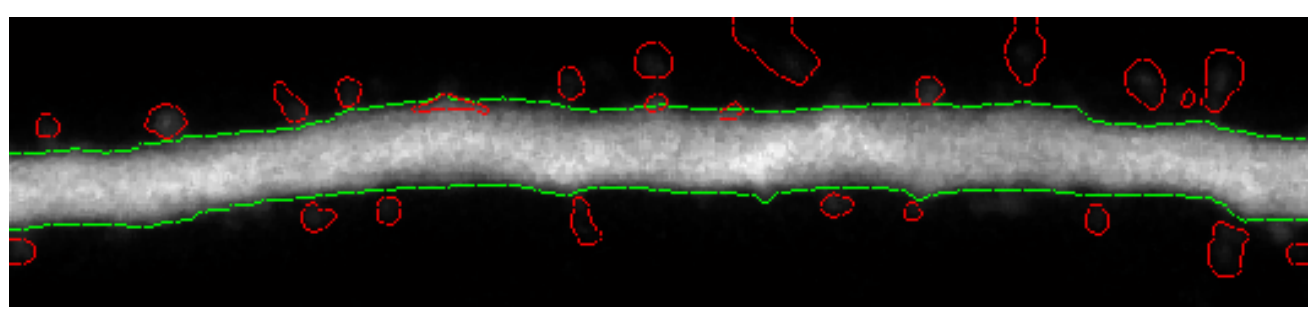

(a)

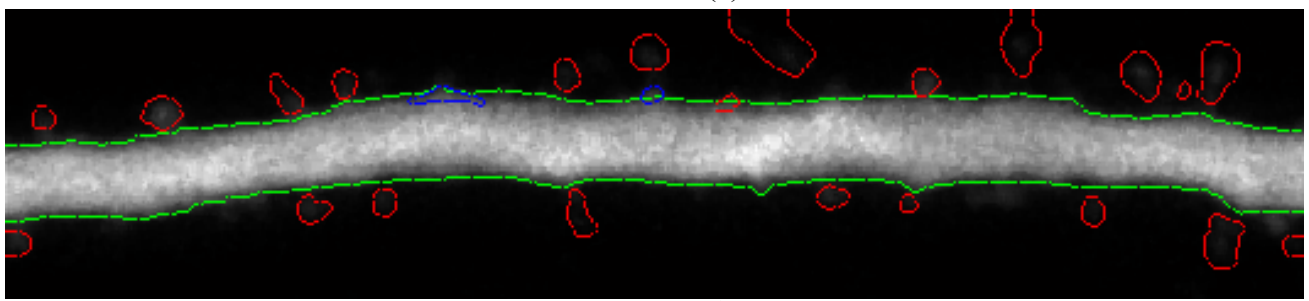

(b) 


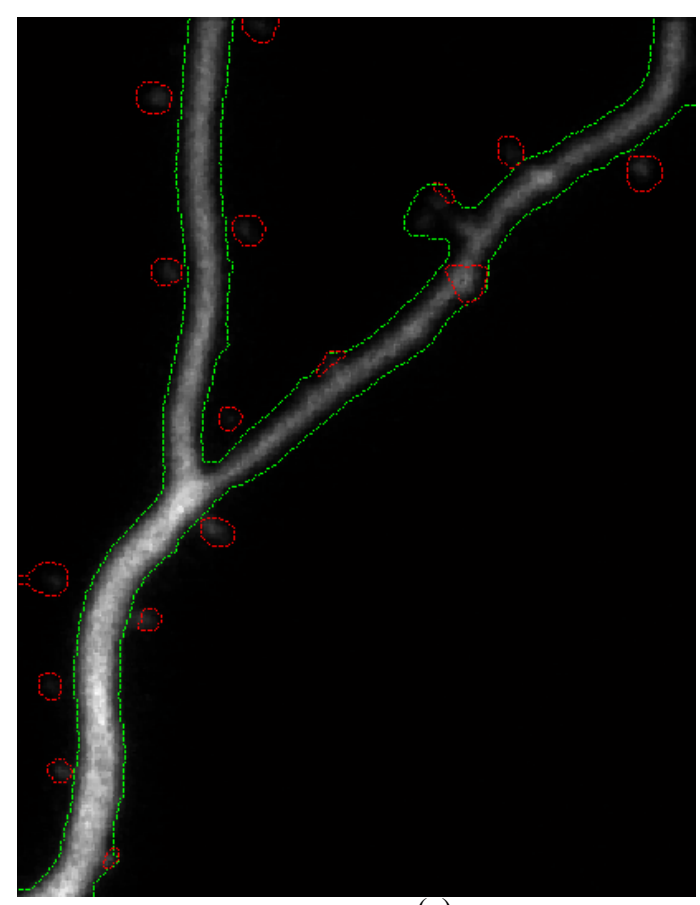

(c)

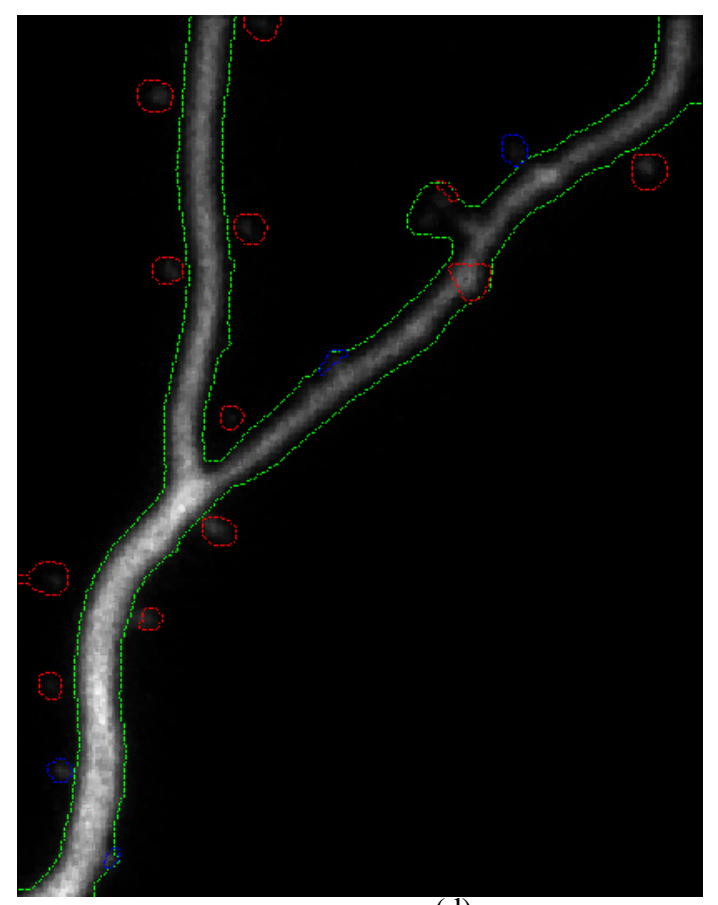

(d)

Figure 30. Spine detection results (a) without spine classification; (b) with spine classification. (c) without spine classification; (d) with spine classification.The dendrite boundaries are labeled in green color; The spines are circled in red color; The pseudo attached spines detected by the classifier are labeled in blue color. 

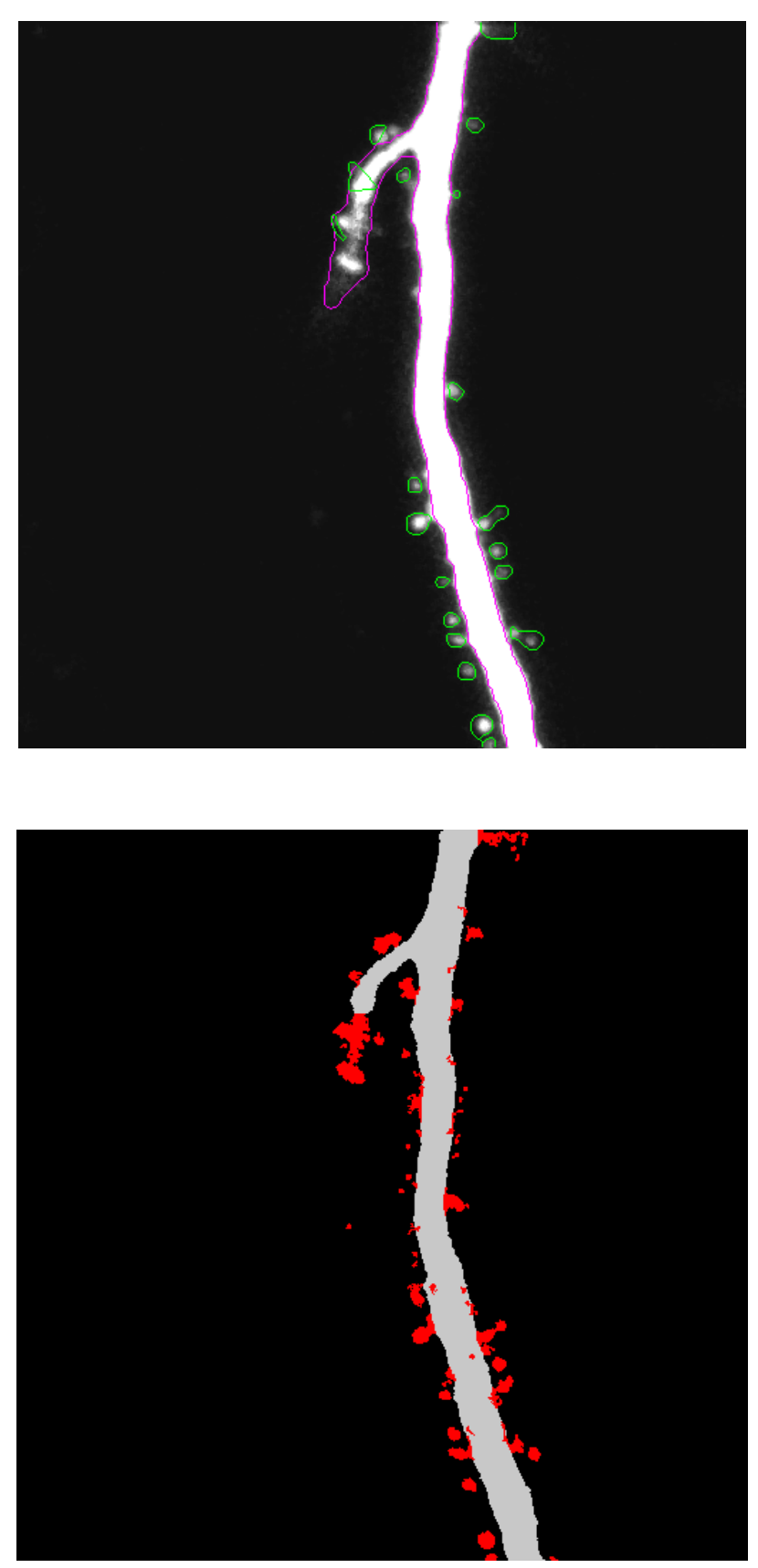

(a) 

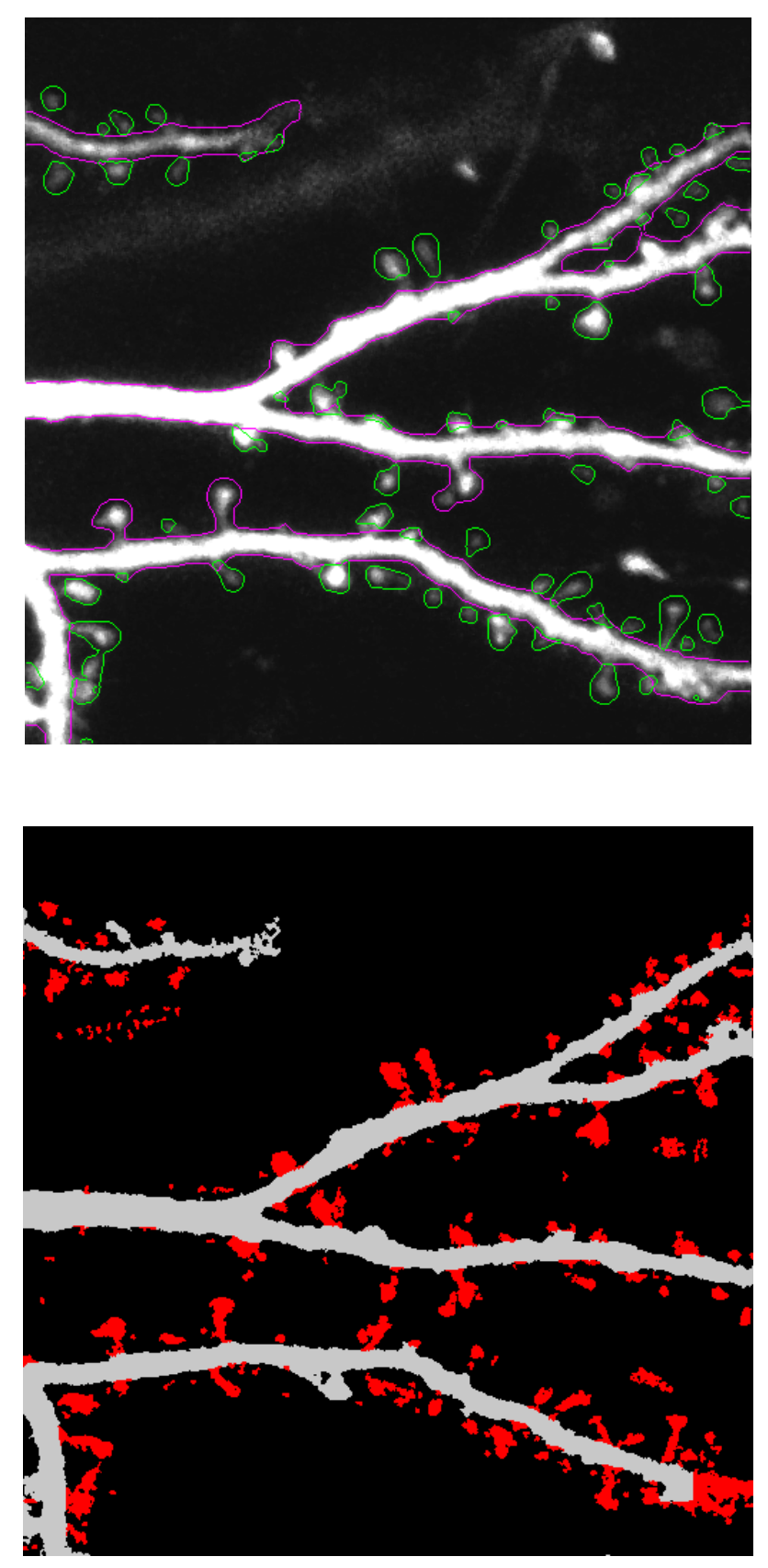

(b)

Figure 31. (a)(b) Two Comparison results between our algorithm and the medial axis and global threshold based algorithm (see [10]). For each row of the two images, the image on the left represents the result produced by our algorithm, the detected spines are labeled by green color; the image on the right represents the result by the previous algorithm, the detected spines are labeled by red color. 

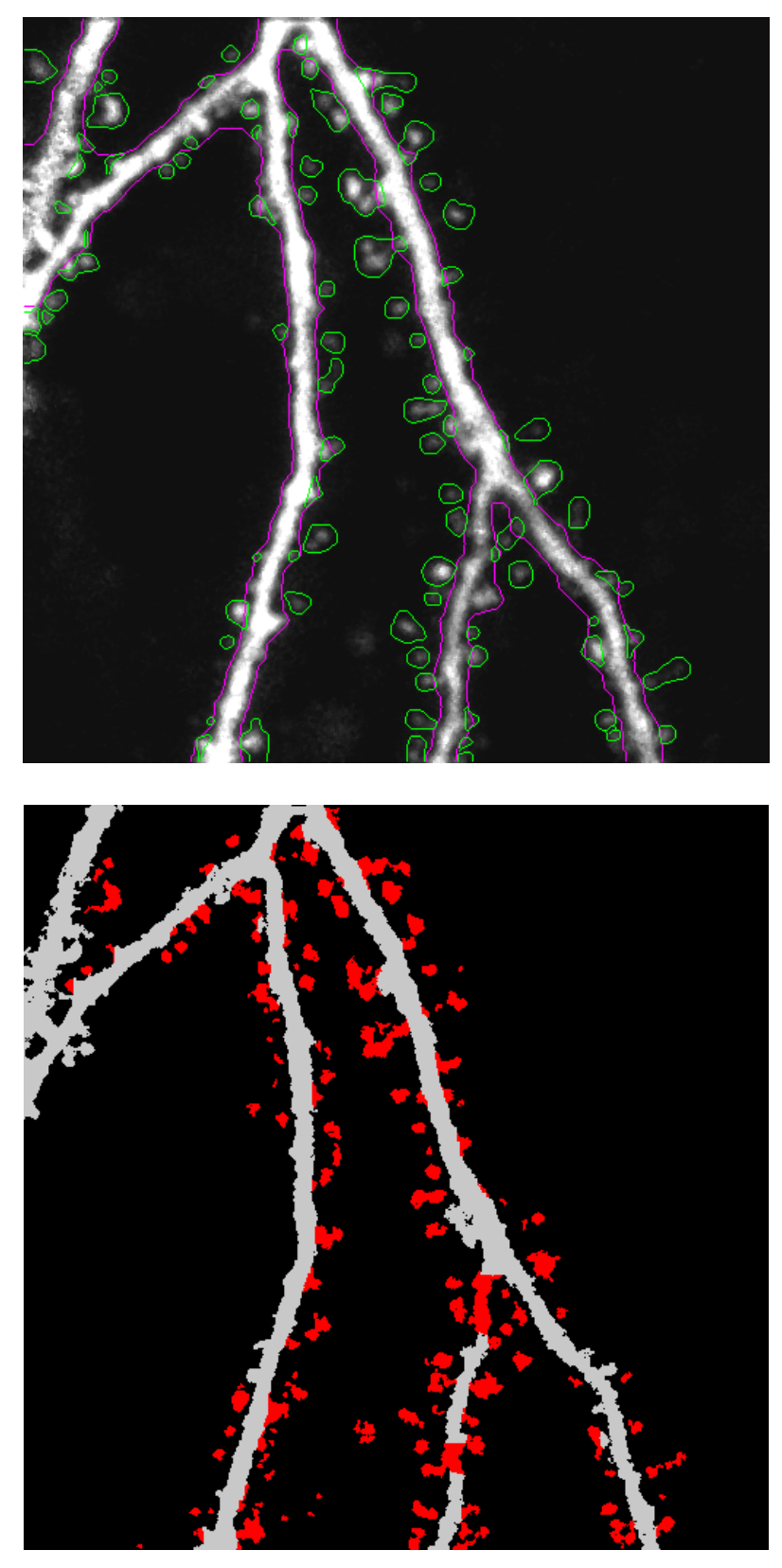

(a) 

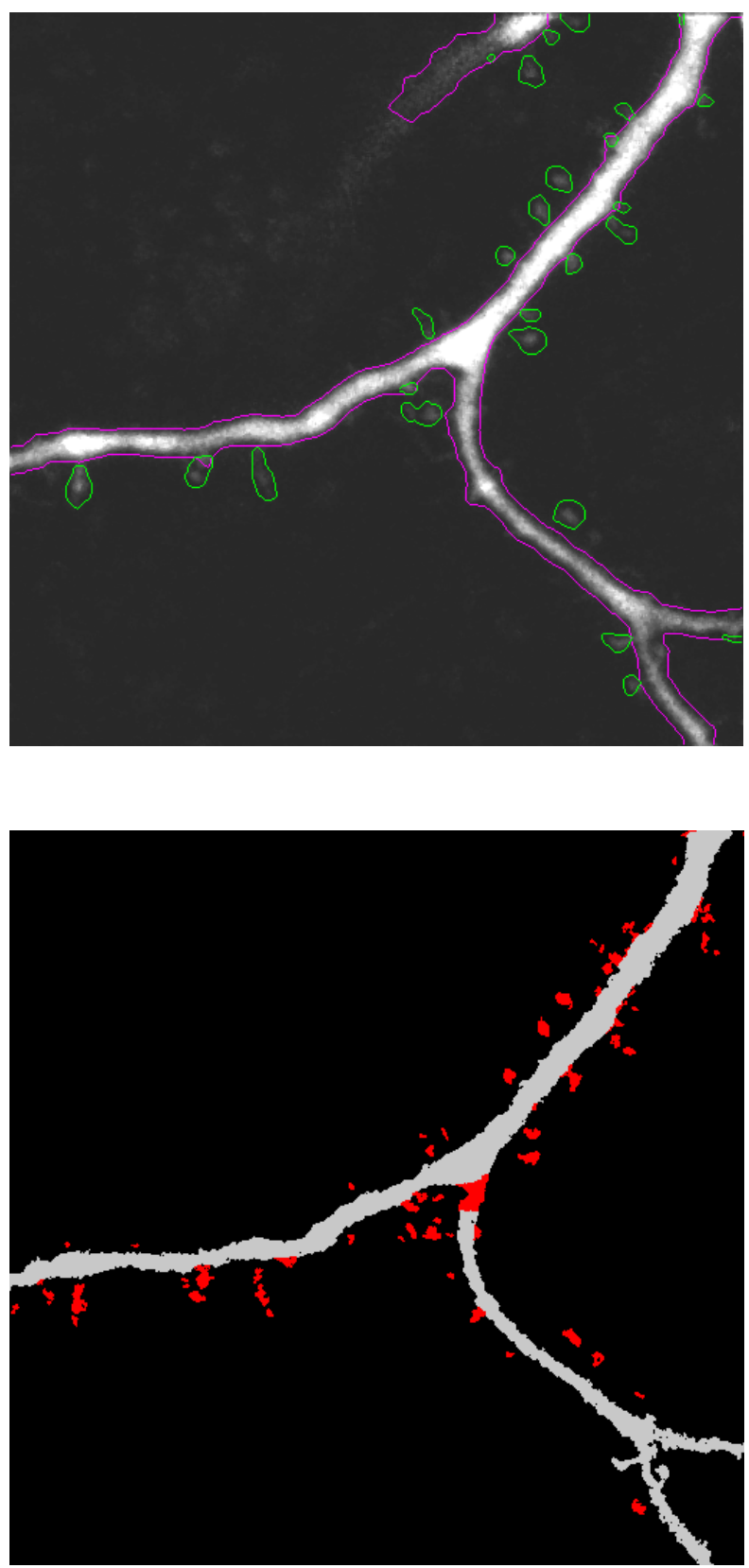

(b)

Figure 32. (a)(b) Two Comparison results between our algorithm and the medial axis and global threshold based algorithm (see [10]). For each row of the two images, the image on the left represents the result produced by our algorithm, the detected spines are labeled by green color; the image on the right represents the result by the previous algorithm, the detected spines are labeled by red color. 


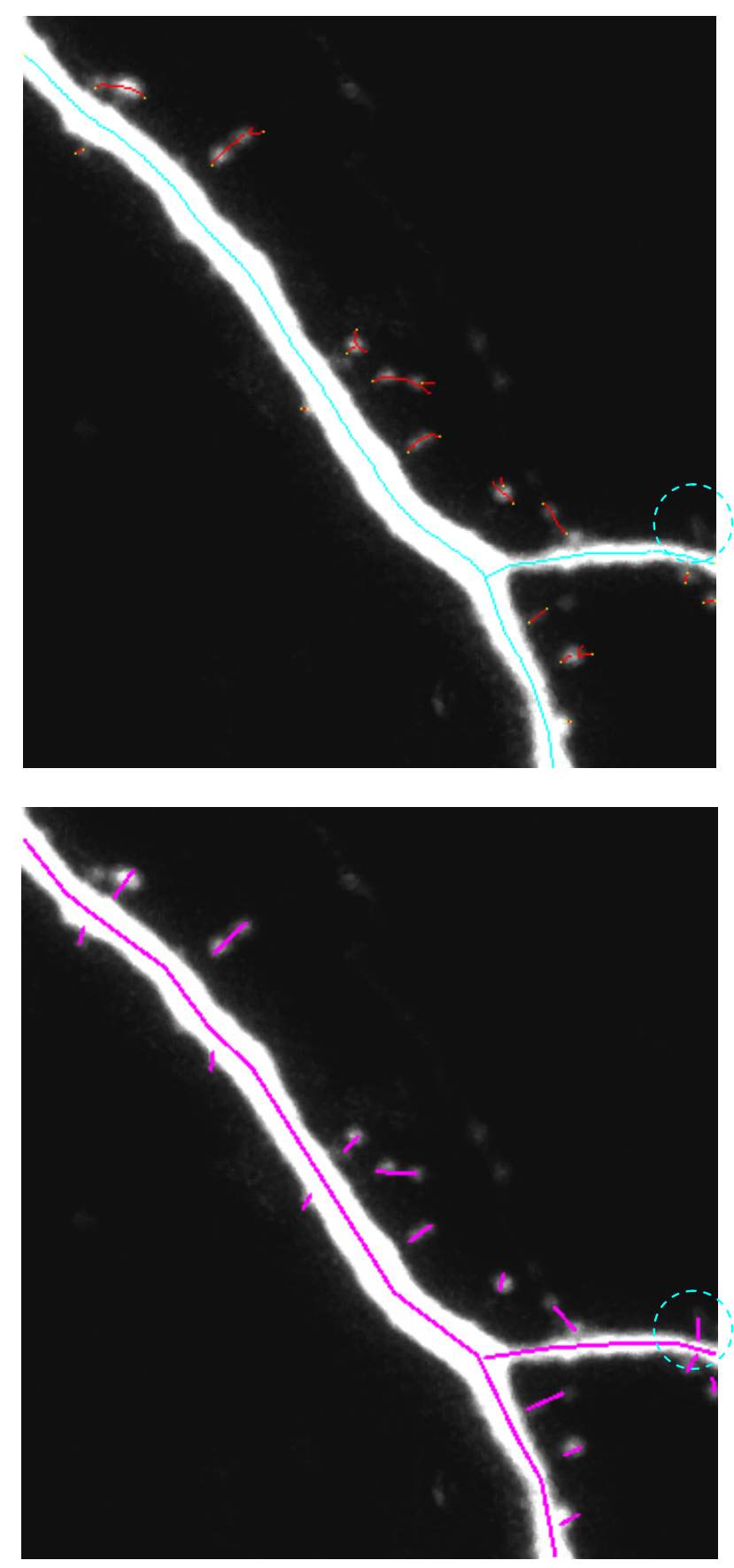



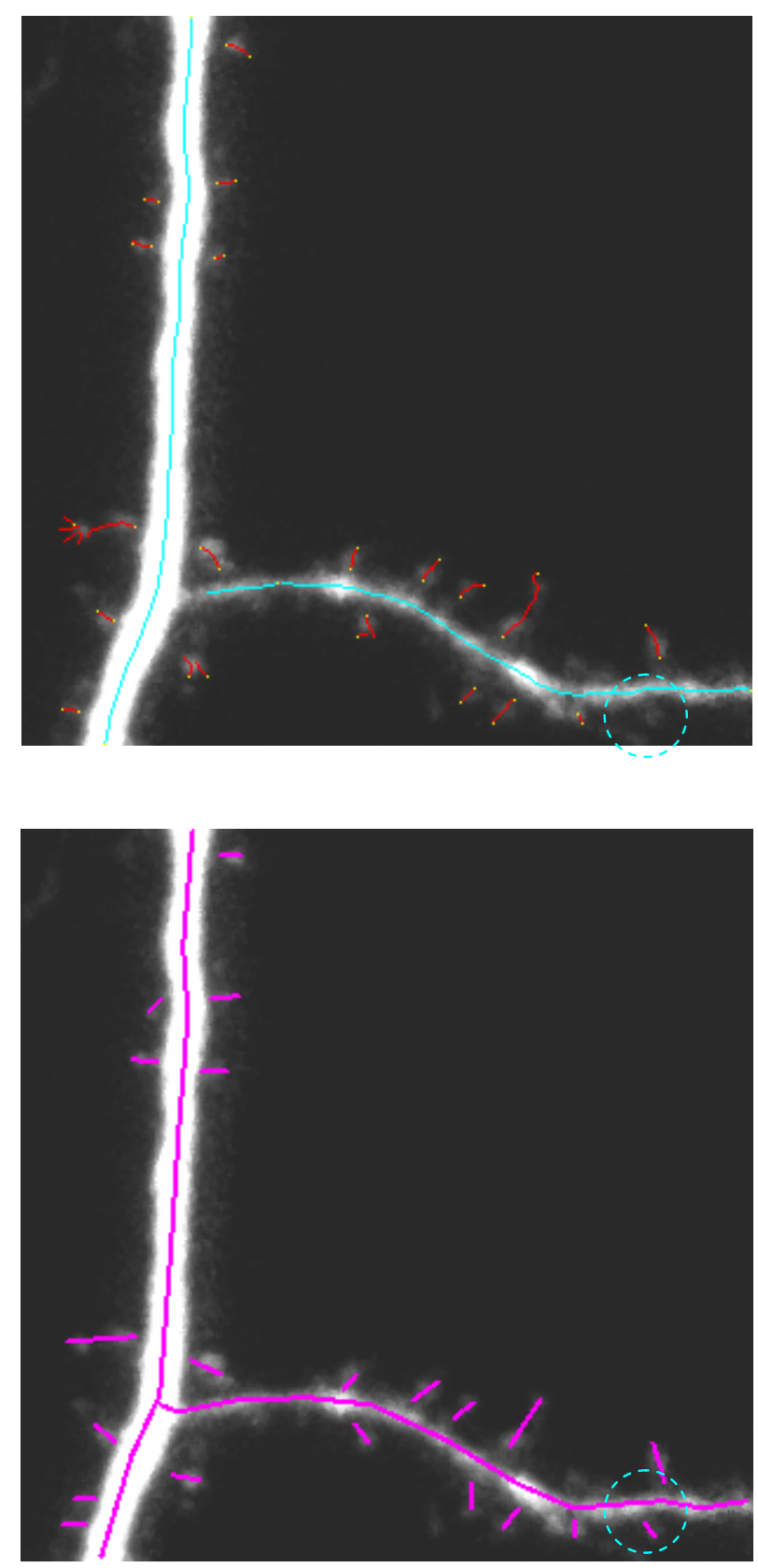

Figure 33. Visual comparison: The two images on the left column are the results from the proposed automated method. The two other images are the results obtained manually. The spines circled in the manual results illustrate the ability of the automated method to remove the pseudo spines due to noise or protrusions. 

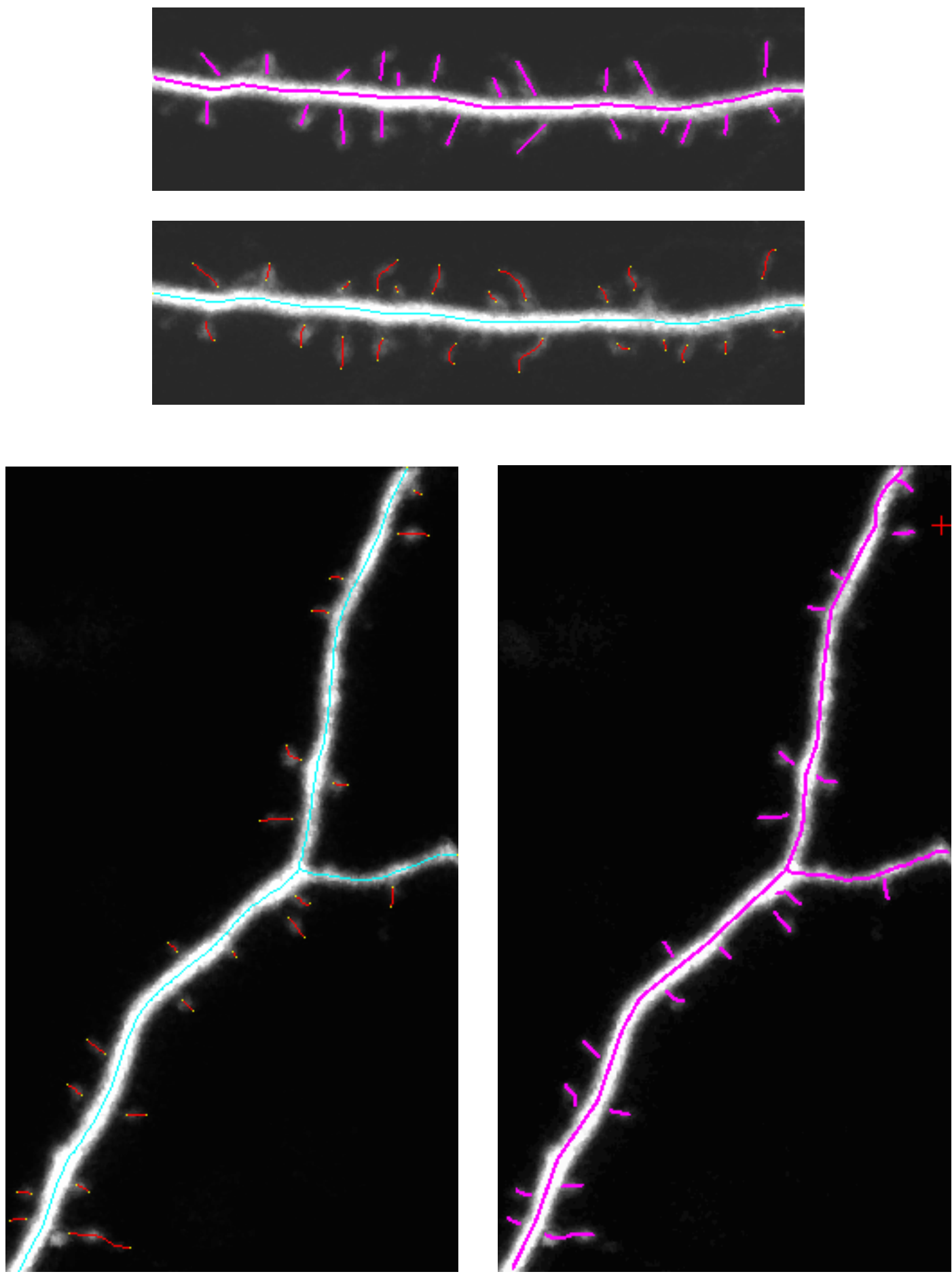

Figure 34. Visual comparison: Two example images on which both automated and manual method obtain the same result. 


\section{Chapter 5: 3D Neurite Centerline Extraction}

Related papers by Yong Zhang for this Chapter:

(1) Y. Zhang, X. Zhou, J. Lu, J. Lichtman, and S. T. C. Wong, "3D curvilinear structure extraction and analysis in fluorescence microscopy images," IEEE Transactions on Biomedical Engineering, under review, 2006. 


\subsection{Introduction}

The morphological properties of neurons, such as their branching patterns and oriented structures, are of great interests for biologists to study the synaptic connectivity of neurons. As an example, the orientation of motor neurons is critical in answering the question regarding synapse elimination in a developing muscle [100]. At neuro-muscular junctions of developing mammals, the neuronal branches of several motor neurons compete with each other which may result in withdrawal of all branches but one [101]. The 3D image reconstruction technique has been widely used to visualize the geometrical features and topological characteristics of the 3D tubular biological objects to help understanding how the morphological properties of the neurons change during the development of the neuronal system.

Digital fluorescent microscopy technique offers tremendous values to localize, identify and characterize cells and molecules in brain slides and other tissues as well as live animals. Accordingly, microscopy imaging and image analysis would be a powerful combination that allows scientists and researchers to gather objective, quantitative and reproducible information, thereby obtaining stronger statistical evidence faster and with fewer experiments. Today the bottleneck in realizing this workflow is the fully automated analysis of large volume of neuron images. However, to design a fully automated image analysis algorithm still remains a very difficult problem, due to the limitation of the computing techniques and the complexity of the 3-D neuronal images. The Vidisector [102, 103] and the UNC automatic neuron-tracing system [104] represent early research work on 3-D neurite tracing problem. The methods have never been widely used due to their poor tracing performance and frustrating use. Cohen et al. [105] proposed a tracing algorithm from 3-D confocal fluorescence microscopy images using segmentation and skeleton extraction algorithm. He et al. [106] extended the work of Cohen using an improved skeletonization algorithm. They 
combined automatic method with semiautomatic and manual tracing in an interactive way in case the dendritic fragments were missed by the automatic process. Al-Kofahi et al. [43] and Lin et al. [107] proposed methods that are superior in terms of speed and accuracy to the work of $\mathrm{He}$ and Cohen for tracing the neurite structures by automatic seed point detection and adaptive tracing templates of variable sizes. Other 3-D neuron structure analysis algorithms are based on vectorization [1, 108-110]. This method models the dendritic structure as cylinders and the tracing is conducted along these cylinders.

Many available software tools, such as NeuronJ [3], Reconstruct [111], and Imaris [112], can extract the centerlines of neuron segments in a semi-automatic way. For instance, NeuronJ can extract very accurately the centerlines of the line structures in the z-projected 2D image. However, it requires that users manually select the starting and ending points. Reconstruct, the software tool which can extract the centerlines from a 3D image stack, also requires that users manually segment different objects if they are attached or overlapped. Imaris, a commercial software package working interactively with the users, still requires that users estimate the orientation and branching patterns of the 3D objects. All of these software tools require intensive manual segmentation of a huge amount of $3 \mathrm{D}$ image data, which is a painstaking task and can lead to fatigue related bias.

A fully automated 3-D extraction and morphometry of tube-like structures usually requires a significant processing time. And the performance is somewhat moderate with the possible presence of sheet-like structures and blob-like shapes in 3-D image stacks. Further, sometimes researchers are only interested in a particular tube-like structure on or near a particular area (for example, in $\mathrm{AD}$ research mentioned at the beginning people only need to monitor the morphological changes of a dendritic structure passing through the A deposits), so a full extraction of all the tube-like structures is not necessary. Based on these facts, we have developed a semi-automated tool to facilitate manual extraction and measurement of 
tube-like neuron structures from 3D image stacks. This tool is somewhat like the 2-D tracing tool proposed by Meijering [3], but now for the analysis of 3D image stack. With this tool, the users only need to interactively select an initial point and then the tube-like structure can be automatically extracted and labelled for further use. There is no need for the users to manually select the ending points. The dendritic geometry such as curvature and length can also be measured automatically to further reduce the tedious manual process. We present in the following sections the design of our proposed approach and the experimental results.

\subsection{Method for 3D Centerline Extraction}

\subsubsection{Test Images}

When studying the mechanism of A deposition disrupting cortical function in Alzheimer's disease (AD), researchers use triple immunofluorescent confocal microscopy to record morphological changes of neuronal processes. Figure 35 shows the projection view (Maximum Intensity Projection or MIP) of one sample 3D neuron image. Three dimensional stacks of images of fluorescently labeled processes are obtained by confocal microscope. Figure 36 shows an example on how the $3 \mathrm{D}$ neuron stack is formed. The left image is a $3 \mathrm{D}$ projection view on the $x-z$ plan. The 3D neuron image stack consists of a series of images that are obtained by imaging the $3 \mathrm{D}$ neuron objects along the $\mathrm{z}$-direction and projecting onto the $\mathrm{x}-\mathrm{y}$ plane. Each slice contains a number of 3D neuron objects projected onto the $\mathrm{x}-\mathrm{y}$ plane, as shown in the figure. The right image shows that the image stack consists of a series of slices. The 3D neuron image contains a number of neurons that are either attached or detached, and may go across others in the 3D space. 


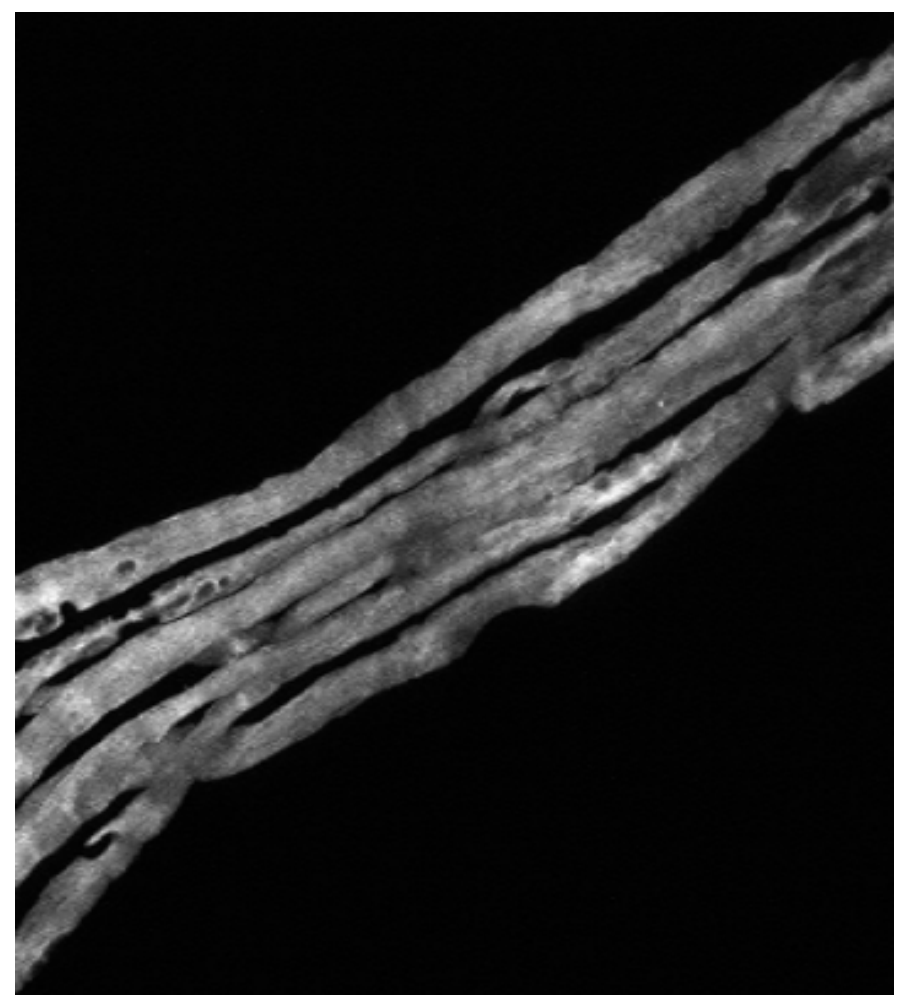

Figure 35. The projection view (Maximum Intensity Projection) of a sample 3D neuron image.
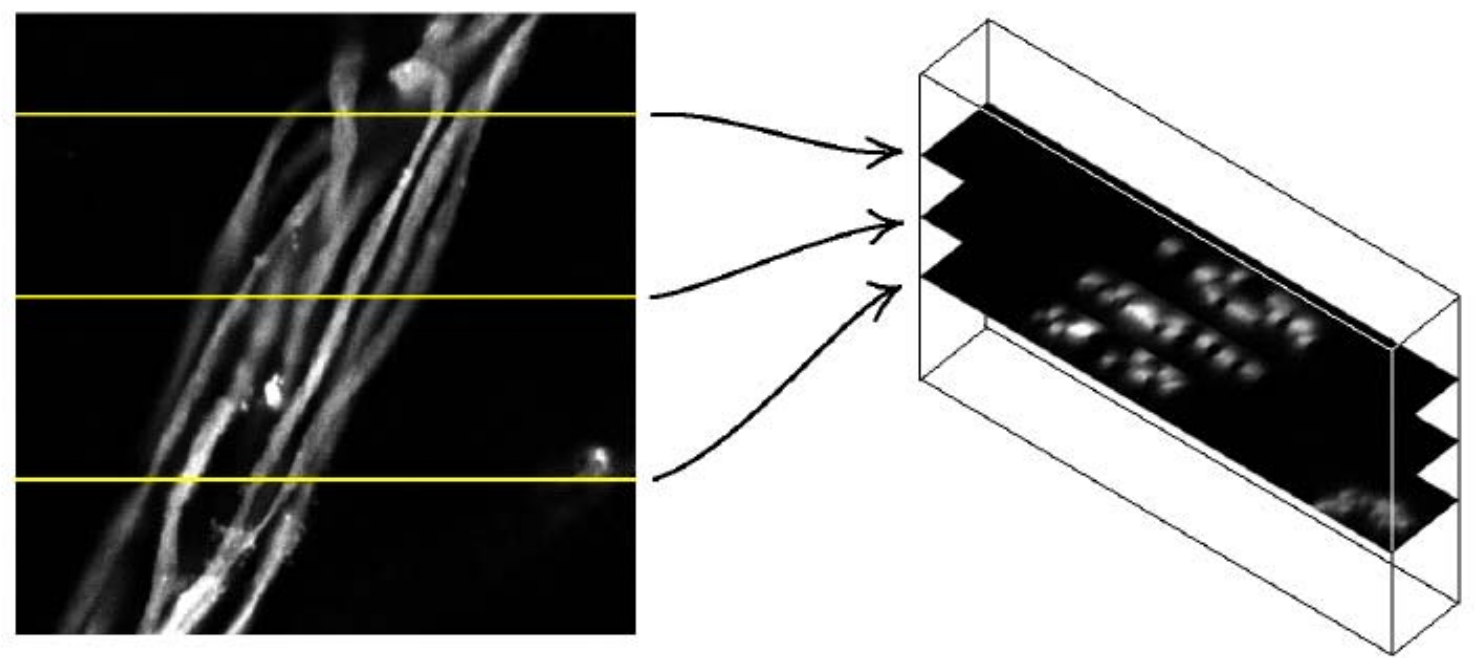

Figure 36. An example on how the 3D neuron image stack is formed. The 3D neuron image stack consists of a series of images that are obtained by imaging the 3D neuron objects along the $\mathrm{z}$-direction and projecting onto the $x-y$ plane. Each slice contains a number of 3D neuron objects projected onto the $x-y$ plane, as shown in the figure. 


\subsubsection{Image preprocessing}

In digital imaging process, noise and artifacts are frequently introduced in the images. So preprocessing is usually a necessary step to remove noise and undesirable features before any further analysis of the images. In this project, a two-step method is used to enhance the image quality and highlight the desired line structures. In the first step, we apply intensity adjustment to all the pixels in the image. An analysis of the histograms from the test images indicate that most images have their 8 -bit intensities falling into the range $[0,127]$. We map the intensity values to fill the entire intensity range $[0,255]$ to improve the image contrast.

In the second step, we apply grayscale mathematical morphological transforms on the neuron images. The morphological transforms are widely used in detecting convex objects in digital images [113]. The top-hat transform of an image $I$ is as follows:

$$
I \rightarrow I_{\text {top }}: I-\max _{R}\left\{\min _{R}\{I\}\right\}
$$

where $I_{\text {top }}$ is top-hat transformed image, $R$ is a structuring element which is chosen to be larger than the potential convex objects in the image I. A disk-shaped flat structuring element with a radius of 3 is used to generate a disk-shaped structuring element, a method called decomposition using periodic lines [114]. Another important morphological transform, bottom-hat transform, can be defined in a similar way. The top-hat transform keeps the convex objects, and the bottom-hat transform contains the gap areas between the objects. The contrast of the objects is maximized by adding together the top-hat transformed image and the original image and then subtracting the bottom-hat transformed image:

$$
J=I+I_{\text {top }}-I_{b o t}
$$

where $I_{b o t}$ is the bottom-hat transformed image. 


\subsubsection{Centerline Extraction in 3D Neuron Images}

We have discussed the Dynamic Programming (DP) technique for 2D centerline extraction in Chapter 4. The dynamic programming is able to extract the centerlines accurately and smoothly if both the starting and ending points are specified. However, given the complexity and dimensionality of a 3D image stack, it is usually difficult for the users to select the correct pair of starting-ending points. Thus, the direct use of the conventional DP technique is not suitable to address the problem of extracting centerlines in 3D neuron image stacks. Another problem with extending the DP technique is that it is very computational expensive to search for all the optimal paths for each point in the 3D image stack.

In order to solve these problems, we combine the DP technique with marker-controlled watershed segmentation and tracking. Our proposed method is based on the following assumptions: (1) there is only one object for each neuron on each slice, i.e., each neuron goes through each slice only once; (2) the neurons change directions smoothly along their navigations. These assumptions can be easily observed from many sample 3D neuron images (we consider it as our future work if the 3D neuron images have more complicated structures that do not meet the above assumptions).

Our proposed method can be briefly described as follows: the extraction starts from a set of pre-defined seed points on the first slice of the 3D image stack; from each seed point, instead of searching for all the optimal paths for the entire 3D stack, we dynamically search for optimal paths for the points that are within a small region (a $10 \times 10$ searching region centered at the seed point is enough to cover the possible neuron object in the following slice) on the following slice; A cost value is calculated for each point based on its optimal path; the point with the minimum cost value is used as a marker and the watershed method is applied based on all the markers to segment the slice into $2 \mathrm{D}$ neuron objects; the centroids of the segmented regions are used as the detected centerline points and new searching is applied 
starting from each of these points, until the last slice. We describe the details in the following subsections.

\subsubsection{Optimal Path Searching and Cost Value Calculation}

In conventional DP method, the optimal path searching involves all the neighborhoods of the current point. In this project, we have assumed that the neurons change their directions smoothly and enter each slice only one time. Thus we can ignore the previous slice and examine only the points on the following slice. We further reduce the computational complexity by considering only a set of neighboring points within a small region centered at the seed point. We call this region the Search Region and denote it with $S$. In this work, the small region has a size of $10 \times 10$. In general, if there are $k$ seed points (that means $k$ neurons), then there will be $k$ such small regions and the search region consists of $k$ regions:

$S=\bigcup_{i=1}^{k} s_{i}$, where $s_{i}, i=1, \cdots k$ represents the $i-$ th small $10 \times 10$ region.

The optimal path searching consists of two steps. In the first step, every point in the image stack is assigned a similarity measurement to the $3 \mathrm{D}$ line structure using $3 \mathrm{D}$ line filtering. Previous research in modeling multi-dimensional line structure is exemplified by the work of Steger et al. [51] and Sato et al. [50]. In this work, the 3D line filtering is achieved by Hessian matrix (ref), a descriptor that is widely used to describe the second-order structures of local intensity variations around each point in the 3D image stack. The Hessian matrix for a pixel $p$ at location $(x, y, z)$ in a 3-D image stack $I$ is defined as:

$$
\nabla^{2} I(p)=\left(\begin{array}{ccc}
I_{x x}(p) & I_{x y}(p) & I_{x z}(p) \\
I_{y x}(p) & I_{y y}(p) & I_{y z}(p) \\
I_{z x}(p) & I_{z y}(p) & I_{z z}(p)
\end{array}\right)
$$


where $I_{x x}(p)$ represents the partial second order derivatives of the image at point $p$ along the $x$ direction, and $I_{y z}(p)$ is the partial second order derivatives of the image along the $y$ and $z$ directions, and so on. The eigenvalues of $\nabla^{2} I(p)$ are used to define the similarity measurement of a point to a 3D line structure. If we denote the three eigenvalues as $\lambda_{1}, \lambda_{2}, \lambda_{3}$ in descending order, (that is, $\lambda_{1}>\lambda_{2}>\lambda_{3}$ ), the similarity measure $\eta(p)$ for point $p$ is calculated as follows:

$$
\eta(p)= \begin{cases}\left|\lambda_{2}\right|+\lambda_{1}, & \text { if } \lambda_{1} \leq 0 \\ \left|\lambda_{2}\right|-\frac{\lambda_{1}}{4}, & \text { if } 0<\lambda_{1}<4\left|\lambda_{2}\right| \\ 0 & \text { otherwise }\end{cases}
$$

Notice that the value of $\eta(p)$ is always greater than or equal to zero. By defining this measure the algorithm can distinguish 3D line structures from sheet-like structures and bloblike shapes. The higher the value of $\eta(p)$, the more likely that the point is belonging to a 3D line structure. The highest value of $\eta(p)$ is expected to indicate a point that is located near or on the centerline, a filled tube-like structure commonly seen in our 3D neuron images. We further convert $\eta(p)$ to a cost value $c(p)$ for point $p$ as follows:

$$
c(p)=1-\frac{\eta(p)}{\max _{p \in I}\{\eta(p)\}}
$$

the value of $c(p)$ decreases if the point goes near the centerline of the 3-D line structure, and it is minimized on the centerline.

In the second step, we search for an optimal path for each point on the current and following two slices to the seed point. The searching method is similar to the DP technique for $2 \mathrm{D}$ centerline extraction, as described in Section 4.1. The only difference is that the searching is applied on the 3D space. Only two slices at a time are involved in the optimal 
path searching. Thus the processing time can be significantly reduced. The cost of linking from point $p$ to point $q$ in the 3D space is determined by the gradient directions of the two points. Let $\overrightarrow{d_{p}}$ denote the unit vector of the gradient direction at $p$ and $\overrightarrow{n_{p}}$ the unit vector perpendicular to $\overrightarrow{d_{p}}$, then:

$$
\begin{aligned}
& \overrightarrow{d_{p}}=\left[I_{x}(p), I_{y}(p), I_{z}(p)\right] \\
& \overrightarrow{n_{p}}= \begin{cases}{\left[\frac{I_{x}(p) I_{z}(p)}{I_{x y}(p)}, \frac{I_{y}(p) I_{z}(p)}{I_{x y}(p)},-I_{x y}(p)\right],} & \text { if } I_{x}(p) \neq 0 \text { or } I_{y}(p) \neq 0 \\
{\left[I_{z}(p), I_{y}(p), I_{x}(p)\right],} & \text { if } I_{x}(p)=0 \text { and } I_{y}(p)=0\end{cases}
\end{aligned}
$$

where $I_{x}(p), I_{y}(p), I_{z}(p)$ represent the unit vectors along $x, y, z$ directions of $p$, respectively. $\overrightarrow{n_{p}}$ here is obtained by rotating $\overrightarrow{d_{p}} 90^{\circ}$ counterclockwise on the xy-z plane and dividing the $I_{z}(p)$ into two components along the $\mathrm{x}$ and $\mathrm{y}$ axis, respectively. $I_{x y}(p)=\sqrt{I_{x}^{2}(p)+I_{y}^{2}(p)}$. The linking cost between $p$ and $q$ is:

$$
d(p, q)=\frac{2}{3 \pi}\left[\cos ^{-1}\left(\overrightarrow{n_{p}} \cdot \overrightarrow{v_{p q}}\right)+\cos ^{-1}\left(\overrightarrow{v_{p q}} \cdot \overrightarrow{n_{q}}\right)\right]
$$

where $\overrightarrow{v_{p q}}$ is the normalized bidirectional link between $p$ and $q$ :

$$
\overrightarrow{v_{p q}}=\left\{\begin{array}{l}
\frac{\vec{q}-\vec{p}}{\|\vec{p}-\vec{q}\|} \text {; if } \overrightarrow{n_{p}} \cdot(\vec{q}-\vec{p}) \geq 0 \\
\frac{\vec{p}-\vec{q}}{\|\vec{p}-\vec{q}\|} \text {; if } \overrightarrow{n_{p}} \cdot(\vec{q}-\vec{p})<0
\end{array}\right.
$$

where $\vec{p}=\left[p_{x}, p_{y}, p_{z}\right]$ and $\vec{q}=\left[q_{x}, q_{y}, q_{z}\right]$ are the vectors of the two points $p, q$ represented by their coordinates. $\overrightarrow{v_{p q}}$ is calculated such that the difference between $\vec{p}$ and the linking direction is minimized. The linking cost between $p$ and $q$, if calculated by formula (67), yields a high value if the two points have significantly different gradient directions or the bidirectional link between them is significantly different from either of the two gradient 
directions. The coefficient $\frac{2}{3 \pi}$ is used to normalize the linking cost $d(p, q)$. It is obtained from the fact that: (1) the minimum possible angle between $\overrightarrow{v_{p q}}$ and $\overrightarrow{n_{p}}$ is $0^{\circ}$ such that $\overrightarrow{n_{p}} \cdot \overrightarrow{v_{p q}} \rightarrow 0$ and $\cos ^{-1}\left(\overrightarrow{n_{p}} \cdot \overrightarrow{v_{p q}}\right) \rightarrow \frac{\pi}{2} ;(2)$ the maximum possible angle between $\overrightarrow{v_{p q}}$ and $\overrightarrow{n_{q}}$ is $180^{\circ}$ such that $\overrightarrow{v_{p q}} \cdot \overrightarrow{n_{q}} \rightarrow-1$ and $\cos ^{-1}\left(\overrightarrow{v_{p q}} \cdot \overrightarrow{n_{q}}\right) \rightarrow \pi$. Thus, the maximum possible value of $\cos ^{-1}\left(\overrightarrow{n_{p}} \cdot \overrightarrow{v_{p q}}\right)+\cos ^{-1}\left(\overrightarrow{v_{p q}} \cdot \overrightarrow{n_{q}}\right)$ is $\frac{3 \pi}{2}$.

The total cost value of linking point $p$ and $q$ can be calculated as follows:

$$
\operatorname{Cost}(p, q)=w_{c}[c(p)+c(q)]+w_{d} d(p, q)
$$

where $c(p)$ is the local cost of $p$ and can be calculated by formula $(65), d(p, q)$ is the linking cost from $p$ to $q$ and is calculated by formula (67). $w_{c}, w_{d}$ are the weights of the local cost and linking cost, respectively. By default, $w_{c}=0.4, w_{d}=0.2$. The marker points (denoted as $\left.m_{i}, i=1, \cdots, k\right)$ are selected as follows:

$$
m_{i}=\underset{p \in S}{\arg \min }\left\{\operatorname{Cost}\left(p, \text { seed }_{i}\right)\right\}, i=1, \cdots, k
$$

That means, the point with the minimum overall cost to the seed point is selected as a marker point. Figure 37 shows an example on how the marker points are selected. The dashed region on the $\mathrm{i}+1$-th slice represents the search region $S$, which consists of two small regions centered at the two seed points projected from the i-th slice. The two points in red and blue colors have the minimum overall cost to the corresponding seed points, thus are selected as the marker points for further segmentation by the watershed method. 


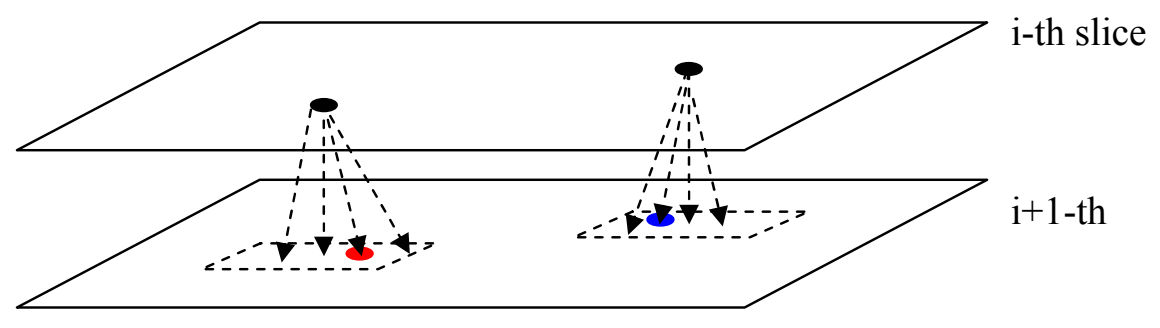

Figure 37. Marker points selection. The two points in colors are the selected marker points on the $i+1$-th slice. The search region for each point is shown as a dotted rectangle.

\subsubsection{Marker-Controlled Watershed Segmentation}

One difficult problem in 3D centerline extraction is that some 3D objects may attach to or go across others in 3D space. If two centerlines are close enough, previous described extraction process may combine two centerlines to be one centerline and the other centerline is totally lost. To solve this problem, we use the marker-controlled watershed to segment different objects on one slice. The centroids of the segmented regions are then used to refine the locations of the centerline points.

The watershed transform has been widely used for image segmentation [115]. It treats a grayscale image as a topographic surface and floods this surface from its minima. If the merging of the water coming from different sources is prevented, the image is then partitioned into different sets: the catchment basins and the watershed lines. However, in practice, this transform may produce over-segmentation due to noise or local irregularities. The watershed transform leads to better segmentation if foreground objects and background locations can be identified as markers and the topographic surface is flooded from these markers. This is called marker-controlled watershed.

Our algorithm for marker-controlled watershed segmentation is as follows:

(A) Calculate the gradient image from the original image slice: the magnitude of the 
image gradient at point $p$ is calculates as follows:

$$
|\nabla I(p)|=\sqrt{\left(\frac{\partial I(p)}{\partial x}\right)^{2}+\left(\frac{\partial I(p)}{\partial y}\right)^{2}}
$$

(B) Calculate the foreground markers: the foreground markers are automatically detected as the minimal cost points using the optimal path searching method described in Section 2.3.1;

(C) Calculate the background markers: First, the original image is segmented into foreground and background using the global threshold [64]; Second, we apply distance transform to the image background. Third, we apply conventional watershed transform to the result of distance transform and keep only the watershed ridge lines as the background markers.

(D) Modify the gradient image so that the regional minima will be at the locations of the foreground and background markers.

(E) Apply marker-controlled watershed transform to the modified gradient image.

(F) Calculate the centroids of the segmented regions and use the results as the refined centerline points.

Figure 38 illustrates the above procedure. Figure 38(a) is the original image slice and (b) gives the segmentation result using conventional watershed. It is obvious that the sample image is over-segmented. Figure 38(c) and (g) are the gradient images before and after the modification using markers. We notice that the regional minima are better defined by the markers in (g) than those in (c). Figure 38(e) shows a total of five foreground markers. These markers are obtained by detecting the five minimal cost points if connected to the five centerline points in the preceding slice. Figure 38 (h) gives the enhanced watershed segmentation result. The five smallest regions (in this example, there are totally five neurites in the 3D neuron image) are considered to adjust the centerlines for the current slice. 


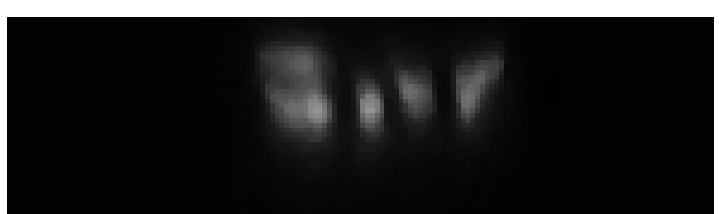

(a)

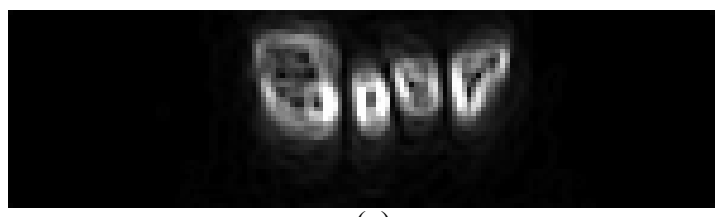

(c)

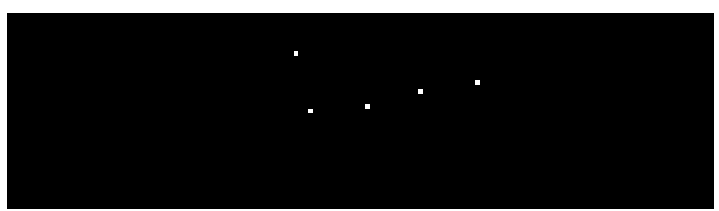

(e)

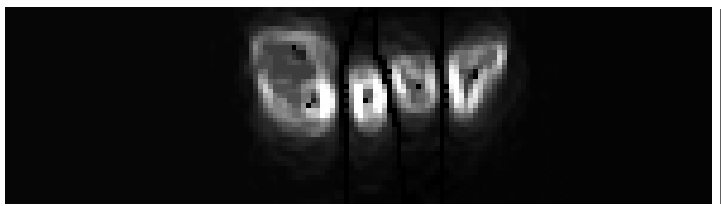

(g)

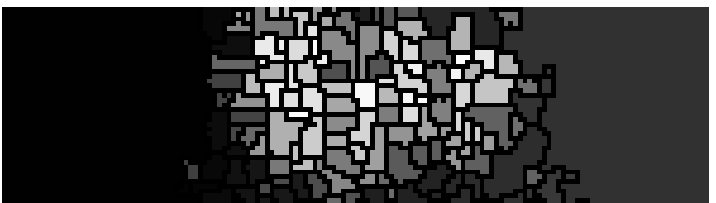

(b)

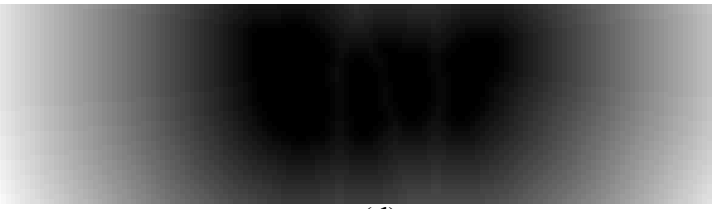

(d)

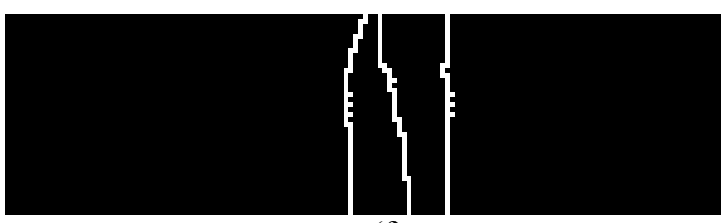

(f)

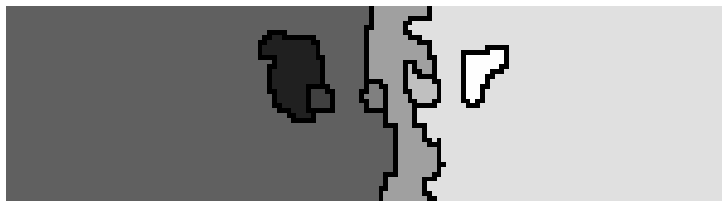

(h)

Figure 38. Example of marker-controlled watershed segmentation. (a) Original image slice; (b) conventional watershed segmentation result; (c) gradient image; (d) distance transform of image background; (e) foreground markers; (f) background markers; (g) modified gradient image; (h) marker-controlled watershed segmentation result.

\subsection{Results}

We apply our 3D centerline extraction algorithm on test image stacks obtained partially from the 3D neuron images as shown in Figure 36. A typical test image stack has a total of 512 slices $(43 \times 512$ for each slice). Figure 39 shows the $3 \mathrm{D}$ view of the surface rendering for the neuron image and the extracted centerlines. It is obvious that the proposed algorithm is able to completely extract the five centerlines for the five neurons in the $3 \mathrm{D}$ space. The extraction is accurate when the neurons are attaching or going across others, as shown in the figure. Figure 40 and Figure 41 show two partial extraction results with and without marker- 
controlled watershed segmentation. It can be seen that the marker-controlled watershed helps to segment different 3D neuron objects if they are attached together or are very close to each other, which otherwise may lead to incorrect extraction of the corresponding centerlines.

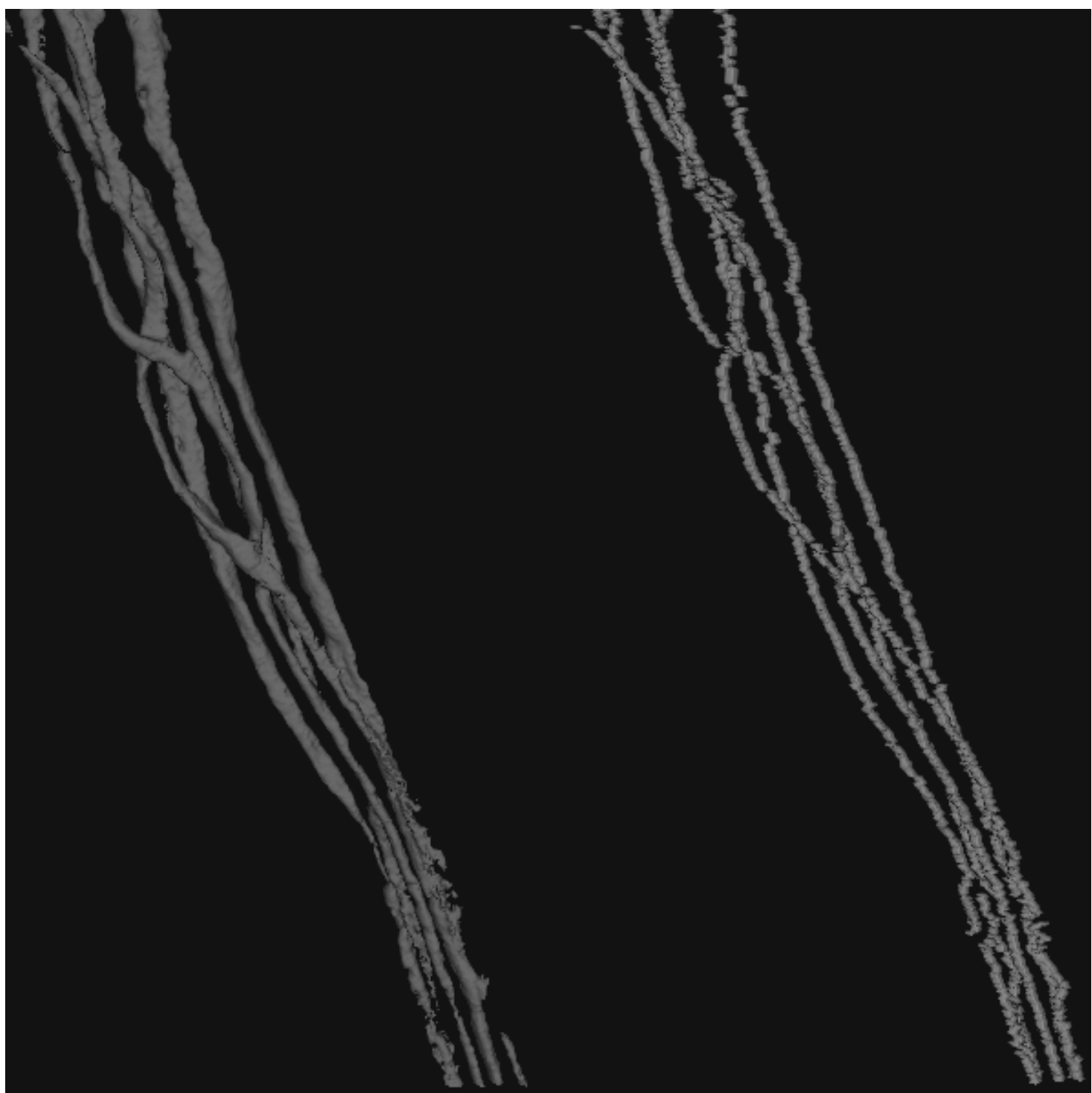

Figure 39. A sample result for 3D centerline extraction. The image on the left is the 3D view of the surface rendering for the original 3D neuron image. The image on the right is the extracted centerlines. The proposed algorithm is able to accurately extract all the centerlines for the five neurons in the image stack. The extraction is correct when the neurons are attached to others, or when they cross each other. 


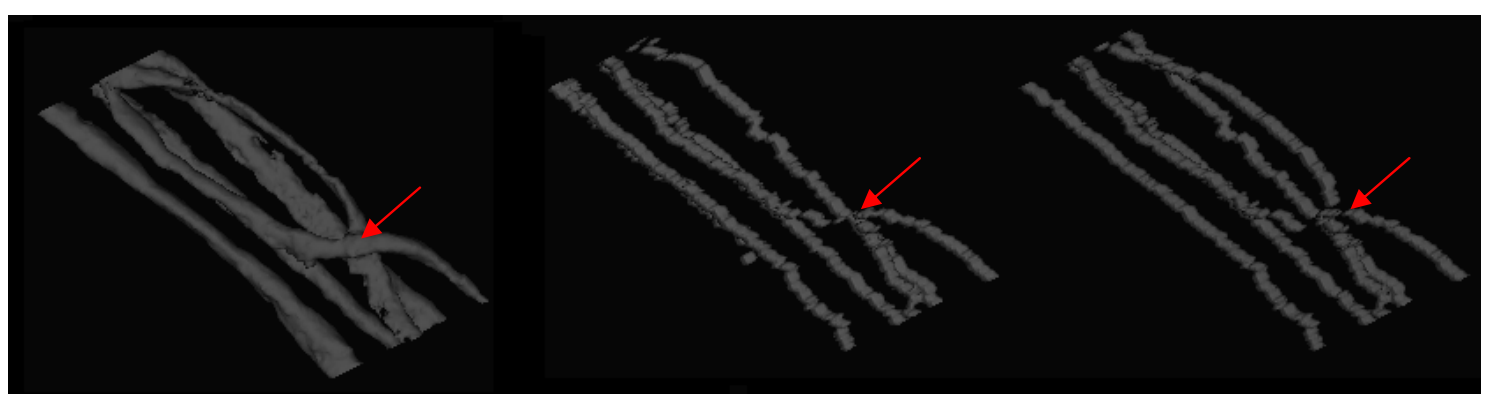

(a)

(b)

(c)

Figure 40. Partial 3D centerline extraction result to show the power of marker-controlled watershed segmentation. (a) The surface rendering of the original 3D neuron image stack. (b) The extracted 3D centerlines without marker-controlled watershed segmentation; (c) The extracted 3D centerlines with marker-controlled watershed segmentation; Notice that the neurons are attached at the location indicated by the red arrow. Clearly, the extraction is incorrect without the marker-controlled watershed segmentation.

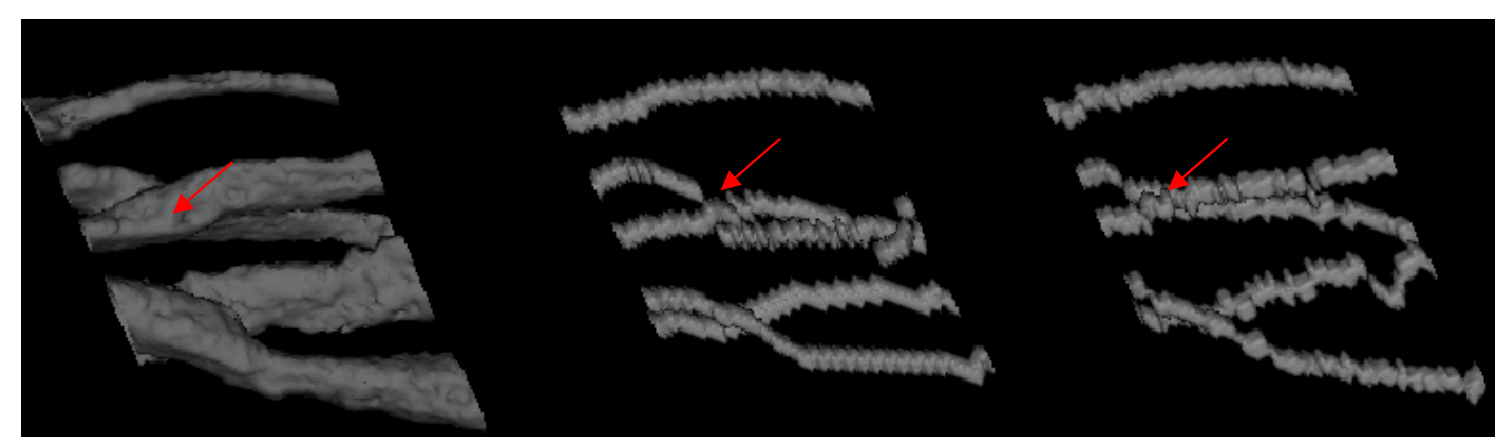

(a)

(b)

(c)

Figure 41. Partial 3D centerline extraction result to show the power of marker-controlled watershed segmentation. (a) The surface rendering of the original 3D neuron image stack. (b) The extracted 3D centerlines without marker-controlled watershed segmentation; (c) The extracted 3D centerlines with marker-controlled watershed segmentation; Notice that the neurons are attached at the location indicated by the red arrow. Clearly, the extraction is incorrect without the marker-controlled watershed segmentation.

\subsection{Conclusion and Discussion}

We presented a novel algorithm for extracting the centerlines of the neurons in 3D space. The algorithm is able to automatically and accurately track multiple $3 \mathrm{D}$ neurons in the image stack using 3D curvilinear structure detection. Our proposed method is able to extract centerline and boundaries of 3D neurite structures in 3D microscopy neuron images. The extraction is highly automated, rapid, and accurate, making it suitable for replacing the fully manual methods of extracting curvilinear structures when studying the $3 \mathrm{D}$ neuron images. 
The proposed method can handle complicated neuron structures such as cross-over sections and attaching segments.

The future work will focus on extracting more complicated neuron structures in the $3 \mathrm{D}$ space. An example of such more complicated cases is shown in Figure 42. Some neuron structures go through the same slice twice and may change the directions very sharply. We will study a sophisticated model for extracting such neuron structures. 

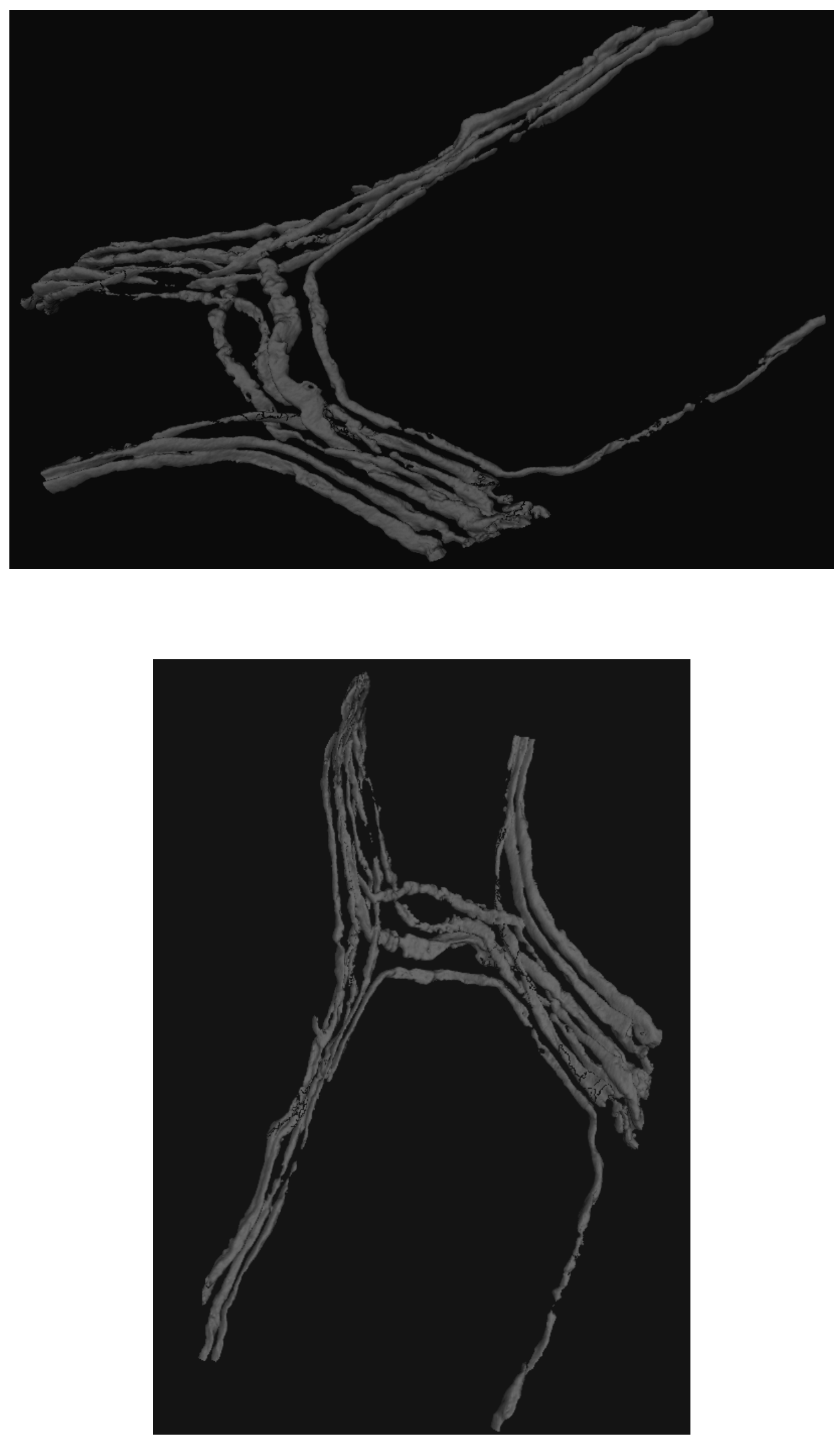

Figure 42. Two views of a 3D neuron image with very complicated neuron structures. In this case, one neuron may go across the same slice twice and may change directions very sharply. 


\section{Chapter 6: Conclusion and Discussion}

In this dissertation, we study two fundamental problems related to neuron images: lossless compression and neuron structure extraction. For lossless compression of neuron images, we start from studying the lossless compression of natural images. We have proposed PPAM - prediction by partial approximate matching for context modeling in lossless image compression. Rather than using exact contexts, PPAM models the contexts in an image in an approximate manner, based on $k$-approximate matching. Algorithms are proposed for efficient searching for approximate contexts using the PPAM context tree. To further reduce computational costs, the potentially huge contexts are clustered off-line, such that the required conditional probabilities can be determined at compression time using a simple lookup table. The input natural images are clustered based on their entropy and standard deviation after the prediction. Images with similar entropy and standard deviation are grouped together and only one image in that group is used for the time-consuming offline training. Once the context modeling is trained for the representative image, it can be used to compress other images in the group. Comparative results show that PPAM outperforms the original PPM method in modeling the contexts for natural images. The PPAM lossless image compression algorithm produced competitive performance in context modeling when compared with other state-of-the-art lossless image coding algorithms. The average coding performance of PPAM is better than that of EDP and JPEG-LS. The performance of PPAM can be further improved by developing an improved context quantization algorithm and by using more sophisticated image clustering techniques. The PPAM scheme can be easily extended to the lossless compression of neuron images. The neuron images are common in that they contain mostly the neuron structures. Thus all the neuron images acquired from the same experiment can be regarded as one group and only one neuron image is required for the training. The experiment 
shows that the PPAM outperforms most of the other state-of-the-art lossless compression schemes available for comparison when compressing neuron images. The PPAM scheme can also be applied to other types of images that contain common features, for example, microarray images, medical images (CT, MRI), and aerial images.

For neuron structure extraction, we have studied neuron centerline extraction in $2 \mathrm{D} / 3 \mathrm{D}$ spaces and dendritic spine extraction in 2D space. For 2D neuron centerline extraction, we present a novel algorithm specifically designed for high-throughput screening of neuronbased assays, which usually requires the process to be fast and automatic. The algorithm is able to automatically select a pair of starting point and ending point for each neurite segment so that only minimum user interaction is required. The starting point is selected by detecting the local maxima after low-pass filtering a grid of lines in the image, followed by signal-tonoise ratio analysis to filter robust points. The automatic selection of the ending point is achieved by iteratively tracing the points on or near the centerline of the neurite segment until the end of the neurite structure. Robust starting points and redundant ending points are used so that the extraction is complete. Dynamic programming is used to link the points so that the centerline can be extracted rapidly, accurately, and smoothly.

For the $2 \mathrm{D}$ dendritic spine extraction, we have studied the curvilinear structure detection based method. A novel boundary detection method is derived and applied to rapidly segment the backbone and spine areas. The algorithm can detect and quantitate both attached spines and detached spines. For attached spines, a classifier is used to further remove protrusions based on their geometry and moment features. At present, the algorithm is applied on the 2D projection of the 3D neuron image stacks. So, some of the 3D morphological characters, such as spine volume and spine shapes, are not available. In order to access the volumetric measurements, it is necessary to study the $3 \mathrm{D}$ dendritic structures, which can be achieved by extending the curvilinear structure detection method to 3D. The classification scheme used in 
this work is simple and effective, and it only can determine if an attached spine is valid or invalid. To study the functional significance of the dendritic spines, spines can be further classified as stubby, mushroom, and thin types, based on their shape characteristics. Such classification, however, requires more spine features and more sophisticated classification schemes.

For 3D neuron centerline extraction, we present a novel algorithm based on 3D dynamic programming and marker-controlled watershed segmentation. The algorithm is able to automatically and accurately track multiple $3 \mathrm{D}$ axons in the image stack. The extraction is highly automated, rapid, and accurate, making it suitable for replacing the fully manual methods of extracting curvilinear structures when studying the $3 \mathrm{D}$ axon images. The proposed method can handle complicated axon structures such as cross-over sections and attaching segments. The future work will focus on extracting more complicated neuron structures in the 3D space. Some neuron structures go through the same slice twice and may change directions very sharply. We will study sophisticated models for extracting such neuron structures. 


\section{References}

[1] A. Can, H. Shen, J. N. Turner, H. L. Tanenbaum, and B. Roysam, "Rapid automated tracing and feature extraction from live high-resolution retinal fundus images using direct exploratory algorithms," IEEE Transactions on Information Technology in Biomedicine, vol. 3, pp. 125-138, 1999.

[2] C. Steger, "An unbiased detector of curvilinear structure," IEEE Transactions on Pattern Analysis and Machine Intelligence, vol. 20, pp. 113-125, 1998.

[3] E. Meijering, M. Jacob, J.-C. F. Sarria, P. Steiner, H. Hirling, and M. Unser, "Design and validation of a tool for neurite tracing and analysis in fluorescence microscopy images," Cytometry, vol. 58A, pp. 167-176, 2004.

[4] A. Lempel and J. Ziv, "Compression of two-dimensional data," IEEE Transactions on Information Theory, vol. 32, pp. 2-8, 1986.

[5] X. Wu and N. Memon, "Context-based, adaptive, lossless image coding," IEEE Transactions on Communications, vol. 45, pp. 437-444, 1997.

[6] N. Memon and X. Wu, "Recent developments in context-based predictive techniques for lossless image compression," The Computer Journal, vol. 40, pp. 127-136, 1997.

[7] P. E. Tischer, R. T. Worley, A. J. Maeder, and M. Goodwin, "Context based lossless image compression," The Computer Journal, vol. 36, pp. 68-77, 1993.

[8] P. G. Howard and J. S. Vitter, "Fast and efficient lossless image compression," in Data Compression Conference, Snowbird, Utah, 1993, pp. 351-360.

[9] P. G. Howard, "The design and analysis of efficient lossless data compression systems," in Department of Computer Science. vol. Ph.D: Brown University, 1993.

[10] A. Moffat, "Two-level context based compression of binary images," in Data Compression Conference, Snowbird, Utah, 1991, pp. 328-391. 
[11] M. J. Weinberger, G. Seroussi, and G. Sapiro, "The LOCO-I lossless image compression algorithm: principles and standardization into JPEG-LS," IEEE Transactions on Image Processing, vol. 9, pp. 1309-1324, 2000.

[12] B. Meyer and P. Tischer, "Glicbawls - grey level image compression by adaptive weighted least squares," in Data Compression Conference, Snowbird, Utah, 2001.

[13] B. Meyer and P. Tischer, "Extending TMW for near lossless compression of greyscale images," in Data Compression Conference, Snowbird, Utah, 1998, pp. 458-470.

[14] J. G. Cleary and W. J. Teahun, "Unbounded length contexts for PPM," The Computer Journal, vol. 40, pp. 67-75, 1997.

[15] A. Moffat, "Implementing the PPM data compression scheme," IEEE Transactions on Communications, vol. 38, pp. 1917-1921, 1990.

[16] J. G. Cleary and I. H. Witten, "Data compression using adaptive coding and partial string matching," IEEE Transactions on Communications, vol. 32, pp. 396-402, 1984.

[17] I. H. Witten and T. C. Bell, "The zero-frequency problem: Estimating the probabilities of novel events in adaptive text compression," IEEE Transactions on Information Theory, vol. 37, pp. 1085-1094, 1991.

[18] S. Forchhammer and J. M. 1. Salinas, "Progressive coding of palette images and digital maps," in Data Compression Conference, Snowbird, Utah, 2002, pp. 362-371.

[19] P. Kopylov and P. Fränti, "Context tree compression of multi-component map images," in Data Compression Conference, Snowbird, Utah, 2002, pp. 212-221.

[20] M. Alzina, W. Szpankowski, and A. Grama, "2D-Pattern matching image and video compression: theory, algorithms, and experiments," IEEE Transactions on Image Processing, vol. 11, pp. 318-331, 2002. 
[21] A. A. Kassim, P. Yan, W. S. Lee, and K. Sengupta, "Motion compensated lossy-tolossless compression of 4-D medical images using integer wavelet transforms," IEEE Transactions on Information Technology in Biomedicine, vol. 9, pp. 132-138, 2005.

[22] Z. Xiong, X. Wu, S. Cheng, and J. Hua, "Lossy-to-lossless compression of medical volumetric data using three-dimensional integer wavelet transforms," IEEE Transactions on Medical Imaging, vol. 22, pp. 459-470, 2003.

[23] T. Bernas, E. K. Asem, J. P. Robinson, and B. Rajwa, "Compression of fluorescence microscopy images based on the signal-to-noise estimation," Microscopy Research and Technique, vol. 69, pp. 1-9, 2006.

[24] G. Navarro, "A guided tour of approximate string matching," ACM Computing Surveys, vol. 33, pp. 1-68, 1999.

[25] D. Gusfield, Algorithms on Strings, Trees, and Sequences: Computer Science and Computational Biology. Cambridge, UK: Cambridge University Press, 1997.

[26] D. E. Knuth, The art of computer programming: sorting and searching. Reading, MA: Addison-Wesley, 1973.

[27] J. Rissanen, "Universal coding, information, prediction and estimation," IEEE Transactions on Information Theory, vol. 30, pp. 629-636, 1984.

[28] J. Chen, "Context modeling based on context quantization with application in wavelet image coding," IEEE Transactions on Image Processing, vol. 13, 2004.

[29] S. Forchhammer, X. Wu, and J. D. Andersen, "Optimal context quantization in lossless compression of image data sequences," IEEE Transactions on Image Processing, vol. 13, pp. 509-517, 2004.

[30] E. Gokcay and Jose C. Principe, "Information theoretic clustering," IEEE Transactions on Pattern Analysis and Machine Intelligence, vol. 24, pp. 158-171, 2002. 
[31] T. M. Cover and J. A. Thomas, Elements of Information Theory: Wiley-Interscience, 1991.

[32] X. Zhou and T. C. W. Stephen, "Informatics challenges of high-throughput cellular and molecular microscopy," IEEE Signal and Processing Magazine, vol. 23, pp. 63$72,2006$.

[33] X. Li and M. T. Orchard, "Edge-directed prediction for lossless compression of natural images," IEEE Transactions on Image Processing, vol. 10, pp. 813-817, 2001.

[34] Y. Feng, "Practicing cell morphology based screen," European Pharmaceutical Review, vol. 7, pp. 7-11, 2002.

[35] A. Cacace, M. Banks, T. Spicerb, F. Civolic, and J. Watson, "An ultra-HTS process for the identification of small molecule modulators of orphan G-protein-coupled receptors," Drug Discovery Today, vol. 8, pp. 785-792, 2003.

[36] R. Dunkle, "Role of Image informatics in accelerating drug discovery and development," Drug Discovery World, vol. 5, pp. 75-82, 2003.

[37] S. J. Fox, "Accommodating cells in HTS," Drug Discovery World, vol. 5, pp. 21-30, 2003.

[38] F. Gaunitz and K. Heise, "HTS compatible assay for antioxidative agents using primary cultured hepatocytes," ASSAY and Drug Development Technologies, vol. 1, pp. 469-477, 2003.

[39] V. Klyushnichenko, "Protein crystallization: from HTS to kilogram-scale," Current Opinion in Drug Discovery \& Development, vol. 6, pp. 848-854, 2003.

[40] J. C. Yarrow, Y. Feng, Z. E. Perlman, T. Kirchhausen, and T. J. Mitchison, "Phenotypic screening of small molecule libraries by high throughput cell imaging," Combinatorial Chemistry \& High Throughput Screening, vol. 6, pp. 279-286, 2003. 
[41] Z. E. Perlman, M. D. Slack, Y. Feng, T. J. Mitchison, L. F. Wu, and S. J. Altschuler, "Multidimensional drug profiling by automated microscopy," Science, vol. 306, pp. 1194-1198, 2004.

[42] K. A. Al-Kofahi, A. Can, S. Lasek, D. H. Szarowski, N. Dowell-Mesfin, W. Shain, J. N. Turner, and B. Roysam, "Median-based robust algorithms for tracing neurons from noisy confocal microscope images," IEEE Transactions on Information Technology In Biomedicine, vol. 7, pp. 302-317, 2003.

[43] K. A. Al-Kofahi, S. Lasek, D. H. Szarowski, C. J. Pace, G. Nagy, J. N. Turner, and B. Roysam, "Rapid automated three-dimensional tracing of neurons from confocal image stacks," IEEE Transactions on Information Technology in Biomedicine, vol. 6, pp. 171-187, 2002.

[44] R. Polli and G. Valli, "An algorithm for real-time vessel enhancement and detection," Comput Methods Programs Biomed, vol. 52, pp. 1-22, 1997.

[45] R. D. T. Janssen and A. M. Vossepoel, "Adaptive vectorization of line drawing images," Computer vision and image understanding, vol. 65, pp. 38-56, 1997.

[46] J. L. Coatrieux, M. Garreau, R. Collorec, and C. Roux, "Computer vision approaches for the three-dimensional reconstruction: Review and prospects," Critical Reviews in Biomedical Engineering, vol. 22, pp. 1-38, 1994.

[47] R. Collorec and J. L. Coatrieux, "Vectorial tracking and directed contour finder for vascular network in digital subtraction angiography," Pattern Recognition Letters, vol. 8, pp. 353-358, 1988.

[48] O. Wink, A. F. Frangi, B. Verdonck, M. A. Viergever, and W. J. Niessen, "3D MRA coronary axis determination using a minimum cost path approach," Magnetic Resonance in Medicine, vol. 47, pp. 1169-1175, 2002. 
[49] A. F. Frangi, W. J. Niessen, R. M. Hoogeveen, T. v. Walsum, and M. A. Viergever, "Model-based quantitation of 3-D magnetic resonance angiographic images," IEEE Transactions on Medical Imaging, vol. 18, pp. 946-956, 1999.

[50] Y. Sato, S. Nakajima, N. Shiraga, H. Atsumi, S. Yoshida, T. Koller, G. Gerig, and R. Kikinis, "Three-dimensional multi-scale line filter for segmentation and visualization of curvilinear structures in medical images," Medical Image Analysis, vol. 2, pp. 143$168,1998$.

[51] C. Steger, "Extracting curvilinear structures: A differential geometric approach," in 4th European Conference on Computer Vision, Cambridge, UK, 1996, pp. 630-641.

[52] J. B. A. Maintz, P. A. v. d. Elsen, and M. A. Viergever, "Evaluation of ridge seeking operators for multimodality medical image matching," IEEE Transactions on Pattern Analysis and Machine Intelligence, vol. 18, pp. 353-365, 1996.

[53] I. S. Kweon and T. Kanade, "Extracting topographic terrain features from elevation maps," Computer Vision, Graphics, and Image Processing: Image Understanding, vol. 59, pp. 171-182, 1994.

[54] L. Wang and T. Pavlidis, "Direct gray-scale extraction of features for character recognition," IEEE Transactions on Pattern Analysis and Machine Intelligence, vol. 15, pp. 1053-1067, 1993.

[55] L. Wang and T. Pavlidis, "Detection of curved and straight segments from gray scale topography," Computer Vision, Graphics, and Image Processing: Image Understanding, vol. 58, pp. 352-365, 1993.

[56] S. Chaudhuri, S. Chatterjee, N. Katz, M. Nelson, and M. Goldbaum, "Detection of blood vessels in retinal images using two-dimensional matched filters," IEEE Transactions on Medical Imaging, vol. 3, pp. 263-269, 1989. 
[57] T. Lindeberg, "Edge detection and ridge detection with automatic scale selection," International Journal of Computer Vision, vol. 30, pp. 117-154, 1998.

[58] L. A. Iverson and S. W. Zucker, "Logical/Linear operators for image curves," IEEE Transactions on Pattern Analysis and Machine Intelligence, vol. 17, pp. 982-996, 1995.

[59] L. M. J. Florack, B. M. t. H. Romeny, J. J. Koenderink, and M. A. Viergever, "Scale and the differential structure of images," Image and Vision Computing, vol. 10, pp. 376-388, 1992.

[60] A. X. Falc ao, J. K. Udupa, and F. K. Miyazawa, "An ultra-fast user-steered image segmentation paradigm: Livewire on the fly," IEEE Transactions on Medical Imaging, vol. 19, pp. 55-62, 2000.

[61] E. N. Mortensen and W. A. Barrett, "Interactive segmentation with intelligent scissors," Graphical Models and Image Processing, vol. 60, pp. 349-384, 1998.

[62] A. X. Falc ao, J. K. Udupa, S. Samarasekera, and S. Sharma, "User-steered image segmentation paradigms: Livewire and live lane," Graphical Models and Image Processing, vol. 60, pp. 233-260, 1998.

[63] W. A. Barrett and E. N. Mortensen, "Interactive live-wire boundary extraction," Medical Image Analysis, vol. 1, pp. 331-341, 1996.

[64] N. Otsu, "A threshold selection method from gray-level histograms," IEEE Transactions on Systems, Man, and Cybernetics, vol. 9, pp. 62-66, 1979.

[65] Y. Sun, R. J. Lucariello, and S. A. Chiaramida, "Directional lowpass filtering for improved accuracy and reproducibility of stenosis quantification in coronary arteriograms," IEEE Transactions on Medical Imaging, vol. 14, pp. 242-248, 1995.

[66] Y. Zhang, X. Zhou, A. Degterev, M. Lipinski, D. Adjeroh, J. Yuan, and S. T. C. Wong, "A novel tracing algorithm for high throughput imaging screening of neuron 
based assays," Journal of Neuroscience Methods, accepted for publication, vol. accepted for publication, 2006.

[67] R. E. Bellman and S. E. Dreyfus, Applied dynamic programming: Princeton University Press, 1962.

[68] T. H. Cormen, C. E. Leiserson, R. L. Rivest, and C. Stein, Introduction to algorithms, 2nd ed.: The MIT Press, 2001.

[69] A. A. Amini, T. E. Weymouth, and R. C. Jain, "Using dynamic programming for solving variational problems in vision," IEEE Transactions Pattern Analysis and Machine Intelligence, vol. 12, pp. 855-867, 1990.

[70] H. Yamada, C. Merritt, and T. Kasvand, "Recognition of kidney glomerulus by dynamic programming matching method," IEEE Transactions Pattern Analysis and Machine Intelligence, vol. 10, pp. 731-737, 1988.

[71] D. Geiger, A. Gupta, L. A. Costa, and J. Vlontzos, "Dynamic programming for detecting, tracking, and matching deformable contours," IEEE Transactions on Pattern Analysis and Machine Intelligence, vol. 17, pp. 294-302, 1995.

[72] E. Mortensen, B. Morse, W. Barrett, and J. Udupa, "Adaptive boundary detection using 'live-wire' two-dimensional dynamic programming," in Computers in Cardiology 1992. Proceedings, Durham, NC, 1992, pp. 635 - 638.

[73] E. W. Dijkstra, "A note on two problems in connexion with graphs," Numerische Mathematik, vol. 1, pp. 269-271, 1959.

[74] J. W. Lydon, "The measurement of the modal mineralogy of rocks from SEM imagery: the use of Multispec $(\mathbb{C}$ and ImageJ freeware," in Geological Survey of Canada, Open File 4941: GEOSCAN, 2005.

[75] E. A. Nimchinsky, B. L. Sabatini, and K. Svoboda, "Structure and function of dendritic spines," Annual review of physiology, vol. 64, pp. 313-353, 2002. 
[76] S. F. Tavazoie, V. A. Alvarez, D. A. Ridenour, D. J. Kwiatkowski, and B. L. Sabatini, "Regulation of neuronal morphology and function by the tumor suppressors Tsc1 and Tsc2," Nature Neuroscience, vol. 8, pp. 1727-1734, 2005.

[77] D. D. Sarbassov, S. M. Ali, and D. M. Sabatini, "Growing roles for the mTOR pathway," Current Opinion in Cell Biology, vol. 17, pp. 596-603, 2005.

[78] A. G. Carter and B. L. Sabatini, "State-dependent calcium signaling in dendritic spines of striatal medium spiny neurons," Neuron, vol. 44, pp. 483-493, 2004.

[79] X. Zhou, K. Liu, B. L. Sabatini, and S. T. C. Wong, "Mutual information based feature selection in studying perturbation of dendritic structure caused by TSC2 inactivation," Neuroinformatics, vol. 4, pp. 81-94, 2006.

[80] B. L. Sabatini, M. Maravall, and K. Svoboda, "Ca(2+) signaling in dendritic spines," Current Opinion in Cell Biology, vol. 11, pp. 349-356, 2001.

[81] B. Lendvai, E. A. Stern, B. Chen, and K. Svoboda, "Experience-dependent plasticity of dendritic spines in the developing rat barrel cortex in vivo," Nature, vol. 404, pp. 876-881, 2000.

[82] M. Maletic-Savatic, R. Malinow, and K. Svoboda, "Rapid dendritic morphogenesis in CA1 hippocampal dendrites induced by synaptic activity," Science, vol. 283, pp. 1923-1927, 1999.

[83] F. Engert and T. Bonhoeffer, "Dendritic spine changes associated with hippocampal long-term synaptic plasticity," Nature, vol. 399, pp. 66-70, 1999.

[84] A. Herzog, G. Krell, B. Michaelis, J. Wang, W. Zuschratter, and A. K. Braun, "Restoration of three-dimensional quasi-binary images from confocal microscopy and its application to dendritic trees," in Proc. SPIE Vol. 2984, p. 146-157, ThreeDimensional Microscopy: Image Acquisition and Processing IV Bellingham, WA, 1997. 
[85] I. Y. Y. Koh, W. B. Lindquist, K. Zito, E. A. Nimchinsky, and K. Svoboda, "An image analysis algorithm for dendritic spines," Neural Computation, vol. 14, pp. 1283-1310, 2002.

[86] C. M. Weaver, P. R. Hof, S. L. Wearne, and W. B. Lindquist, "Automated algorithms for multiscale morphometry of neuronal dendrites," Neural Computation, vol. 16, pp. 1353-1383, 2004.

[87] G. M. P. v. Kempen, L. J. v. Vliet, P. J. Verveer, and H. T. M. V. d. Voort, "A quantitative comparison of image restoration methods for confocal microscopy," Journal of Microscopy, vol. 185, pp. 354-365, 1996.

[88] J. S. Lim, Two-dimensional Signal and Image Processing: Prentice Hall PTR, 1989.

[89] G. Xiong, X. Zhou, A. Degterev, L. Ji, and S. T. C. Wong, "Automated neurite labeling and analysis in fluorescence microscopy images," Cytometry, vol. 69A, pp. 494-505, 2006.

[90] J. E. Bresenham, "Algorithm for computer control of a digital plotter," IBM Systems Journal, vol. 4, pp. 25-30, 1965.

[91] K. M. Harris, "Structure, development, and plasticity of dendritic spines," Current Opinion Neurobiology, vol. 9, pp. 343-348, 1999.

[92] A. Khotanzad and Y. H. Hong, "Invariant image recognition by Zernike moments," IEEE Transactions on Pattern Analysis and Machine Intelligence, vol. 12, pp. 489497, 1990.

[93] C.-H. Teh and R. T. Chin, "On image analysis by the methods of moments," IEEE Transactions Pattern Analysis and Machine Intelligence, vol. 10, pp. 496-513, 1988.

[94] E. Cantu-Paz, "Feature subset selection by estimation of distribution algorithms," in Genetic and Evolutionary Computation Conference (GECCO) San Francisco, CA, 2002. 
[95] J. H. Holland, Adaptation in Natural and Artificial Systems: An Introductory Analysis with Applications to Biology, Control and Artificial Intelligence, 2 ed. Cambridge, MA: MIT Press, 1996.

[96] P. Pudil, F. J. Ferri, J. Novovicova, and J. Kittler, "Floating search methods for feature selection with nonmonotonic criterion functions," in Proceedings of the 12th IAPR International. Conference on Pattern Recognition - Conference B: Computer Vision \& Image Processing., , 1994.

[97] F. J. Ferri, P. Pudil, M. Hatef, and J. Kittler, "Comparative study of techniques for large-scale feature selection," Pattern Recognition in Practice IV, pp. 403-413, 1994.

[98] A. Jain and D. Zongker, "Feature selection: evaluation, application, and small sample performance," IEEE Transactions on Pattern Analysis and Machine Intelligence, vol. 19, pp. 153-158, 1997.

[99] K. Fukunaga, Introduction to Statistical Pattern Recognition. San Diego, California: Academic Press, 1990.

[100] C. R. Keller-Peck, M. K. Walsh, W.-B. Gan, G. Feng, J. R. Sanes, and J. W. Lichtman, "Asynchronous synapse elimination in neonatal motor units: studies using GFP transgenic mice," Neuron, vol. 31, pp. 381-394, 2001.

[101] H. Kasthuri and J. W. Lichtman, "The role of neuronal identity in synaptic competition," Nature, vol. 424, pp. 426-430, 2003.

[102] P. D. Coleman, C. F. Garvey, J. Young, and W. Simon, Semiautomatic tracing of neuronal processes: Plenum Press, 1977.

[103] C. F. Garvey, J. Young, W. Simon, and P. D. Coleman, "Automated threedimensional dendrite tracking system," Electroencephalogr Clin Neurophysiol, vol. 35, pp. 199-204, 1973. 
[104] J. J. Capowski, Computer techniques in neuroanatomy: New York : Plenum Press, 1989.

[105] A. R. Cohen, B. Roysam, and J. N. Turner, "Automated tracing and volume measurements of neurons from 3-D confocal fluorescence microscopy data," Journal of microscopy, vol. 173, pp. 103-114, 1994.

[106] W. He, T. A. Hamilton, A. R. Cohen, T. J. Holmes, C. Pace, D. H. Szarowski, J. N. Turner, and B. Roysam, "Automated three-dimensional tracing of neurons in confocal and brightfield images," Microscopy and microanalysis, vol. 9, pp. 296-310, 2003.

[107] G. Lin, C. S. Bjornsson, K. L. Smith, A. M. Abdul-Karim, J. N. Turner, W. Shain, and B. Roysam, "Automated image analysis methods for 3-D quantification of the neurovascular unit from multichannel confocal microscope images," Cytometry Part A, vol. 66A, pp. 9-23, 2005.

[108] H. Shen, B. Roysam, C. V. Stewart, J. N. Turner, and H. L. Tanenbaum, "Optimal scheduling of tracing computations for real-time vascular landmark extraction from retinal fundus images," IEEE Transactions on Information Technology in Biomedicine, vol. 5, pp. 77-91, 2001.

[109] F. Xu, P. H. Lewis, J. E. Chad, and H. V. Wheal, "Extracting generalized cylinder models of dendritic trees from 3D image stacks," in Three-Dimensional and Multidimensional Microscopy: Image Acquisition and Processing V, 1998, pp. 149158.

[110] A. Herzog, G. Krell, B. Michaelis, and W. Zuschratter, "Tracking on tree-like structures in 3-D confocal images," in Three-Dimensional and Multidimensional Microscopy: Image Acquisition and Processing V, 1998, pp. 165-176.

[111] J. C. Fiala, "Reconstruct Software: http://synapses.bu.edu/tools/index.htm," 2006.

[112] Bitplane, "Imaris Software: http://www.bitplane.com/products/index.shtml," 2006. 
[113] F. Meyer, "Iterative image transformations for an automatic screening of cervical cancer," Journal of Histochemistry and Cytochemistry, vol. 27, pp. 128-135, 1979.

[114] R. Adams, "Radial decomposition of discs and spheres," Computer Vision, Graphics, and Image Processing: Graphical Models and Image Processing, vol. 55, pp. 325$332,1993$.

[115] R. C. Gonzalez and R. E. Woods, Digital Image Processing. Upper Saddle River, NJ: Prentice-Hall, 2002. 